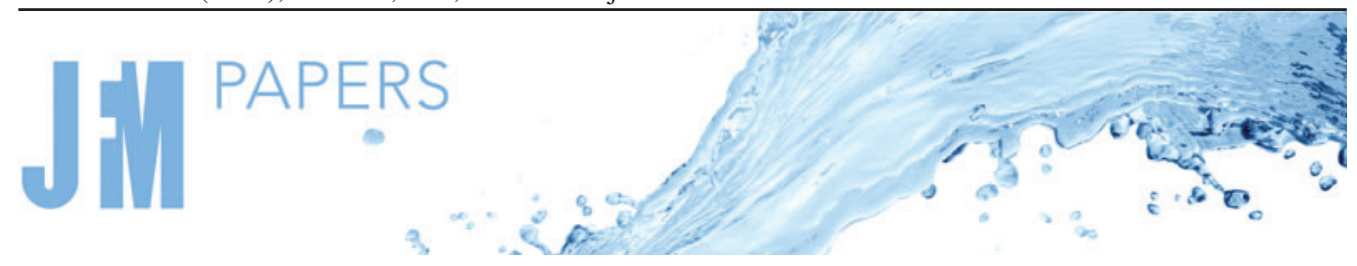

\title{
Inversion of the transverse force on a spinning sphere moving in a rarefied gas
}

\author{
Satoshi Taguchi ${ }^{1,2}+$ and Tetsuro Tsuji ${ }^{1,2}$ \\ ${ }^{1}$ Department of Advanced Mathematical Sciences, Graduate School of Informatics, Kyoto University, \\ Kyoto 606-8501, Japan \\ ${ }^{2}$ Research Project of Fluid Science and Engineering, Advanced Engineering Research Center, \\ Kyoto University, Japan
}

(Received 25 May 2021; revised 7 October 2021; accepted 21 November 2021)

The flow around a spinning sphere moving in a rarefied gas is considered in the following situation: (i) the translational velocity of the sphere is small (i.e. the Mach number is small); (ii) the Knudsen number, the ratio of the molecular mean free path to the sphere radius, is of the order of unity (the case with small Knudsen numbers is also discussed); and (iii) the ratio between the equatorial surface velocity and the translational velocity of the sphere is of the order of unity. The behaviour of the gas, particularly the transverse force acting on the sphere, is investigated through an asymptotic analysis of the Boltzmann equation for small Mach numbers. It is shown that the transverse force is expressed as $\boldsymbol{F}_{L}=\pi \rho a^{3}(\boldsymbol{\Omega} \times \boldsymbol{v}) \bar{h}_{L}$, where $\rho$ is the density of the surrounding gas, $a$ is the radius of the sphere, $\boldsymbol{\Omega}$ is its angular velocity, $\boldsymbol{v}$ is its velocity and $\bar{h}_{L}$ is a numerical factor that depends on the Knudsen number. Then, $\bar{h}_{L}$ is obtained numerically based on the Bhatnagar-Gross-Krook model of the Boltzmann equation for a wide range of Knudsen number. It is shown that $\bar{h}_{L}$ varies with the Knudsen number monotonically from 1 (the continuum limit) to $-\frac{2}{3}$ (the free molecular limit), vanishing at an intermediate Knudsen number. The present analysis is intended to clarify the transition of the transverse force, which is previously known to have different signs in the continuum and the free molecular limits.

Key words: non-continuum effects, kinetic theory

\section{Introduction}

The flow around a very small spherical particle moving in a gas is fundamental in fluid mechanics and plays a vital role in many applications such as aerosol transport and particle manipulations in nano- and micro-technologies. When the particle size is comparable

$†$ Email address for correspondence: taguchi.satoshi.5a@kyoto-u.ac.jp 


\section{S. Taguchi and T. Tsuji}

with the mean free path of the gas molecules, conventional fluid mechanics is no longer applicable. Instead, kinetic theory provides a suitable framework for investigating the flow around such a very small particle. This paper investigates forces acting on a sphere moving in a rarefied gas based on kinetic theory.

Let us restrict our consideration to the case where the Mach number based on the particle velocity relative to the surrounding gas is small. The sphere may be rotating around one of its axes. Moreover, the sphere's circumferential velocity is supposed to be of same order of magnitude as the sphere's translational speed. It, therefore, is small compared to the sound speed (or the thermal speed of the gas molecules). The drag (e.g. Knudsen \& Weber 1911; Epstein 1924; Willis 1966; Cercignani, Pagani \& Bassanini 1968; Sone \& Aoki 1977a,b; Law \& Loyalka 1986; Aoki \& Sone 1987; Beresnev, Chernyak \& Fomyagin 1990; Loyalka 1992; Takata, Sone \& Aoki 1993; Kalempa \& Sharipov 2020) and torque (e.g. Loyalka 1992; Andreev \& Popov 2010; Taguchi, Saito \& Takata 2019) acting on the sphere in this situation have been investigated extensively in the past. However, the understanding of the transverse force (i.e. lift force), resulting from the interplay between the translational and swirling motion of the gas around the sphere, is still unsatisfactory, as described below.

The transverse force acting on a rotating sphere translating in a highly rarefied gas was investigated by, for example, Wang (1972), Ivanov \& Yanshin (1980), Borg, Söderholm \& Essén (2003) and Liu \& Bogy (2008). In those studies, assuming a free molecular (or collisionless) gas, it was shown that the transverse force has the opposite sign as compared with the corresponding force in the continuum flow (Rubinow \& Keller 1961). Therefore, as pointed out in Borg et al. (2003), there will be a critical value of the Knudsen number at which the transverse force vanishes and above which the force reverses its direction. Here, the Knudsen number is defined as the reciprocal of the ratio of the sphere size to the molecular mean free path. Indeed, a gradual transition of the transverse force with the Knudsen number is observed in a numerical study using the direct simulation Monte Carlo (DSMC) method (Volkov 2011). However, the precise determination of the critical point remains an open question. In this study, we take a step further and clarify the transition of the transverse force between the two limits, namely the continuum and the free molecular limits.

As the basic equation, we employ the Boltzmann equation and, for simplicity, we assume the diffuse reflection boundary condition on the sphere. In the actual numerical computations, we use the Bhatnagar-Gross-Krook (BGK) model (Bhatnagar, Gross \& Krook 1954; Welander 1954) of the Boltzmann equation to simplify the problem further and make the numerical analysis tractable.

The paper is organized as follows. The problem is formulated and scaling assumptions are introduced in $\S 2$. In $\S 3$, we carry out an asymptotic analysis of the Boltzmann system for small Mach numbers and derive general expressions for the drag, lift and torque acting on the sphere. Section 4 is devoted to the actual computation of the transverse force. Section 5 presents concluding remarks.

\section{Formulation}

\subsection{Problem and basic assumptions}

Let us consider a rigid sphere with radius $L$ moving through a monatomic ideal gas with constant translational velocity $\boldsymbol{v}_{0}$. While translating, the sphere is also rotating around an axis of revolution with a constant angular velocity, $\boldsymbol{\Omega}_{0}$. Far from the sphere, the gas is in the equilibrium state at rest with pressure $p_{0}$ and temperature $T_{0}$. Further, we assume that the sphere's surface temperature is uniform and is equal to the gas temperature at infinity. 


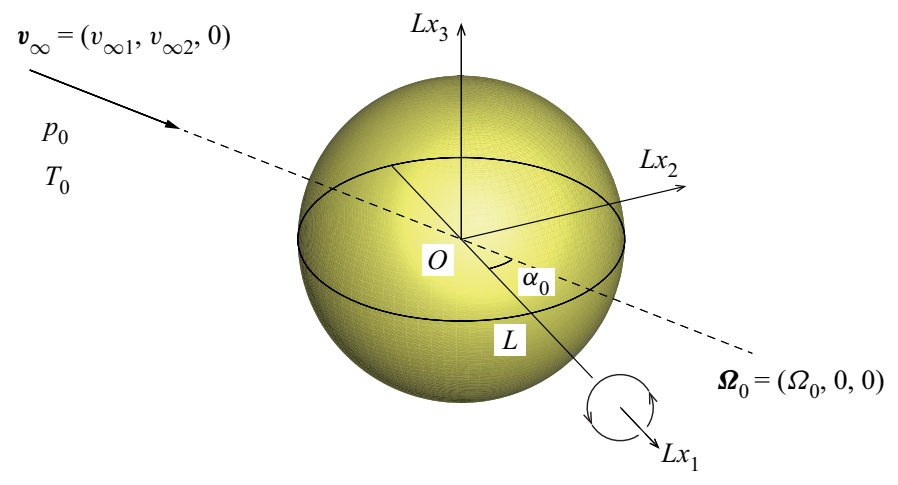

Figure 1. Problem.

For convenience, let us choose a frame of reference translating with the sphere. In this frame, the sphere centre is at rest, and the flow velocity at infinity is $-v_{0}$. We write this velocity as $\boldsymbol{v}_{\infty}\left(=-\boldsymbol{v}_{0}\right)$. We are now concerned with a steady flow of a rarefied gas past a rotating sphere, as shown in figure 1 . We investigate the behaviour of the gas under the following assumptions.

(i) The behaviour of the gas is described by the Boltzmann equation (we use the BGK model of the Boltzmann equation for the actual numerical computations).

(ii) The gas molecules undergo diffuse reflection on the sphere. More precisely, the velocity distribution of the reflected molecules on the surface constitutes the corresponding part of the Maxwellian distribution characterized by the temperature and (local) surface velocity of the sphere and by the condition that there is no net mass flux across the surface.

(iii) The translational speed of the sphere (or the flow speed at infinity in our frame) is small compared with the thermal speed of the gas molecules, i.e. $\left|\boldsymbol{v}_{\infty}\right| \ll\left(2 R T_{0}\right)^{1 / 2}$. Here, $R=k_{B} / m$ is the specific gas constant with $k_{B}$ and $m$ being the Boltzmann constant and the mass of a molecule, respectively. In other words, the Mach number of the flow, $M a=\left|v_{\infty}\right| /\left(5 R T_{0} / 3\right)^{1 / 2}$, is small.

(iv) The rotational surface velocity of the sphere is of the same order of magnitude as the translational velocity of the sphere, i.e. $L\left|\boldsymbol{\Omega}_{0}\right| /\left|\boldsymbol{v}_{\infty}\right|=O(1)$.

For the subsequent analysis, we introduce the rectangular coordinate system $L x_{i}(i=$ $1,2,3)$ with its origin at the centre of the sphere (the corresponding position vector is denoted by $L \boldsymbol{x}$ ). Without loss of generality, we can assume that the $x_{1}$ axis is parallel to the angular velocity $\boldsymbol{\Omega}_{0}$ and that the vector $\boldsymbol{v}_{\infty}$ lies in the $x_{1} x_{2}$ plane (see figure 1 ). Then, $\boldsymbol{\Omega}_{0}$ and $\boldsymbol{v}_{\infty}$ are expressed as $\boldsymbol{\Omega}_{0}=\left(\Omega_{0}, 0,0\right)$ and $\boldsymbol{v}_{\infty}=\left(v_{\infty 1}, v_{\infty 2}, 0\right)$ with $v_{\infty 1}, v_{\infty 2}$ and $\Omega_{0}\left(=\left|\boldsymbol{\Omega}_{0}\right|\right)$ being given constants.

\subsection{Basic equations}

Let us first introduce the following notation. The molecular velocity is denoted by $\xi_{i}$, $i=1,2,3$ (or by $\xi$ ) and the velocity distribution function by $f$. Furthermore, we denote by $\rho$ the density, by $v_{i}$ (or $\boldsymbol{v}$ ) the flow velocity, by $T$ the temperature, by $p$ the pressure, by $p_{i j}$ the stress tensor and by $q_{i}$ (or $q$ ) the heat-flux vector of the gas $(i, j=1,2,3$ ). Then, we 


\section{S. Taguchi and T. Tsuji}

introduce the following dimensionless variables:

$$
\begin{gathered}
\zeta_{i}=\xi_{i} /\left(2 R T_{0}\right)^{1 / 2}, \quad \phi(\boldsymbol{x}, \zeta)=E^{-1}\left[f / \rho_{0}\left(2 R T_{0}\right)^{-3 / 2}\right]-1, \\
\omega(\boldsymbol{x})=\rho / \rho_{0}-1, \quad u_{i}(\boldsymbol{x})=v_{i} /\left(2 R T_{0}\right)^{1 / 2}, \quad \tau(\boldsymbol{x})=T / T_{0}-1, \\
P(\boldsymbol{x})=p / p_{0}-1, \quad P_{i j}(\boldsymbol{x})=p_{i j} / p_{0}-\delta_{i j}, \quad Q_{i}(\boldsymbol{x})=q_{i} /\left[p_{0}\left(2 R T_{0}\right)^{1 / 2}\right],
\end{gathered}
$$

where $\rho_{0}=p_{0} /\left(R T_{0}\right), E=\pi^{-3 / 2} \exp \left(-|\zeta|^{2}\right)$ and $\delta_{i j}$ is the Kronecker delta.

We also use the spherical coordinate system $(\operatorname{Lr}, \theta, \varphi)$ related to $x_{i}$ by $x_{1}=r \cos \theta$, $x_{2}=r \sin \theta \cos \varphi$ and $x_{3}=r \sin \theta \sin \varphi$. Components of vectors and tensors in spherical coordinates are represented by $(r, \theta, \varphi)$ in the subscript, e.g. $\left(\zeta_{r}, \zeta_{\theta}, \zeta_{\varphi}\right), P_{r \theta}$, etc. Note that Cartesian components of a vector $a_{i}$ are related to $\left(a_{r}, a_{\theta}, a_{\varphi}\right)$ as

$$
\begin{gathered}
a_{1}=a_{r} \cos \theta-a_{\theta} \sin \theta, \\
a_{2}=a_{r} \sin \theta \cos \varphi+a_{\theta} \cos \theta \cos \varphi-a_{\varphi} \sin \varphi, \\
a_{3}=a_{r} \sin \theta \sin \varphi+a_{\theta} \cos \theta \sin \varphi+a_{\varphi} \cos \varphi .
\end{gathered}
$$

Throughout the paper, we write $\zeta$ to denote $|\zeta|=\left(\zeta_{j}^{2}\right)^{1 / 2}$.

The time-independent Boltzmann equation for $\phi$ is written as

$$
\zeta_{i} \frac{\partial \phi}{\partial x_{i}}=\frac{1}{k}(\mathscr{L}(\phi)+\mathscr{J}(\phi, \phi))
$$

where $\mathscr{L}$ and $\mathscr{J}$ represent, respectively, the linearized and nonlinear collision operators, whose explicit forms are given in Appendix A (see also Sone 2007). Parameter $k$ is defined by

$$
k=\frac{\sqrt{\pi}}{2} K n=\frac{\sqrt{\pi}}{2} \frac{\ell_{0}}{L},
$$

where $K n=\ell_{0} / L$ is the Knudsen number with $\ell_{0}$ being the mean free path of the gas molecules in the equilibrium state at rest with density $\rho_{0}$ and temperature $T_{0}$. For a hard-sphere gas, $\ell_{0}$ is given by $\ell_{0}=1 /\left[\sqrt{2} \pi d_{m}^{2}\left(\rho_{0} / m\right)\right]$ with $d_{m}$ the diameter of a molecule. On the other hand, for the BGK model introduced below, $\ell_{0}$ is given by $\ell_{0}=(2 / \sqrt{\pi})\left(2 R T_{0}\right)^{1 / 2} / A_{c} \rho_{0}$ with $A_{c}$ being a constant $\left(A_{c} \rho_{0}\right.$ is the collision frequency at the reference equilibrium state at rest). Further details concerning (2.3) are given in Appendix A.

The operators $\mathscr{L}$ and $\mathscr{J}$ are spherically symmetric operators, that is, for any functions $F$ and $G$ of $\zeta$, it holds that $\mathscr{L}\left(F\left(l_{i j} \zeta_{j}\right)\right)(\zeta)=\mathscr{L}(F(\zeta))\left(l_{i j} \zeta_{j}\right)$ and $\mathscr{J}\left(F\left(l_{i j} \zeta_{j}\right), G\left(l_{i j} \zeta_{j}\right)\right)(\zeta)=\mathscr{J}(F(\zeta), G(\zeta))\left(l_{i j} \zeta_{j}\right)$, where $l_{i j}$ is any orthogonal transformation, i.e. $l_{i j} l_{k j}=\delta_{i k}$. This implies that $\mathscr{L}$ and $\mathscr{J}$ are axially symmetric, meaning that they satisfy the same identities as above for any orthogonal transformation $l_{i j}$ that satisfies $l_{i j} a_{j}=a_{i}$, where $a_{i}$ is a fixed (unit) vector. Since $\mathscr{L}$ and $\mathscr{J}$ are spherically symmetric, $a_{i}$ can be chosen arbitrarily. The property of axial symmetry of the operators plays a crucial role in the present analysis.

The diffuse reflection boundary condition (e.g. Kogan 1969; Cercignani 1988; Sone 2007) on the sphere is written as

$$
\phi=\frac{1+\sigma_{w}}{\pi^{3 / 2}} \exp \left(-\zeta_{r}^{2}-\zeta_{\theta}^{2}-\left(\zeta_{\varphi}-\hat{\Omega}_{0} \sin \theta\right)^{2}\right) E^{-1}-1, \quad \zeta_{r}>0 \quad(r=|x|=1),
$$




\section{Inversion of the transverse force on a spinning sphere}

with

$$
\sigma_{w}=-2 \sqrt{\pi} \int_{\zeta_{r}<0} \zeta_{r} \phi E \mathrm{~d} \zeta
$$

where $\mathrm{d} \zeta=\mathrm{d} \zeta_{1} \mathrm{~d} \zeta_{2} \mathrm{~d} \zeta_{3}$ and $\hat{\Omega}_{0}$ is the dimensionless angular velocity defined by

$$
\hat{\Omega}_{0}=\frac{L \Omega_{0}}{\left(2 R T_{0}\right)^{1 / 2}} .
$$

The boundary condition at infinity is written as

$$
\phi \rightarrow \frac{1}{\pi^{3 / 2}} \exp \left(-\left(\zeta_{1}-\hat{v}_{\infty 1}\right)^{2}-\left(\zeta_{2}-\hat{v}_{\infty 2}\right)^{2}-\zeta_{3}^{2}\right) E^{-1}-1, \quad \text { as } r=|x| \rightarrow \infty
$$

with $\hat{v}_{\infty 1}$ and $\hat{v}_{\infty 2}$ given by

$$
\hat{v}_{\infty i}=\frac{v_{\infty i}}{\left(2 R T_{0}\right)^{1 / 2}}, \quad i=1,2
$$

The macroscopic variables are expressed in terms of $\phi$ as follows:

$$
\begin{gathered}
\omega=\langle\phi\rangle, \quad(1+\omega) u_{i}=\left\langle\zeta_{i} \phi\right\rangle, \quad \frac{3}{2}(1+\omega) \tau=\left\langle\left(\zeta^{2}-\frac{3}{2}\right) \phi\right\rangle-(1+\omega) u_{j}^{2}, \\
P=\omega+\tau+\omega \tau, \quad P_{i j}=2\left\langle\zeta_{i} \zeta_{j} \phi\right\rangle-2(1+\omega) u_{i} u_{j}, \\
Q_{i}=\left\langle\zeta_{i} \zeta^{2} \phi\right\rangle-\frac{5}{2} u_{i}-u_{j} P_{i j}-\frac{3}{2} P u_{i}-(1+\omega) u_{i} u_{j}^{2},
\end{gathered}
$$

where the symbol $\langle>$ represents the following integral with respect to $\zeta$ :

$$
\langle g\rangle=\int_{\mathbb{R}^{3}} g(\zeta) E \mathrm{~d} \zeta .
$$

In the actual numerical computations, we employ the BGK model of the Boltzmann equation. The BGK model is obtained by replacing $\mathscr{L}(\phi)$ and $\mathscr{J}(\phi, \phi)$ with the following counterparts (see Appendix A):

$$
\begin{aligned}
\mathscr{L}^{B G K}(\phi) & =g_{e}(\phi)-\phi, \\
\mathscr{J}^{B G K}(\phi) & =(1+\omega)\left(\phi_{e}-\phi\right)-\mathscr{L}^{B G K}(\phi) \\
& =(1+\omega)\left(\phi_{e}-g_{e}\right)+\omega \mathscr{L}^{B G K}(\phi),
\end{aligned}
$$

where

$$
\begin{aligned}
& g_{e}=\langle\phi\rangle+2 \zeta_{i}\left\langle\zeta_{i} \phi\right\rangle+\left(\zeta^{2}-\frac{3}{2}\right) \frac{2}{3}\left\langle\left(\zeta^{2}-\frac{3}{2}\right) \phi\right\rangle, \\
& \phi_{e}=E^{-1} \frac{1+\omega}{\pi^{3 / 2}(1+\tau)^{3 / 2}} \exp \left(-\frac{\left(\zeta_{i}-u_{i}\right)^{2}}{1+\tau}\right)-1,
\end{aligned}
$$

and $\omega, u_{i}$ and $\tau$ are given by $(2.10 a)$. Note that the operators $\mathscr{L}^{B G K}(\phi)$ and $\mathscr{J}^{B G K}(\phi)$ are spherically symmetric. 


\section{S. Taguchi and T. Tsuji}

\subsection{Scaling assumptions}

Now we introduce assumptions (iii) and (iv) in $\S 2.1$ and restrict our consideration to slow flows (i.e. low-Mach-number flows). We thus introduce the quantity

$$
\varepsilon:=\left|\hat{\boldsymbol{v}}_{\infty}\right|=\left(\hat{v}_{\infty 1}^{2}+\hat{v}_{\infty 2}^{2}\right)^{1 / 2},
$$

and assume that $\varepsilon$ is small $(\varepsilon \ll 1)$. Note that the Mach number, mentioned in assumption (iii), is related to $\varepsilon$ by $M a=(6 / 5)^{1 / 2} \varepsilon$. In the next section, we carry out a perturbative analysis for small $\varepsilon$ in the case $k=O(1), \hat{\Omega}_{0}=O(\varepsilon)$ and $|\phi|=O(\varepsilon)$ (i.e. the weakly nonlinear regime). We thus write $\hat{v}_{\infty 1}, \hat{v}_{\infty 2}$ and $\hat{\Omega}_{0}$ as

$$
\hat{v}_{\infty 1}=U \varepsilon=\varepsilon \cos \alpha_{0}, \quad \hat{v}_{\infty 2}=V \varepsilon=\varepsilon \sin \alpha_{0}, \quad \hat{\Omega}_{0}=S \varepsilon,
$$

where $\alpha_{0} \in\left[0,2 \pi\right.$ ) is the azimuth angle of $\boldsymbol{v}_{\infty}$ (see figure 1). Note that $U^{2}+V^{2}=1$ by definition and that (cf. assumption (iv))

$$
S=\frac{\hat{\Omega}_{0}}{\varepsilon}=\frac{L\left|\boldsymbol{\Omega}_{0}\right|}{\left|\boldsymbol{v}_{\infty}\right|}=O(1) .
$$

Then, the boundary conditions are rewritten as

$$
\begin{gathered}
\phi=\frac{1+\sigma_{w}}{\pi^{3 / 2}} \exp \left(-\zeta_{r}^{2}-\zeta_{\theta}^{2}-\left(\zeta_{\varphi}-\varepsilon S \sin \theta\right)^{2}\right) E^{-1}-1, \quad \zeta_{r}>0 \quad(|x|=1), \\
\phi \rightarrow \phi_{\infty}:=\frac{1}{\pi^{3 / 2}} \exp \left(-\left(\zeta_{1}-\varepsilon U\right)^{2}-\left(\zeta_{2}-\varepsilon V\right)^{2}-\zeta_{3}^{2}\right) E^{-1}-1, \quad \text { as }|x| \rightarrow \infty,
\end{gathered}
$$

where $\sigma_{w}$ is given by (2.6).

In summary, the problem to be solved is (2.3), (2.19) with (2.6), and (2.20), where $k, U$, $V$ and $S$ are independent of $\varepsilon$.

Finally, we make the following comment. According to the von Kármán relation (Sone 2007), the Reynolds number $R e=\rho_{0}\left|\boldsymbol{v}_{\infty}\right| L / \mu_{0}$, the Mach number $M a$ and the Knudsen number $K n$ are not independent but are related to each other by the relation $\operatorname{Re} \sim M a / K n$. Here, $\mu_{0}$ is the viscosity of the gas at the reference state. Thus, the present analysis corresponds physically to a situation in which the Reynolds number is small, i.e.

$$
\operatorname{Re} \sim \frac{\varepsilon}{k} \ll 1 .
$$

\section{Asymptotic analysis}

In this section, we carry out an asymptotic analysis of the boundary-value problem (2.3), (2.19) with (2.6), and (2.20) for small $\varepsilon$, with the aim of obtaining expressions for the force and the torque acting on the sphere. The analysis is a straightforward extension of that of Taguchi (2015), in which the case of a non-rotating sphere $(U=1, V=0$ and $S=0)$ is considered. However, the actual calculations are more involved because the flow is no longer axisymmetric.

\subsection{Inner solution}

We first consider a solution to the problem whose length scale of variation is of the order of unity (or of the order of $L$ in dimensional space). We call this length scale the inner 


\section{Inversion of the transverse force on a spinning sphere}

scale for the reason to be clarified below. Accordingly, the solution with this length scale is called the inner solution and hereafter is designated by attaching the subscript $F$, i.e. $\partial \phi_{F} / \partial x_{i}=O\left(\phi_{F}\right)$. We assume that the inner solution can be expanded in $\varepsilon$ as

$$
\phi_{F}=\varepsilon \phi_{F 1}+\varepsilon^{2} \phi_{F 2}+o\left(\varepsilon^{2}\right) .
$$

It may be mentioned that the remainder may not be a simple power series of $\varepsilon$ but contains terms like $\varepsilon^{3} \ln \varepsilon$, as in the Navier-Stokes theory (Proudman \& Pearson 1957; Chester, Breach \& Proudman 1969). In the present study, we will not address this issue further and concentrate on the force exerted on a particle to $\varepsilon^{2}$ order.

Corresponding to the expansion (3.1), the macroscopic variables are also expanded in $\varepsilon$ as

$$
h_{F}=\varepsilon h_{F 1}+\varepsilon^{2} h_{F 2}+o\left(\varepsilon^{2}\right) \quad\left(h=\omega, u_{i}, \tau, P, P_{i j}, Q_{i}\right) .
$$

The relations between $h_{F m}$ and $\phi_{F m}(m=1,2, \ldots)$ are obtained by substituting the expansions of $h_{F}$ and $\phi_{F}$ into the definitions of the macroscopic variables (2.10) with $h=h_{F}$ and $\phi=\phi_{F}$ and by equating terms with the same power of $\varepsilon$. We thus obtain, for the first two orders in $\varepsilon$,

$$
\begin{gathered}
\omega_{F 1}=\left\langle\phi_{F 1}\right\rangle, \quad u_{i F 1}=\left\langle\zeta_{i} \phi_{F 1}\right\rangle, \quad \tau_{F 1}=\frac{2}{3}\left\langle\left(\zeta^{2}-\frac{3}{2}\right) \phi_{F 1}\right\rangle, \\
P_{F 1}=\omega_{F 1}+\tau_{F 1}, \quad P_{i j F 1}=2\left\langle\zeta_{i} \zeta_{j} \phi_{F 1}\right\rangle, \quad Q_{i F 1}=\left\langle\zeta_{i} \zeta^{2} \phi_{F 1}\right\rangle-\frac{5}{2} u_{i F 1}, \\
\omega_{F 2}=\left\langle\phi_{F 2}\right\rangle, \quad u_{i F 2}=\left\langle\zeta_{i} \phi_{F 2}\right\rangle-\omega_{F 1} u_{i F 1}, \\
\tau_{F 2}=\frac{2}{3}\left\langle\left(\zeta^{2}-\frac{3}{2}\right) \phi_{F 2}\right\rangle-\frac{2}{3}\left(u_{j F 1}\right)^{2}-\omega_{F 1} \tau_{F 1}, \\
P_{F 2}=\omega_{F 2}+\tau_{F 2}+\omega_{F 1} \tau_{F 1}, \quad P_{i j F 2}=2\left\langle\zeta_{i} \zeta_{j} \phi_{F 2}\right\rangle-2 u_{i F 1} u_{j F 1}, \\
Q_{i F 2}=\left\langle\zeta_{i} \zeta^{2} \phi_{F 2}\right\rangle-\frac{5}{2} u_{i F 2}-u_{j F 1} P_{i j F 1}-\frac{3}{2} P_{F 1} u_{i F 1} .
\end{gathered}
$$

Note that the nonlinearity enters the relations in the form of a product of lower-order terms in (3.4).

If we substitute the expansion (3.1) into (2.3) and collect terms with the same power of $\varepsilon$, we obtain a sequence of linearized Boltzmann equations for $\phi_{F m}(m=1,2)$, i.e.

$$
\begin{gathered}
\zeta_{i} \frac{\partial \phi_{F 1}}{\partial x_{i}}=\frac{1}{k} \mathscr{L}\left(\phi_{F 1}\right), \\
\zeta_{i} \frac{\partial \phi_{F 2}}{\partial x_{i}}=\frac{1}{k} \mathscr{L}\left(\phi_{F 2}\right)+\frac{1}{k} \mathscr{J}\left(\phi_{F 1}, \phi_{F 1}\right) .
\end{gathered}
$$

Equation (3.5a) is the linearized Boltzmann equation for $\phi_{F 1}$, while (3.5b) is the linearized Boltzmann equation for $\phi_{F 2}$ with an inhomogeneous term. Similarly, if we insert the expansion into the diffuse reflection condition on the sphere, i.e. (2.19) with (2.6), we obtain a sequence of boundary conditions for $\phi_{F m}(m=1,2)$ on the sphere (see (3.7) and (3.65)). Provided appropriate boundary conditions at infinity are given, they form a sequence of boundary-value problems for $\phi_{F m}$, which can be solved successively from the lowest order.

\subsection{Leading order}

The equation and the boundary condition on the sphere for $\phi_{F 1}$ are given by

$$
\zeta_{i} \frac{\partial \phi_{F 1}}{\partial x_{i}}=\frac{1}{k} \mathscr{L}\left(\phi_{F 1}\right),
$$




$$
\begin{gathered}
\text { S. Taguchi and T. Tsuji } \\
\phi_{F 1}=\mathscr{K}\left(\phi_{F 1}\right)+2 S \zeta_{\varphi} \sin \theta, \quad \zeta_{r}>0 \quad(|x|=1),
\end{gathered}
$$

where $\mathscr{K}(\cdot)$ represents

$$
\mathscr{K}(g)=-2 \sqrt{\pi} \int_{\zeta_{r}<0} \zeta_{r} g(\zeta) E \mathrm{~d} \zeta .
$$

To derive the corresponding boundary condition at infinity, we expand $\phi_{\infty}$ in (2.20) as $\phi_{\infty}=\varepsilon \phi_{\infty 1}+\varepsilon^{2} \phi_{\infty 2}+\cdots$ and retain the leading-order term. This yields

$$
\phi_{F 1} \rightarrow 2\left(\zeta_{1} U+\zeta_{2} V\right), \quad \text { as }|x| \rightarrow \infty .
$$

Equations (3.6)-(3.9) form a boundary-value problem of the linearized Boltzmann equation for unknown $\phi_{F 1}$.

In view of the linearity of the problem, we seek the solution in the form

$$
\phi_{F 1}=\Phi_{\mathrm{U}}^{(1)}+\Phi_{\mathrm{S}}^{(1)}
$$

where $\Phi_{\mathrm{U}}^{(1)}$ and $\Phi_{\mathrm{S}}^{(1)}$ solve the following problems:

$$
\begin{gathered}
\zeta_{i} \frac{\partial \Phi_{J}^{(1)}}{\partial x_{i}}=\frac{1}{k} \mathscr{L}\left(\Phi_{J}^{(1)}\right) \quad(J=\mathrm{U}, \mathrm{S}), \\
\Phi_{J}^{(1)}=\mathscr{K}_{\left(\Phi_{J}^{(1)}\right)+I_{w, J}^{(1)}, \quad \zeta_{r}>0, \quad|x|=1,}^{(1)} \rightarrow I_{\infty, J}^{(1)}, \quad \text { as }|x| \rightarrow \infty
\end{gathered}
$$

with

$$
\begin{gathered}
I_{w, \mathrm{U}}^{(1)}=0, \quad I_{w, \mathrm{~S}}^{(1)}=2 \zeta_{\varphi} S \sin \theta \\
I_{\infty, \mathrm{U}}^{(1)}=2 U \zeta_{1}+2 V \zeta_{2}, \quad I_{\infty, \mathrm{S}}^{(1)}=0 .
\end{gathered}
$$

The problem for $\Phi_{\mathrm{S}}^{(1)}$ (hereafter referred to as problem S) describes the steady flow of a rarefied gas around a rotating sphere without any flows at infinity (Loyalka 1992; Andreev \& Popov 2010; Taguchi et al. 2019). The problem for $\Phi_{U}^{(1)}$ (hereafter referred to as problem $\mathrm{U}$ ) is equivalent to the boundary-value problem describing a uniform flow of rarefied gas past a sphere in the absence of sphere rotation, which has been extensively studied in the literature (e.g. Cercignani et al. 1968; Sone \& Aoki 1977a; Takata et al. 1993; Kalempa \& Sharipov 2020; see also Sone 2007).

Using the property of axial symmetry of the operator $\mathscr{L}$ given in Appendix B, we seek $\Phi_{\mathrm{U}}^{(1)}$ and $\Phi_{\mathrm{S}}^{(1)}$ in the forms (i.e. similarity solutions)

$$
\begin{gathered}
\Phi_{\mathrm{U}}^{(1)}=(U \cos \theta+V \sin \theta \cos \varphi) \varphi_{\mathrm{U} a}^{(1)}\left(r, \zeta_{r}, \zeta\right) \\
+\left[\zeta_{\theta}(U \sin \theta-V \cos \theta \cos \varphi)+V \zeta_{\varphi} \sin \varphi\right] \varphi_{\mathrm{U} b}^{(1)}\left(r, \zeta_{r}, \zeta\right), \\
\Phi_{\mathrm{S}}^{(1)}=S \zeta_{\varphi} \sin \theta \varphi_{\mathrm{S}}^{(1)}\left(r, \zeta_{r}, \zeta\right),
\end{gathered}
$$

where the functions $\varphi_{\mathrm{U} a}^{(1)}\left(r, \zeta_{r}, \zeta\right), \varphi_{\mathrm{U} b}^{(1)}\left(r, \zeta_{r}, \zeta\right)$ and $\varphi_{\mathrm{S}}^{(1)}\left(r, \zeta_{r}, \zeta\right)$ solve the following boundary-value problems in space in one dimension (in spherical coordinates): 


$\begin{array}{lccccl}\text { Order } & \text { Problem } & \text { Function } & \begin{array}{c}\text { Functions appearing in } \\ \text { the similarity solution }\end{array} & \text { Definition } & \text { Note } \\ \varepsilon^{1} & \mathrm{U} & \Phi_{\mathrm{U}}^{(1)} & \varphi_{\mathrm{U} a}^{(1)}, \varphi_{\mathrm{U} b}^{(1)} & (3.16 a) & \text { Linear motion } \\ \varepsilon^{1} & \mathrm{~S} & \Phi_{\mathrm{S}}^{(1)} & \varphi_{\mathrm{S}}^{(1)} & (3.16 b) & \text { Rotational motion } \\ \varepsilon^{2} & \mathrm{UU} & \Phi_{\mathrm{UU}}^{(2)} & \varphi_{\mathrm{UU} a}^{(2)}, \varphi_{\mathrm{UU} b}^{(2)}, \varphi_{\mathrm{UU} c}^{(2)}, \varphi_{\mathrm{UU} d}^{(2)} & (3.79) & \text { Linear motion } \\ \varepsilon^{2} & \mathrm{SS} & \Phi_{\mathrm{SS}}^{(2)} & \varphi_{\mathrm{SS} a}^{(2)}, \varphi_{\mathrm{SS} b}^{(2)}, \varphi_{\mathrm{SS} c}^{(2)}, \varphi_{\mathrm{SS} d}^{(2)} & (3.80) & \text { Rotational motion } \\ \varepsilon^{2} & \mathrm{US} & \Phi_{\mathrm{US}}^{(2) \sharp} & \varphi_{\mathrm{US} a}^{(2) \sharp}, \varphi_{\mathrm{US} b}^{(2) \sharp}, \varphi_{\mathrm{US} c}^{(2) \sharp}, \varphi_{\mathrm{US} d}^{(2) \sharp} & (3.82) & \text { Cross effect } \\ \varepsilon^{2} & \mathrm{US} & \Phi_{\mathrm{US}}^{(2) b} & \varphi_{\mathrm{US} a}^{(2) b}, \varphi_{\mathrm{US} b}^{(2) b}, \varphi_{\mathrm{US} c}^{(2) b}, \varphi_{\mathrm{US} d}^{(2) b} & (3.83) & \text { Cross effect }\end{array}$

Table 1. Functions appearing in the similarity solutions.

(a) Problem $U$

$$
\begin{gathered}
\zeta_{r} \frac{\partial \varphi_{\mathrm{U} a}^{(1)}}{\partial r}+\frac{\zeta^{2}-\zeta_{r}^{2}}{r} \frac{\partial \varphi_{\mathrm{U} a}^{(1)}}{\partial \zeta_{r}}+\frac{\zeta^{2}-\zeta_{r}^{2}}{r} \varphi_{\mathrm{U} b}^{(1)}=\frac{1}{k} \mathscr{L}_{0}\left(\varphi_{\mathrm{U} a}^{(1)}\right), \\
\zeta_{r} \frac{\partial \varphi_{\mathrm{U} b}^{(1)}}{\partial r}+\frac{\zeta^{2}-\zeta_{r}^{2}}{r} \frac{\partial \varphi_{\mathrm{U} b}^{(1)}}{\partial \zeta_{r}}-\frac{\zeta_{r}}{r} \varphi_{\mathrm{U} b}^{(1)}-\frac{\varphi_{\mathrm{U} a}^{(1)}}{r}=\frac{1}{k} \mathscr{L}_{1}\left(\varphi_{\mathrm{U} b}^{(1)}\right), \\
\varphi_{\mathrm{U} a}^{(1)}=\mathscr{K}\left(\varphi_{\mathrm{U} a}^{(1)}\right), \quad \varphi_{\mathrm{U} b}^{(1)}=0, \quad \zeta_{r}>0, \quad \text { at } r=1, \\
\varphi_{\mathrm{U} a}^{(1)} \rightarrow 2 \zeta_{r}, \quad \varphi_{\mathrm{U} b}^{(1)} \rightarrow-2, \quad \text { as } r \rightarrow \infty .
\end{gathered}
$$

(b) Problem $S$

$$
\begin{gathered}
\zeta_{r} \frac{\partial \varphi_{\mathrm{S}}^{(1)}}{\partial r}+\frac{\zeta^{2}-\zeta_{r}^{2}}{r} \frac{\partial \varphi_{\mathrm{S}}^{(1)}}{\partial \zeta_{r}}-\frac{\zeta_{r}}{r} \varphi_{\mathrm{S}}^{(1)}=\frac{1}{k} \mathscr{L}_{1}\left(\varphi_{\mathrm{S}}^{(1)}\right), \\
\varphi_{\mathrm{S}}^{(1)}=2, \quad \zeta_{r}>0, \quad \text { at } r=1, \\
\varphi_{\mathrm{S}}^{(1)} \rightarrow 0, \quad \text { as } r \rightarrow \infty .
\end{gathered}
$$

Here, the operators $\mathscr{L}_{0}$ and $\mathscr{L}_{1}$ are defined in Appendix B. Note that $\mathscr{L}_{0}\left(\varphi_{\mathrm{U} a}^{(1)}\right), \mathscr{L}_{1}\left(\varphi_{\mathrm{Ub}}^{(1)}\right)$ and $\mathscr{L}_{1}\left(\varphi_{\mathrm{S}}^{(1)}\right)$ appearing on the right-hand sides of $(3.17 a),(3.17 b)$ and $(3.18 a)$ are functions of $r, \zeta_{r}$ and $\zeta$. For clarity, table 1 summarizes the notation for the similarity solutions.

Suppose that $\varphi_{\mathrm{U} a}^{(1)}$ and $\varphi_{\mathrm{U} b}^{(1)}$ (or $\Phi_{\mathrm{U}}^{(1)}$ ) and $\varphi_{\mathrm{S}}^{(1)}$ (or $\Phi_{\mathrm{S}}^{(1)}$ ) are known. Then, the leading-order macroscopic quantities $\omega_{F 1}, u_{i F 1}$, etc., are obtained by substituting (3.10) with (3.16) into (3.3). To this end, we first introduce the following notation:

$$
\begin{gathered}
\tilde{\omega}[g]=\langle g\rangle, \quad \tilde{u}_{i}[g]=\left\langle\zeta_{i} g\right\rangle, \quad \tilde{\tau}[g]=\frac{2}{3}\left\langle\left(\zeta^{2}-\frac{3}{2}\right) g\right\rangle, \\
\tilde{P}[g]=\frac{2}{3}\left\langle\zeta^{2} g\right\rangle=\tilde{\omega}[g]+\tilde{\tau}[g], \quad \tilde{P}_{i j}[g]=2\left\langle\zeta_{i} \zeta_{j} g\right\rangle, \quad \tilde{Q}_{i}[g]=\left\langle\zeta_{i}\left(\zeta^{2}-\frac{5}{2}\right) g\right\rangle,
\end{gathered}
$$

where $g=g\left(\zeta_{i}\right)$. Note that, for every position $x$ in the gas, $\Phi_{\mathrm{U}}^{(1)}$ and $\Phi_{\mathrm{S}}^{(1)}$ (and thus $\phi_{F 1}$ ) are of the form $g=a_{0} g_{0}\left(\zeta_{r}, \zeta\right)+b_{i} \zeta_{j}\left(\delta_{i j}-\hat{x}_{i} \hat{x}_{j}\right) g_{1}\left(\zeta_{r}, \zeta\right)$, where $\hat{x}_{i}=x_{i} / r=x_{i} /|x|\left(\hat{x}_{i}^{2}=1\right)$ 


\section{S. Taguchi and T. Tsuji}

and $a_{0}$ and $b_{i}$ are independent of $\zeta_{i}$. For such a function, the components of $\tilde{u}_{i}^{g}=\tilde{u}_{i}[g]$, $\tilde{P}_{i j}^{g}=\tilde{P}_{i j}[g]$ and $\tilde{Q}_{i}^{g}=\tilde{Q}_{i}[g]$ in spherical coordinates are calculated as

$$
\left.\begin{array}{c}
\tilde{u}_{r}^{g}=a_{0}\left\langle\zeta_{r} g_{0}\right\rangle, \quad \tilde{u}_{i}^{g} t_{i}=\frac{b_{i} t_{i}}{2}\left\langle\left(\zeta^{2}-\zeta_{r}^{2}\right) g_{1}\right\rangle, \quad \tilde{P}_{r r}^{g}=2 a_{0}\left\langle\zeta_{r}^{2} g_{0}\right\rangle, \\
\tilde{P}_{i j}^{g} t_{i} t_{j}=a_{0}\left\langle\left(\zeta^{2}-\zeta_{r}^{2}\right) g_{0}\right\rangle, \quad \tilde{P}_{r j}^{g} t_{j}=b_{j} t_{j}\left\langle\zeta_{r}\left(\zeta^{2}-\zeta_{r}^{2}\right) g_{1}\right\rangle, \\
\tilde{Q}_{r}^{g}=a_{0}\left\langle\zeta_{r}\left(\zeta^{2}-\frac{5}{2}\right) g_{0}\right\rangle, \quad \tilde{Q}_{i}^{g} t_{i}=\frac{b_{i} t_{i}}{2}\left\langle\left(\zeta^{2}-\zeta_{r}^{2}\right)\left(\zeta^{2}-\frac{5}{2}\right) g_{1}\right\rangle,
\end{array}\right\}
$$

where $t_{i}$ is an arbitrary unit vector perpendicular to $\hat{x}_{i}$. Therefore, if we further introduce the notation

$$
\left.\begin{array}{c}
\tilde{u}_{r}[\varphi]=\left\langle\zeta_{r} \varphi\right\rangle, \quad \tilde{u}_{t}[\varphi]=\frac{1}{2}\left\langle\left(\zeta^{2}-\zeta_{r}^{2}\right) \varphi\right\rangle, \\
\tilde{P}_{r r}[\varphi]=2\left\langle\zeta_{r}^{2} \varphi\right\rangle, \\
\tilde{P}_{t t}[\varphi]=\left\langle\left(\zeta^{2}-\zeta_{r}^{2}\right) \varphi\right\rangle\left(=2 \tilde{u}_{t}[\varphi]\right), \\
\tilde{P}_{r t}[\varphi]=\left\langle\zeta_{r}\left(\zeta^{2}-\zeta_{r}^{2}\right) \varphi\right\rangle,
\end{array}\right\}
$$

where $\varphi=\varphi\left(\zeta_{r}, \zeta\right)$, the leading-order macroscopic quantities (as functions of $(r, \theta, \varphi)$ ) are expressed in the forms

$$
\begin{gathered}
\omega_{F 1}=(U \cos \theta+V \sin \theta \cos \varphi) \tilde{\omega}_{\mathrm{U} a}^{(1)}(r), \\
u_{r F 1}=(U \cos \theta+V \sin \theta \cos \varphi) \tilde{u}_{r, \mathrm{U} a}^{(1)}(r), \\
u_{\theta F 1}=(U \sin \theta-V \cos \theta \cos \varphi) \tilde{u}_{t, \mathrm{U} b}^{(1)}(r), \\
u_{\varphi F 1}=(V \sin \varphi) \tilde{u}_{t, \mathrm{U} b}^{(1)}(r)+(S \sin \theta) \tilde{u}_{t, \mathrm{~S}}^{(1)}(r), \\
\tau_{F 1}=(U \cos \theta+V \sin \theta \cos \varphi) \tilde{\tau}_{\mathrm{U} a}^{(1)}(r), \\
P_{F 1}=(U \cos \theta+V \sin \theta \cos \varphi) \tilde{P}_{\mathrm{U} a}^{(1)}(r), \\
P_{r r F 1}=(U \cos \theta+V \sin \theta \cos \varphi) \tilde{P}_{r r, \mathrm{U} a}^{(1)}(r), \\
P_{r \theta F 1}=(U \sin \theta-V \cos \theta \cos \varphi) \tilde{P}_{r t, \mathrm{U} b}^{(1)}(r), \\
P_{r \varphi F 1}=(V \sin \varphi) \tilde{P}_{r t, \mathrm{U} b}^{(1)}(r)+(S \sin \theta) \tilde{P}_{r t, \mathrm{~S}}^{(1)}(r), \\
P_{\theta \theta F 1}=P_{\varphi \varphi F 1}=(U \cos \theta+V \sin \theta \cos \varphi) \tilde{P}_{t t, \mathrm{U} a}^{(1)}(r), \\
P_{\theta \varphi F 1}=0, \\
Q_{r F 1}=(U \cos \theta+V \sin \theta \cos \varphi) \tilde{Q}_{r, \mathrm{U} a}^{(1)}(r), \\
Q_{\theta F 1}=(U \sin \theta-V \cos \theta \cos \varphi) \tilde{Q}_{t, \mathrm{U} b}^{(1)}(r), \\
Q_{\varphi F 1}=(V \sin \varphi) \tilde{Q}_{t, \mathrm{U} b}^{(1)}(r)+(S \sin \theta) \tilde{Q}_{t, \mathrm{~S}}^{(1)}(r),
\end{gathered}
$$


where

$$
\left.\begin{array}{c}
\tilde{\omega}_{\mathrm{U} a}^{(1)}=\tilde{\omega}\left[\varphi_{\mathrm{U} a}^{(1)}\right], \quad \tilde{u}_{r, \mathrm{U} a}^{(1)}=\tilde{u}_{r}\left[\varphi_{\mathrm{U} a}^{(1)}\right], \quad \tilde{\tau}_{\mathrm{U} a}^{(1)}=\tilde{\tau}\left[\varphi_{\mathrm{U} a}^{(1)}\right], \quad \tilde{P}_{\mathrm{U} a}^{(1)}=\tilde{P}\left[\varphi_{\mathrm{U} a}^{(1)}\right], \\
\tilde{P}_{r r, \mathrm{U} a}^{(1)}=\tilde{P}_{r r}\left[\varphi_{\mathrm{U} a}^{(1)}\right], \quad \tilde{P}_{t t, \mathrm{U} a}^{(1)}=\tilde{P}_{t t}\left[\varphi_{\mathrm{U} a}^{(1)}\right], \quad \tilde{Q}_{r, \mathrm{U} a}^{(1)}=\tilde{Q}_{r}\left[\varphi_{\mathrm{U} a}^{(1)}\right], \\
\tilde{u}_{t, \mathrm{U} b}^{(1)}=\tilde{u}_{t}\left[\varphi_{\mathrm{U} b}^{(1)}\right], \quad \tilde{P}_{r t, \mathrm{U} b}^{(1)}=\tilde{P}_{r t}\left[\varphi_{\mathrm{U} b}^{(1)}\right], \quad \tilde{Q}_{t, \mathrm{U} b}^{(1)}=\tilde{Q}_{t}\left[\varphi_{\mathrm{U} b}^{(1)}\right], \\
\tilde{u}_{t, \mathrm{~S}}^{(1)}=\tilde{u}_{t}\left[\varphi_{\mathrm{S}}^{(1)}\right], \quad \tilde{P}_{r t, \mathrm{~S}}^{(1)}=\tilde{P}_{r t}\left[\varphi_{\mathrm{S}}^{(1)}\right], \quad \tilde{Q}_{t, \mathrm{~S}}^{(1)}=\tilde{Q}_{t}\left[\varphi_{\mathrm{S}}^{(1)}\right] .
\end{array}\right\}
$$

Note that $\tilde{\omega}_{\mathrm{U} a}^{(1)}, \tilde{u}_{r, \mathrm{U} a}^{(1)}$, etc., depend on $r$ through $\varphi_{\mathrm{U} a}^{(1)}, \varphi_{\mathrm{U} b}^{(1)}$ and $\varphi_{\mathrm{S}}^{(1)}$, as shown explicitly in (3.22). We will not repeat similar comments in what follows.

Finally, let us consider the force and the torque acting on the sphere. Let $p_{0} L^{2} \mathcal{F}_{i}$ and $p_{0} L^{3} \mathcal{M}_{i}$ denote, respectively, the force and moment of force (about the origin) acting on the sphere. Then, $\mathcal{F}_{i}$ and $\mathcal{M}_{i}$ are given in terms of the stress tensor as

$$
\mathcal{F}_{i}=-\int_{|x|=1} P_{i j F} n_{j} \mathrm{~d} S, \quad \mathcal{M}_{i}=-\int_{|x|=1} \epsilon_{i j k} x_{j} P_{k l F} n_{l} \mathrm{~d} S,
$$

where $\mathrm{d} S$ is the surface element, $n_{i}$ is the unit normal vector on the sphere pointing to the gas, $\epsilon_{i j k}(i, j, k=1,2,3)$ is the Eddington epsilon (the permutation symbol) and the integration is carried out over the whole surface $|x|=1$. We expand the (dimensionless) force and torque in $\varepsilon$ as

$$
\mathcal{F}_{i}=\varepsilon \mathcal{F}_{i}^{(1)}+\varepsilon^{2} \mathcal{F}_{i}^{(2)}+o\left(\varepsilon^{2}\right), \quad \mathcal{M}_{i}=\varepsilon \mathcal{M}_{i}^{(1)}+\varepsilon^{2} \mathcal{M}_{i}^{(2)}+o\left(\varepsilon^{2}\right),
$$

where $\mathcal{F}_{i}^{(m)}$ and $\mathcal{M}_{i}^{(m)}, m=1,2$, are given by

$$
\mathcal{F}_{i}^{(m)}=-\int_{|x|=1} P_{i j F m} n_{j} \mathrm{~d} S, \quad \mathcal{M}_{i}^{(m)}=-\int_{|x|=1} \epsilon_{i j k} x_{j} P_{k l F m} n_{l} \mathrm{~d} S .
$$

Substituting (3.22g)-(3.22i) into $(3.26 a, b)$ with $m=1$, the force $\mathcal{F}_{i}^{(1)}$ and the moment of force $\mathcal{M}_{i}^{(1)}$ are obtained as

$$
\begin{gathered}
\mathcal{F}_{1}^{(1)}=U h_{D}, \quad \mathcal{F}_{2}^{(1)}=V h_{D}, \quad \mathcal{F}_{3}^{(1)}=0, \\
\mathcal{M}_{1}^{(1)}=S h_{M}, \quad \mathcal{M}_{2}^{(1)}=0, \quad \mathcal{M}_{3}^{(1)}=0,
\end{gathered}
$$

where

$$
\begin{gathered}
h_{D}=-\left.\frac{4}{3} \pi\left(\tilde{P}_{r r, \mathrm{U} a}^{(1)}-2 \tilde{P}_{r t, \mathrm{U} b}^{(1)}\right)\right|_{r=1}, \\
h_{M}=-\left.\frac{8}{3} \pi \tilde{P}_{r t, \mathrm{~S}}^{(1)}\right|_{r=1} .
\end{gathered}
$$

Introducing the two unit vectors

$$
\left(e_{i}\right)_{i=1,2,3}=(U, V, 0), \quad\left(\hat{e}_{i}\right)_{i=1,2,3}=(1,0,0),
$$

the force and the torque acting on the sphere are summarized as

$$
\mathcal{F}_{i}=\varepsilon h_{D} e_{i}+O\left(\varepsilon^{2}\right), \quad \mathcal{M}_{i}=\varepsilon S h_{M} \hat{e}_{i}+O\left(\varepsilon^{2}\right) .
$$

Thus, no transverse force acts on the sphere at leading order, i.e. $\mathcal{F}_{3}=O\left(\varepsilon^{2}\right)$. It should be noted that $h_{D}$ and $h_{M}$ depend on $k$ through $\varphi_{\mathrm{U} a}^{(1)}, \varphi_{\mathrm{U} b}^{(1)}$ and $\varphi_{\mathrm{S}}^{(1)}$. Therefore, we write 


\section{S. Taguchi and T. Tsuji}

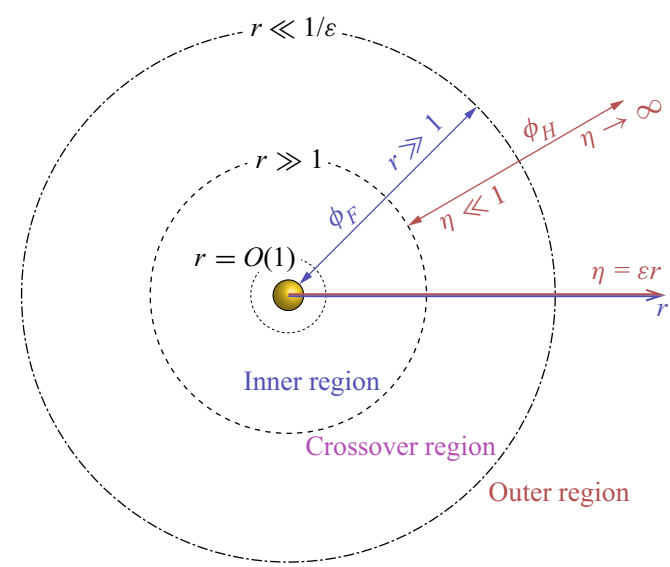

Figure 2. Schematic of the solution structure. The gas region is divided into two, the inner region $1<r \ll \varepsilon^{-1}$ and the outer region $1 \ll r<\infty$, overlapping each other in the crossover region $1 \ll r \ll \varepsilon^{-1}$.

$h_{D}=h_{D}(k)$ and $h_{M}=h_{M}(k)$. In other words, the magnitudes of the force and torque vary with $k$.

To summarize, the sphere is subject to a drag force but no transverse force acts on the sphere at the order $\varepsilon$. The drag and torque are modulated by the Knudsen number through the functions $h_{D}(k)$ and $h_{M}(k)$ given by (3.28) and (3.29), respectively.

\subsection{Slowly varying solution}

In the preceding section, we considered the leading-order problem under the condition that the length scale of variation of the solution is of the order of unity. The solution does not support a transverse force on the sphere. Thus, we are motivated to proceed to the next-order problem in $\varepsilon$.

We note that the linearized Boltzmann equation considered in the preceding subsection may not provide an approximate solution to the original (nonlinear) problem for small $\varepsilon$ uniformly in space. Indeed, using the asymptotic representation of $\phi_{F 1}=\Phi_{\mathrm{U}}^{(1)}+\Phi_{\mathrm{S}}^{(1)}$ for $r=|x| \gg 1$ (see Proposition 3.1 given below), it can be shown that the linearization is valid in the region $|x| \ll \varepsilon^{-1}$ for $\Phi_{U}$, although no such restriction is found for $\Phi_{S}$. In the region beyond this range, the nonlinear term $\mathscr{J}\left(\varepsilon \Phi_{\mathrm{U}}, \varepsilon \Phi_{\mathrm{U}}\right)$, integrated over a long distance, gives a non-negligible contribution to the behaviour of $\varepsilon \Phi_{\mathrm{U}}$. (Note that the term $\zeta_{i} \partial\left(\varepsilon \Phi_{\mathrm{U}}\right) / \partial x_{i}$ is comparable with the nonlinear term $\mathscr{J}\left(\varepsilon \Phi_{\mathrm{U}}, \varepsilon \Phi_{\mathrm{U}}\right)$ when the length scale of variation of $\Phi_{U}$ is of the order of $\varepsilon^{-1}$.) In other words, we encounter a situation analogous to Whitehead's paradox in the Navier-Stokes theory (Van Dyke 1975; Taguchi 2015).

Given this observation, we introduce another length scale to describe the solution in the far region. More specifically, from now on, we assume a solution whose length scale of variation is of the order of $1 / \varepsilon$ in the far region. We call this solution the slowly varying solution (or the outer solution) and designate it by attaching the subscript $H$, i.e. $\partial \phi_{H} / \partial x_{i}=O\left(\varepsilon \phi_{H}\right)$. The situation is schematically shown in figure 2 .

To analyse the slowly varying solution, it is convenient to introduce a new spatial variable (called the outer or slow variable) by

$$
y_{i}=\varepsilon x_{i}
$$


and assume that $\phi_{H}=\phi_{H}\left(y_{i}, \zeta_{i}\right)$. Then, the Boltzmann equation for $\phi_{H}$ is recast as

$$
\zeta_{i} \frac{\partial \phi_{H}}{\partial y_{i}}=\frac{1}{k \varepsilon} \mathscr{L}\left(\phi_{H}\right)+\frac{1}{k \varepsilon} \mathscr{J}\left(\phi_{H}, \phi_{H}\right) .
$$

We seek a solution to (3.33) in the form of a power series in $\varepsilon$, i.e.

$$
\phi_{H}=\varepsilon \phi_{H 1}+\varepsilon^{2} \phi_{H 2}+\cdots .
$$

Likewise, the macroscopic quantities $h_{H}\left(h=\omega, u_{i}, \tau\right.$, etc. $)$ are expanded in $\varepsilon$ as

$$
h_{H}=\varepsilon h_{H 1}+\varepsilon^{2} h_{H 2}+\cdots \text {. }
$$

The relations between $h_{H m}$ and $\phi_{H m}$ are the same as those between $h_{F m}$ and $\phi_{F m}$ except that the subscript should be changed from $F$ to $H$ (see (3.3) and (3.4)).

The above expansion for $\phi_{H}$ is a Hilbert-type expansion starting from the order $\varepsilon$, which is equivalent to the $\mathrm{S}$ expansion (Sone 1971, 2002, 2007). Since a detailed description of the expansion is given in Sone $(2002,2007)$, we only give the results necessary for the subsequent analysis, omitting the derivation.

\subsubsection{Fluid-dynamic-type equations}

First, we summarize fluid-dynamic-type equations describing the behaviour of the gas in the far region. That is, the macroscopic quantities $h_{H m}, m=1,2, \ldots$, are described by the following (incompressible) Navier-Stokes-type equations (hereafter, we call them the Navier-Stokes equations).

Order $\varepsilon$ :

$$
\begin{gathered}
\frac{\partial P_{H 1}}{\partial y_{i}}=0 \\
\frac{\partial u_{j H 1}}{\partial y_{j}}=0 \\
u_{j H 1} \frac{\partial u_{i H 1}}{\partial y_{j}}=-\frac{1}{2} \frac{\partial P_{H 2}}{\partial y_{i}}+\frac{\gamma_{1} k}{2} \Delta u_{i H 1}, \\
u_{j H 1} \frac{\partial \tau_{H 1}}{\partial y_{j}}=\frac{\gamma_{2} k}{2} \Delta \tau_{H 1}, \\
\omega_{H 1}=P_{H 1}-\tau_{H 1} .
\end{gathered}
$$

Order $\varepsilon^{2}$ :

$$
\begin{gathered}
\frac{\partial u_{j H 2}}{\partial y_{j}}=-u_{j H 1} \frac{\partial \omega_{H 1}}{\partial y_{j}}, \\
u_{j H 1} \frac{\partial u_{i H 2}}{\partial y_{j}}+\left(\omega_{H 1} u_{j H 1}+u_{j H 2}\right) \frac{\partial u_{i H 1}}{\partial y_{j}} \\
=-\frac{1}{2} \frac{\partial}{\partial y_{i}}\left(P_{H 3}-\frac{\gamma_{1} \gamma_{2}-4 \gamma_{3}}{6} k^{2} \Delta \tau_{H 1}\right) \\
+\frac{\gamma_{1} k}{2} \Delta u_{i H 2}+\frac{\gamma_{4} k}{2} \frac{\partial}{\partial y_{j}}\left[\tau_{H 1}\left(\frac{\partial u_{i H 1}}{\partial y_{j}}+\frac{\partial u_{j H 1}}{\partial y_{i}}\right)\right],
\end{gathered}
$$




\section{S. Taguchi and T. Tsuji}

HS

BGK

Note

$\begin{array}{lcl}\gamma_{1} & 1.270042427 & 1 \\ \gamma_{2} & 1.922284066 & 1 \\ \gamma_{3} & 1.947906335 & 1 \\ \gamma_{4} & 0.635021 & 1 \\ \gamma_{5} & 0.961142 & 1\end{array}$

Viscosity

Thermal conductivity

Thermal stress

Table 2. The numerical values of $\gamma_{i}$ for a hard-sphere gas (Sone 2002, 2007). The values for the BGK model are also shown.

$$
\begin{gathered}
u_{j H 1} \frac{\partial \tau_{H 2}}{\partial y_{j}}+\left(\omega_{H 1} u_{j H 1}+u_{j H 2}\right) \frac{\partial \tau_{H 1}}{\partial y_{j}}-\frac{2}{5} u_{j H 1} \frac{\partial P_{H 2}}{\partial y_{j}} \\
=\frac{\gamma_{1} k}{5}\left(\frac{\partial u_{i H 1}}{\partial y_{j}}+\frac{\partial u_{j H 1}}{\partial y_{i}}\right)^{2}+\frac{k}{2} \Delta\left(\gamma_{2} \tau_{H 2}+\frac{\gamma_{5}}{2}\left(\tau_{H 1}\right)^{2}\right), \\
\omega_{H 2}=P_{H 2}-\tau_{H 2}-\omega_{H 1} \tau_{H 1} .
\end{gathered}
$$

Here, $\Delta=\partial^{2} / \partial y_{j}^{2}$ is the Laplacian operator and $\gamma_{i}, i=1, \ldots, 5$, are constants defined by

$$
\begin{gathered}
\gamma_{1}=\frac{2}{15}\left\langle\zeta^{4} B\right\rangle, \quad \gamma_{2}=\frac{4}{15}\left\langle\zeta^{4} A\right\rangle \\
\gamma_{3}=\frac{2}{15}\left\langle\zeta^{4} A B\right\rangle=\frac{2}{3}\left\langle\zeta^{4} D_{1}\right\rangle+\frac{2}{15}\left\langle\zeta^{6} D_{2}\right\rangle=-\frac{4}{15}\left\langle\zeta^{4} F\right\rangle, \\
\gamma_{4}=-\frac{5}{2} \gamma_{1}+\frac{2}{15}\left\langle\zeta^{6} B\right\rangle+\frac{1}{15}\left\langle\zeta^{4} B C\right\rangle \\
\gamma_{5}=-6 \gamma_{2}+\frac{4}{15}\left\langle\zeta^{6} A\right\rangle+\frac{4}{15}\left\langle\zeta^{2} A G\right\rangle
\end{gathered}
$$

where $A(\zeta), B(\zeta), C(\zeta), D_{1}(\zeta), D_{2}(\zeta), F(\zeta)$ and $G(\zeta)$ are functions defined in Appendix C. Physically, $\gamma_{i}$ are dimensionless transport coefficients. For example, the viscosity $\mu_{0}$ and the thermal conductivity $\lambda_{0}$ at the reference equilibrium state at rest are expressed as

$$
\mu_{0}=\frac{\sqrt{\pi}}{2} \gamma_{1} \frac{p_{0} \ell_{0}}{\left(2 R T_{0}\right)^{1 / 2}}, \quad \lambda_{0}=\frac{5}{4} \sqrt{\pi} \gamma_{2} \frac{R p_{0} \ell_{0}}{\left(2 R T_{0}\right)^{1 / 2}} .
$$

The numerical value of $\gamma_{i}$ depends on the molecular model. For the BGK model, $\gamma_{i}=1$. The values of $\gamma_{i}$ for the hard-sphere model are summarized in table 2 .

\subsubsection{Velocity distribution functions and boundary conditions for the Navier-Stokes system at infinity}

Suppose that the macroscopic variables $P_{H m}, u_{i H m}$ and $\tau_{H m}(m \geq 1)$ satisfy the fluid-dynamic-type equations (3.36)-(3.38). Then, $\phi_{H m}, m=1,2$, are expressed using $P_{H n}, u_{i H n}$ and $\tau_{H n}(n=1,2)$ as

$$
\begin{gathered}
\phi_{H 1}=\phi_{e H 1} \\
\phi_{H 2}=\phi_{e H 2}-\frac{1}{2} k \zeta_{i} \zeta_{j} B(\zeta)\left(\frac{\partial u_{i H 1}}{\partial y_{j}}+\frac{\partial u_{j H 1}}{\partial y_{i}}\right)-k \zeta_{i} A(\zeta) \frac{\partial \tau_{H 1}}{\partial y_{i}}
\end{gathered}
$$


where

$$
\begin{gathered}
\phi_{e H 1}=P_{H 1}+2 \zeta_{i} u_{i H 1}+\left(\zeta^{2}-\frac{5}{2}\right) \tau_{H 1} \\
\phi_{e H 2}=P_{H 2}+2 \zeta_{i} u_{i H 2}+\left(\zeta^{2}-\frac{5}{2}\right) \tau_{H 2}+2 \zeta_{i} u_{i H 1} P_{H 1}+\left(\zeta^{2}-\frac{5}{2}\right) \tau_{H 1} P_{H 1} \\
+\frac{2}{3}\left(\zeta^{2}-\frac{3}{2}\right)\left(u_{j H 1}\right)^{2}+2\left(\zeta_{i} \zeta_{j}-\frac{\zeta^{2}}{3} \delta_{i j}\right) u_{i H 1} u_{j H 1}+2 \zeta_{i}\left(\zeta^{2}-\frac{7}{2}\right) u_{i H 1} \tau_{H 1} \\
+\frac{1}{2}\left(\zeta^{4}-7 \zeta^{2}+\frac{35}{4}\right)\left(\tau_{H 1}\right)^{2}
\end{gathered}
$$

and the functions $A(\zeta)$ and $B(\zeta)$ are defined in Appendix C. It should be noted that $\phi_{e H 1}$ and $\phi_{e H 2}$ are the first two terms of the expansion $\phi_{e H}=\varepsilon \phi_{e H 1}+\varepsilon^{2} \phi_{e H 2}+\cdots$ of the Maxwellian

$$
\left(1+\phi_{e H}\right) E=\frac{1+P_{H}}{\pi^{3 / 2}\left(1+\tau_{H}\right)^{5 / 2}} \exp \left(-\frac{\left(\zeta_{i}-u_{i H}\right)^{2}}{1+\tau_{H}}\right),
$$

obtained by inserting the expansions of $P_{H}, u_{i H}$ and $\tau_{H}$ in $\varepsilon$ (see (3.35)). Therefore, for $\phi_{H}$ to satisfy the boundary condition at infinity, i.e. (2.20) with $\phi=\phi_{H}$, the macroscopic variables contained in $\phi_{H 1}$ and $\phi_{H 2}$ should satisfy the following conditions:

$$
\begin{gathered}
P_{H 1} \rightarrow 0, \quad u_{i H 1} \rightarrow e_{i}=(U, V, 0), \quad \tau_{H 1} \rightarrow 0, \quad \text { as } \eta \rightarrow \infty, \\
P_{H 2} \rightarrow 0, \quad u_{i H 2} \rightarrow 0, \quad \tau_{H 2} \rightarrow 0, \quad \text { as } \eta \rightarrow \infty
\end{gathered}
$$

where

$$
\eta=\varepsilon r=|\boldsymbol{y}| \quad(\text { outer variable }) .
$$

These conditions serve as a part of the boundary conditions for the Navier-Stokes equations. The remaining conditions are derived by matching the outer solution with the inner solution, as shown next.

\subsection{Outer problem}

In the preceding subsection, we introduced a slowly varying solution characterized by the longer length scale of variation. This solution is meaningful only if it can be matched with the inner solution while meeting the boundary condition at infinity. This subsection shows that it is indeed the case and we determine the first two terms of the $\varepsilon$ expansion of $\phi_{H}$.

\subsubsection{Preliminary}

We begin with the following results.

Proposition 3.1. Let $\left(\varphi_{\mathrm{U} a}^{(1)}, \varphi_{\mathrm{U} b}^{(1)}\right)$ be a solution to the boundary-value problem (3.17) for $k<\infty$ and let $\varphi_{\mathrm{S}}^{(1)}$ be a solution to the boundary-value problem (3.18) for $k<\infty$. 


\section{S. Taguchi and T. Tsuji}

Then, they have the following asymptotic representations as $r \rightarrow \infty$ :

$$
\begin{aligned}
\varphi_{\mathrm{U} a}^{(1)}= & 2 \zeta_{r}\left(1+\frac{c_{1}}{r}+\frac{c_{2}}{r^{3}}\right)+\left(\zeta^{2}-\frac{5}{2}\right) \frac{c_{3}}{r^{2}} \\
& +\frac{k}{r^{2}}\left[\gamma_{1} c_{1}+\frac{2 c_{3}}{r} \zeta_{r} A(\zeta)-\frac{1}{2}\left(c_{1}+\frac{3 c_{2}}{r^{2}}\right)\left(\zeta^{2}-3 \zeta_{r}^{2}\right) B(\zeta)\right] \\
& -\frac{k^{2}}{r^{3}}\left\{2 c_{1} \zeta_{r} D_{1}(\zeta)+2\left[c_{1}\left(2 \zeta^{2}-3 \zeta_{r}^{2}\right)+\frac{3 c_{2}}{r^{2}}\left(3 \zeta^{2}-5 \zeta_{r}^{2}\right)\right] \zeta_{r} D_{2}(\zeta)\right. \\
& \left.-\frac{3 c_{3}}{r}\left(\zeta^{2}-3 \zeta_{r}^{2}\right) F(\zeta)\right\}, \\
\varphi_{\mathrm{U} b}^{(1)}= & -2-\frac{c_{1}}{r}+\frac{c_{2}}{r^{3}}+\frac{k}{r^{3}}\left(c_{3} A(\zeta)+\frac{3 c_{2}}{r} \zeta_{r} B(\zeta)\right) \\
& -\frac{k^{2}}{r^{3}}\left\{c_{1} D_{1}(\zeta)+\left[\frac{c_{1}}{2}\left(\zeta^{2}-3 \zeta_{r}^{2}\right)+\frac{9 c_{2}}{2 r^{2}}\left(\zeta^{2}-5 \zeta_{r}^{2}\right)\right] D_{2}(\zeta)\right. \\
& \left.+\frac{6 c_{3}}{r} \zeta_{r} F(\zeta)\right\}, \\
\varphi_{\mathrm{S}}^{(1)}= & \frac{c_{4}}{r^{2}}\left[2+\frac{3 k}{r} \zeta_{r} B(\zeta)-\frac{3 k^{2}}{r^{2}}\left(\zeta^{2}-5 \zeta_{r}^{2}\right) D_{2}(\zeta)\right]
\end{aligned}
$$

where $c_{i}, i=1,2,3,4$, are constants independent of $r, \zeta_{r}$ and $\zeta$, and $A(\zeta), B(\zeta), D_{1}(\zeta)$, $D_{2}(\zeta)$ and $F(\zeta)$ are the solutions to the integral equations $(\mathrm{C} 1)$ with the subsidiary conditions (C2), given in Appendix C. The constant $\gamma_{1}$ is defined in (3.39a).

It should be noted that $c_{i}$ depends on $k$. The next proposition relates $c_{1}$ and $c_{4}$ with $h_{D}$ and $h_{M}$.

Proposition 3.2. Constants $c_{1}$ and $c_{4}$ are related to $h_{D}$ and $h_{M}$, defined in (3.28) and (3.29), by

$$
c_{1}=-\frac{h_{D}}{4 \pi \gamma_{1} k}, \quad c_{4}=-\frac{h_{M}}{8 \pi \gamma_{1} k} \quad(0<k<\infty) .
$$

The proofs of Propositions 3.1 and 3.2 are given in Appendix D. We also mention that the constant $c_{3}$ is related to the thermophoretic force acting on a single sphere (Taguchi \& Suzuki 2017).

\subsubsection{Leading- and second-order outer solutions}

Let us consider an arbitrary point $\boldsymbol{x}$ in the domain such that $r=|\boldsymbol{x}| \gg 1$ and $r=|\boldsymbol{x}| \ll$ $1 / \varepsilon$. From Proposition 3.1 , the leading-order inner solution at this point is approximated by

$$
\begin{gathered}
\varphi_{\mathrm{U} a}^{(1)}\left(r, \zeta_{r}, \zeta\right)=2 \zeta_{r}\left(1+\frac{c_{1}}{r}\right)+O\left(r^{-2}\right), \quad \varphi_{\mathrm{U} b}^{(1)}\left(r, \zeta_{r}, \zeta\right)=-2-\frac{c_{1}}{r}+O\left(r^{-3}\right), \\
\varphi_{\mathrm{S}}^{(1)}\left(r, \zeta_{r}, \zeta\right)=O\left(r^{-2}\right)
\end{gathered}
$$




\section{Inversion of the transverse force on a spinning sphere}

or equivalently by

$$
\begin{aligned}
\phi_{F 1}= & 2 \zeta_{1} U+2 \zeta_{2} V \\
& +2 \zeta_{r}(U \cos \theta+V \sin \theta \cos \varphi) \frac{c_{1}}{r}-\zeta_{\theta}(U \sin \theta-V \cos \theta \cos \varphi) \frac{c_{1}}{r} \\
& -\zeta_{\varphi} V \sin \varphi \frac{c_{1}}{r}+O\left(r^{-2}\right),
\end{aligned}
$$

using (3.10) and (3.16). Thus, the inner solution has a far-field asymptotic representation of the form

$$
\phi_{F}=\varepsilon\left(2 \zeta_{1} U+2 \zeta_{2} V+\frac{1}{r}(\cdots)+O\left(r^{-2}\right)\right)+\cdots, \quad r=|x| \gg 1,
$$

where the part ' $(\cdots)$ ' in the parentheses is independent of $r$ and $\varepsilon$. Now we consider the limit $\varepsilon \searrow 0$ (and $r \nearrow \infty$ ) with $\varepsilon r(=\eta)$ fixed. Substituting $r=\eta / \varepsilon$ into the above expression and arranging the resulting terms in increasing order of $\varepsilon$, we obtain an $\varepsilon$ expansion of $\phi_{F}$ of the form

$$
\begin{aligned}
\phi_{F}^{*}= & \varepsilon\left(2 \zeta_{1} U+2 \zeta_{2} V\right) \\
& +\varepsilon^{2}\left[2 \zeta_{r}(U \cos \theta+V \sin \theta \cos \varphi)-\zeta_{\theta}(U \sin \theta-V \cos \theta \cos \varphi)-\zeta_{\varphi} V \sin \varphi\right] \frac{c_{1}}{\eta} \\
& +\cdots, \quad \text { as } \varepsilon \searrow 0 \text { with } \varepsilon r(=\eta) \text { fixed. }
\end{aligned}
$$

Here, we have attached ${ }^{*}$ to indicate that $\phi_{F}$ is expressed using the outer variable $\eta$ (or $y_{i}$ ). Thus, a term-by-term comparison between (3.41) (with (3.42)) and (3.54) shows that $\phi_{H}$ can be made to match $\phi_{F}^{*}$ by imposing the following conditions:

$$
\begin{gathered}
P_{H 1} \rightarrow 0, \quad u_{i H 1} \rightarrow(U, V, 0), \quad \tau_{H 1} \rightarrow 0, \\
P_{H 2} \rightarrow 0, \\
u_{r H 2} \rightarrow(U \cos \theta+V \sin \theta \cos \varphi) \frac{c_{1}}{\eta}, \\
u_{\theta H 2} \rightarrow-(U \sin \theta-V \cos \theta \cos \varphi) \frac{c_{1}}{2 \eta}, \\
u_{\varphi H 2} \rightarrow-V \sin \varphi \frac{c_{1}}{2 \eta}, \\
\tau_{H 2} \rightarrow 0,
\end{gathered}
$$

as $\eta \rightarrow 0_{+}$. Together with the conditions (3.44) and (3.45), these conditions serve as appropriate boundary conditions for the Navier-Stokes equations (3.36)-(3.38).

The leading-order problem, (3.36), (3.37), (3.44) and (3.55), is trivially solved by

$$
u_{i H 1}=e_{i}, \quad P_{H 1}=\tau_{H 1}=\omega_{H 1}=0, \quad P_{H 2}=0 .
$$

(Note that $P_{H 2}$ is determined up to an additive constant but this constant can be made to vanish by using the condition (3.56a).) Then, the next-order set of equations (3.38) reduce to the following system of linear partial differential equations:

$$
\frac{\partial u_{j H 2}}{\partial y_{j}}=0,
$$




\section{S. Taguchi and T. Tsuji}

$$
\begin{gathered}
e_{j} \frac{\partial u_{i H 2}}{\partial y_{j}}=-\frac{1}{2} \frac{\partial P_{H 3}}{\partial y_{i}}+\frac{\gamma_{1} k}{2} \Delta u_{i H 2}, \\
e_{j} \frac{\partial \tau_{H 2}}{\partial y_{j}}=\frac{\gamma_{2} k}{2} \Delta \tau_{H 2}, \\
\omega_{H 2}=-\tau_{H 2},
\end{gathered}
$$

which, equipped with the boundary conditions (3.45) and (3.56b)-(3.56e), describes the behaviour of $u_{i H 2}, P_{H 3}, \tau_{H 2}$ and $\omega_{H 2}$ in the far region. We first note that $\tau_{H 2}=0$ is the trivial solution of (3.58c) satisfying (3.56e) and the last condition of (3.45). Next, (3.58b) with (3.58a) is the Oseen equation for incompressible flow, and the solution subject to the boundary conditions (3.56b)-(3.56d) and (3.45) is easily obtained (e.g. Rubinow \& Keller 1961).

Here, we summarize the second-order macroscopic quantities in the outer region. They are given by

$$
\begin{gathered}
u_{r H 2}=\frac{c_{1} \gamma_{1} k}{2} \frac{1}{\eta^{2}}\left[-1+\left(1+\frac{\eta}{\gamma_{1} k}(1+U \cos \theta+V \sin \theta \cos \varphi)\right)\right. \\
\left.\quad \times \exp \left(-\frac{\eta}{\gamma_{1} k}(1-U \cos \theta-V \sin \theta \cos \varphi)\right)\right] \\
u_{\theta H 2}=-\frac{c_{1}}{2 \eta}(U \sin \theta-V \cos \theta \cos \varphi) \exp \left(-\frac{\eta}{\gamma_{1} k}(1-U \cos \theta-V \sin \theta \cos \varphi)\right), \\
u_{\varphi H 2}=-\frac{c_{1}}{2 \eta} V \sin \varphi \exp \left(-\frac{\eta}{\gamma_{1} k}(1-U \cos \theta-V \sin \theta \cos \varphi)\right), \\
P_{H 3}=\frac{\omega_{H 2}=\tau_{H 2}=P_{H 2}=0}{\eta^{2}}(U \cos \theta+V \sin \theta \cos \varphi) .
\end{gathered}
$$

Note that $\left(u_{r H 2}, u_{\theta H 2}, u_{\varphi H 2}\right)$ are proportional to the $k$-dependent constant $c_{1}$.

Finally, we can readily obtain the explicit forms of $\phi_{H 1}$ and $\phi_{H 2}$ from (3.41a) and (3.41b) by substituting $(3.57 a-c)$ and (3.59). In particular, the outer solution is a Maxwellian to order $\varepsilon^{2}$, i.e.

$$
\left(1+\phi_{H}\right) E=\frac{1}{\pi^{3 / 2}} \exp \left(-\left(\zeta_{i}-\varepsilon u_{i H 1}-\varepsilon^{2} u_{i H 2}\right)^{2}\right)\left(1+o\left(\varepsilon^{2}\right)\right)
$$

\subsection{Second-order inner problem}

Now we return to the near region and consider the second-order approximation, i.e. $\phi_{F 2}$. As before, we consider an arbitrary point $x$ such that $|x| \gg 1$ and $|x| \ll 1 / \varepsilon$. Because $\left(u_{r H 2}, u_{\theta H 2}, u_{\varphi H 2}\right)$ are expanded for small $\eta \ll 1$ as

$$
\begin{aligned}
u_{r H 2}(\eta, \theta, \varphi)= & \frac{c_{1}}{\eta}(U \cos \theta+V \sin \theta \cos \varphi) \\
& +\frac{c_{1}}{4 \gamma_{1} k}(U \cos \theta+V \sin \theta \cos \varphi-1)[3(U \cos \theta+V \sin \theta \cos \varphi)+1] \\
& +O(\eta),
\end{aligned}
$$


Inversion of the transverse force on a spinning sphere

$$
\begin{aligned}
u_{\theta H 2}(\eta, \theta, \varphi)= & -\frac{c_{1}}{2 \eta}(U \sin \theta-V \cos \theta \cos \varphi) \\
& -\frac{c_{1}}{2 \gamma_{1} k}(U \sin \theta-V \cos \theta \cos \varphi)(U \cos \theta+V \sin \theta \cos \varphi-1) \\
& +O(\eta), \\
u_{\varphi H 2}(\eta, \theta, \varphi)= & -\frac{c_{1}}{2 \eta} V \sin \varphi-\frac{c_{1}}{2 \gamma_{1} k} V \sin \varphi(U \cos \theta+V \sin \theta \cos \varphi-1) \\
& +O(\eta),
\end{aligned}
$$

the outer solution $\phi_{H}=\varepsilon \phi_{H 1}+\varepsilon^{2} \phi_{H 2}+\cdots$ has the following expansion in terms of the inner variable:

$$
\begin{aligned}
\phi_{H}^{*}= & \varepsilon\left[2\left(\zeta_{1} U+\zeta_{2} V\right)+2 \zeta_{r}(U \cos \theta+V \sin \theta \cos \varphi) \frac{c_{1}}{r}\right. \\
& \left.-\zeta_{\theta}(U \sin \theta-V \cos \theta \cos \varphi) \frac{c_{1}}{r}-\zeta_{\varphi} V \sin \varphi \frac{c_{1}}{r}\right] \\
& +\varepsilon^{2}\left[\zeta_{r}(U \cos \theta+V \sin \theta \cos \varphi-1)(3(U \cos \theta+V \sin \theta \cos \varphi)+1) \frac{c_{1}}{2 \gamma_{1} k}\right. \\
& -\zeta_{\theta}(U \sin \theta-V \cos \theta \cos \varphi)(U \cos \theta+V \sin \theta \cos \varphi-1) \frac{c_{1}}{\gamma_{1} k} \\
& \left.-\zeta_{\varphi} V \sin \varphi(U \cos \theta+V \sin \theta \cos \varphi-1) \frac{c_{1}}{\gamma_{1} k}+2\left(e_{j} \zeta_{j}\right)^{2}-1\right] \\
& +\cdots, \quad \text { as } \varepsilon \searrow 0 \text { with } \eta / \varepsilon(=r) \text { fixed. }
\end{aligned}
$$

Here, ${ }^{*}$ has been attached to indicate that $\phi_{H}$ is expressed using the inner variable $r$ (or $x_{i}$ ). We observe that the $\varepsilon$-order term coincides with the far-field expansion of $\varepsilon \phi_{F 1}$ for $r \gg 1$ (see (3.52)). Hence, for the inner solution to match the outer solution to order $\varepsilon^{2}$, we should impose the following matching condition:

$$
\begin{aligned}
\phi_{F 2} \rightarrow & \zeta_{r}(U \cos \theta+V \sin \theta \cos \varphi-1)[3(U \cos \theta+V \sin \theta \cos \varphi)+1] \frac{c_{1}}{2 \gamma_{1} k} \\
& -\zeta_{\theta}(U \sin \theta-V \cos \theta \cos \varphi)(U \cos \theta+V \sin \theta \cos \varphi-1) \frac{c_{1}}{\gamma_{1} k} \\
& -\zeta_{\varphi} V \sin \varphi(U \cos \theta+V \sin \theta \cos \varphi-1) \frac{c_{1}}{\gamma_{1} k}+2\left(e_{j} \zeta_{j}\right)^{2}-1, \quad \text { as }|x| \rightarrow \infty
\end{aligned}
$$

In summary, the equation and boundary conditions for $\phi_{F 2}$ are given by

$$
\begin{gathered}
\zeta_{j} \frac{\partial \phi_{F 2}}{\partial x_{j}}=\frac{1}{k} \mathscr{L}\left(\phi_{F 2}\right)+\frac{1}{k} \mathscr{J}\left(\phi_{F 1}, \phi_{F 1}\right), \\
\phi_{F 2}=\mathscr{K}\left(\phi_{F 2}\right)+\left(2 \zeta_{\varphi}^{2}-1\right) S^{2} \sin ^{2} \theta+2 \mathscr{K}\left(\phi_{F 1}\right) \zeta_{\varphi} S \sin \theta, \quad \zeta_{r}>0,|\boldsymbol{x}|=1, \\
\phi_{F 2} \rightarrow \zeta_{r}(U \cos \theta+V \sin \theta \cos \varphi-1)(3(U \cos \theta+V \sin \theta \cos \varphi)+1) \frac{c_{1}}{2 \gamma_{1} k} \\
-\zeta_{\theta}(U \sin \theta-V \cos \theta \cos \varphi)(U \cos \theta+V \sin \theta \cos \varphi-1) \frac{c_{1}}{\gamma_{1} k}
\end{gathered}
$$




\section{S. Taguchi and T. Tsuji}

$$
\begin{aligned}
& -\zeta_{\varphi} V \sin \varphi(U \cos \theta+V \sin \theta \cos \varphi-1) \frac{c_{1}}{\gamma_{1} k} \\
& +2\left(e_{j} \zeta_{j}\right)^{2}-1, \quad \text { as }|\boldsymbol{x}| \rightarrow \infty .
\end{aligned}
$$

Note that $\phi_{F 2}$ depends on the leading-order solution $\phi_{F 1}$ through $\mathscr{J}\left(\phi_{F 1}, \phi_{F 1}\right), \mathscr{K}\left(\phi_{F 1}\right)$ and $c_{1}\left(=-h_{D} / 4 \pi \gamma_{1} k\right)$. In the remaining part, assuming the existence of a solution for the above problem, we derive expressions for the force and torque acting on the sphere.

\subsection{Similarity solutions for the second-order problem and the second-order force and} torque acting on the sphere

We begin by noting the following.

(i) The right-hand side of (3.66) is arranged in the form

$$
(\text { right-hand side })=-\frac{c_{1}}{2 \gamma_{1} k}\left(2 e_{j} \zeta_{j}\right)+(\text { other terms })
$$

Note that the first term is a constant multiple of $\Phi_{\mathrm{U}}^{(1)}$ at infinity (see (3.13) with $J=\mathrm{U})$.

(ii) Since $\phi_{F 1}=\Phi_{\mathrm{U}}^{(1)}+\Phi_{\mathrm{S}}^{(1)}$, the inhomogeneous term in (3.64) is decomposed as

$$
\mathscr{J}\left(\phi_{F 1}, \phi_{F 1}\right)=\mathscr{J}\left(\Phi_{\mathrm{U}}^{(1)}, \Phi_{\mathrm{U}}^{(1)}\right)+\mathscr{J}\left(\Phi_{\mathrm{S}}^{(1)}, \Phi_{\mathrm{S}}^{(1)}\right)+2 \mathscr{J}\left(\Phi_{\mathrm{U}}^{(1)}, \Phi_{\mathrm{S}}^{(1)}\right) .
$$

Then, noting the linearity of the problem, we seek $\phi_{F 2}$ in the form

$$
\phi_{F 2}=-\frac{c_{1}}{2 \gamma_{1} k} \Phi_{\mathrm{U}}^{(1)}+\Phi_{\mathrm{UU}}^{(2)}+\Phi_{\mathrm{SS}}^{(2)}+\Phi_{\mathrm{US}}^{(2)} \text {. }
$$

Here, $\Phi_{\mathrm{UU}}^{(2)}, \Phi_{\mathrm{SS}}^{(2)}$ and $\Phi_{\mathrm{US}}^{(2)}$ solve the following problems:

$$
\begin{aligned}
\zeta_{i} \frac{\partial \Phi_{J}^{(2)}}{\partial x_{i}}= & \frac{1}{k} \mathscr{L}\left(\Phi_{J}^{(2)}\right)+\frac{1}{k} I_{J}^{(2)} \quad(J=\mathrm{UU}, \mathrm{SS}, \mathrm{US}), \\
\Phi_{J}^{(2)}= & \mathscr{K}\left(\Phi_{J}^{(2)}\right)+I_{w, J}^{(2)}, \quad \zeta_{r}>0, r=|\boldsymbol{x}|=1, \\
& \Phi_{J}^{(2)} \rightarrow I_{\infty, J}^{(2)} \quad \text { as } r=|x| \rightarrow \infty
\end{aligned}
$$

where

$$
\begin{aligned}
I_{\mathrm{UU}}^{(2)}= & \mathscr{J}\left(\Phi_{\mathrm{U}}^{(1)}, \Phi_{\mathrm{U}}^{(1)}\right), \quad I_{\mathrm{SS}}^{(2)}=\mathscr{J}\left(\Phi_{\mathrm{S}}^{(1)}, \Phi_{\mathrm{S}}^{(1)}\right), \quad I_{\mathrm{US}}^{(2)}=2 \mathscr{J}\left(\Phi_{\mathrm{U}}^{(1)}, \Phi_{\mathrm{S}}^{(1)}\right), \\
I_{w, \mathrm{UU}}^{(2)}= & 0, \quad I_{w, \mathrm{SS}}^{(2)}=\left(2 \zeta_{\varphi}^{2}-1\right) S^{2} \sin ^{2} \theta, \quad I_{w, \mathrm{US}}^{(2)}=2 \mathscr{K}\left(\left.\Phi_{\mathrm{U}}^{(1)}\right|_{r=1}\right) \zeta_{\varphi} S \sin \theta, \\
I_{\infty, \mathrm{UU}}^{(2)}= & (U \cos \theta+V \sin \theta \cos \varphi)^{2}\left(\frac{3}{2} \frac{c_{1}}{\gamma_{1} k} \zeta_{r}+3 \zeta_{r}^{2}-\zeta^{2}\right) \\
& +(U \cos \theta+V \sin \theta \cos \varphi)\left[(U \sin \theta-V \cos \theta \cos \varphi) \zeta_{\theta}+V \sin \varphi \zeta_{\varphi}\right] \\
& \times\left(-\frac{c_{1}}{\gamma_{1} k}-4 \zeta_{r}\right)+2\left\{\left[(U \sin \theta-V \cos \theta \cos \varphi)^{2}-V^{2} \sin ^{2} \varphi\right] \frac{\zeta_{\theta}^{2}-\zeta_{\varphi}^{2}}{2}\right. \\
& \left.+2 V \sin \varphi(U \sin \theta-V \cos \theta \cos \varphi) \zeta_{\theta} \zeta_{\varphi}\right\}-\frac{c_{1}}{2 \gamma_{1} k} \zeta_{r}+\zeta^{2}-\zeta_{r}^{2}-1, \quad 3.73
\end{aligned}
$$


Inversion of the transverse force on a spinning sphere

$$
I_{\infty, \mathrm{SS}}^{(2)}=I_{\infty, \mathrm{US}}^{(2)}=0 .
$$

Note that $\mathscr{K}\left(\left.\Phi_{\mathrm{S}}^{(1)}\right|_{r=1}\right)=0$ has been used in the second condition of $(3.73 b)$.

Physically, the problem for $\Phi_{\mathrm{UU}}^{(2)}$ (or for $\Phi_{\mathrm{SS}}^{(2)}$ ) describes the second-order Mach number effect in the uniform flow over a sphere (or in the swirling flow around a rotating sphere). On the other hand, the problem for $\varphi_{\text {US }}^{(2)}$ describes the cross effect between the flow past a sphere and the flow induced by a sphere rotation. We call them problems UU, SS and US, respectively. As we see below, only problem US is essential for the transverse force acting on the sphere.

Using the similarity solutions for $\Phi_{U}^{(1)}$ and $\Phi_{S}^{(1)}$ shown in (3.16) and the formulas in Appendix B, we can transform the inhomogeneous term $I_{J}^{(2)}(J=\mathrm{UU}, \mathrm{SS}, \mathrm{US})$ into

$$
\begin{aligned}
& I_{\mathrm{UU}}^{(2)}=(U \cos \theta+V \sin \theta \cos \varphi)^{2} \\
& \times\left[\mathscr{J}_{0}\left(\varphi_{\mathrm{U} a}^{(1)}, \varphi_{\mathrm{U} a}^{(1)}\right)-\frac{\zeta^{2}-\zeta_{r}^{2}}{2} \mathscr{J}_{2}\left(\varphi_{\mathrm{U} b}^{(1)}, \varphi_{\mathrm{U} b}^{(1)}\right)-\mathscr{J}_{3}\left(\varphi_{\mathrm{U} b}^{(1)}, \varphi_{\mathrm{U} b}^{(1)}\right)\right] \\
& +2(U \cos \theta+V \sin \theta \cos \varphi)\left[(U \sin \theta-V \cos \theta \cos \varphi) \zeta_{\theta}+V \sin \varphi \zeta_{\varphi}\right] \mathscr{J}_{1}\left(\varphi_{\mathrm{U} a}^{(1)}, \varphi_{\mathrm{U} b}^{(1)}\right) \\
& +\left\{\left[(U \sin \theta-V \cos \theta \cos \varphi)^{2}-V^{2} \sin ^{2} \varphi\right] \frac{\zeta_{\theta}^{2}-\zeta_{\varphi}^{2}}{2}\right. \\
& \left.+2 V \sin \varphi(U \sin \theta-V \cos \theta \cos \varphi) \zeta_{\theta} \zeta_{\varphi}\right\} \mathscr{J}_{2}\left(\varphi_{\mathrm{U} b}^{(1)}, \varphi_{\mathrm{U} b}^{(1)}\right) \\
& +\frac{\zeta^{2}-\zeta_{r}^{2}}{2} \mathscr{J}_{2}\left(\varphi_{\mathrm{U} b}^{(1)}, \varphi_{\mathrm{U} b}^{(1)}\right)+\mathscr{J}_{3}\left(\varphi_{\mathrm{U} b}^{(1)}, \varphi_{\mathrm{U} b}^{(1)}\right), \\
& I_{\mathrm{SS}}^{(2)}=S^{2} \sin ^{2} \theta\left[\frac{\zeta^{2}-\zeta_{r}^{2}}{2} \mathscr{J}_{2}\left(\varphi_{\mathrm{S}}^{(1)}, \varphi_{\mathrm{S}}^{(1)}\right)+\mathscr{J}_{3}\left(\varphi_{\mathrm{S}}^{(1)}, \varphi_{\mathrm{S}}^{(1)}\right)\right] \\
& -S^{2} \sin ^{2} \theta \frac{\zeta_{\theta}^{2}-\zeta_{\varphi}^{2}}{2} \mathscr{J}_{2}\left(\varphi_{\mathrm{S}}^{(1)}, \varphi_{\mathrm{S}}^{(1)}\right) \\
& I_{\mathrm{US}}^{(2)}=I_{\mathrm{US}}^{(2) \sharp}+I_{\mathrm{US}}^{(2) b}, \\
& I_{\mathrm{US}}^{(2) \sharp}=2 S \sin \theta(U \cos \theta+V \sin \theta \cos \varphi) \zeta_{\varphi} \mathscr{J}_{1}\left(\varphi_{\mathrm{Ua}}^{(1)}, \varphi_{\mathrm{S}}^{(1)}\right) \\
& +2 S \sin \theta\left[-V \sin \varphi \frac{\zeta_{\theta}^{2}-\zeta_{\varphi}^{2}}{2}+(U \sin \theta-V \cos \theta \cos \varphi) \zeta_{\theta} \zeta_{\varphi}\right] \mathscr{J}_{2}\left(\varphi_{\mathrm{U} b}^{(1)}, \varphi_{\mathrm{S}}^{(1)}\right) \\
& +2 S V \sin \theta \sin \varphi\left[\frac{\zeta^{2}-\zeta_{r}^{2}}{2} \mathscr{J}_{2}\left(\varphi_{\mathrm{U} b}^{(1)}, \varphi_{\mathrm{S}}^{(1)}\right)+\mathscr{J}_{3}\left(\varphi_{\mathrm{U} b}^{(1)}, \varphi_{\mathrm{S}}^{(1)}\right)\right] \text {, } \\
& I_{\mathrm{US}}^{(2) b}=2 S \sin \theta(U \sin \theta-V \cos \theta \cos \varphi) \zeta_{r} \mathscr{J}_{4}\left(\varphi_{\mathrm{U} b}^{(1)}, \varphi_{\mathrm{S}}^{(1)}\right) .
\end{aligned}
$$

Here, the operators $\mathscr{J}_{i}, i=0,1,2,3,4$, are defined in Appendix B. Note also that $\mathscr{J}_{i}(\cdot, \cdot)$ appearing above are functions of $r, \zeta_{r}$ and $\zeta$ through $\varphi_{\mathrm{U} a}^{(1)}, \varphi_{\mathrm{U} b}^{(1)}$ and $\varphi_{\mathrm{S}}^{(1)}$. Given these forms, 


\section{S. Taguchi and T. Tsuji}

we consider the following similarity solutions for $\Phi_{\mathrm{UU}}^{(2)}, \Phi_{\mathrm{US}}^{(2)}$ and $\Phi_{\mathrm{SS}}^{(2)}$ (see table 1):

$$
\begin{aligned}
\Phi_{\mathrm{UU}}^{(2)}= & (U \cos \theta+V \sin \theta \cos \varphi)^{2} \varphi_{\mathrm{UU} a}^{(2)}\left(r, \zeta_{r}, \zeta\right) \\
& +(U \cos \theta+V \sin \theta \cos \varphi)\left[(U \sin \theta-V \cos \theta \cos \varphi) \zeta_{\theta}+V \sin \varphi \zeta_{\varphi}\right] \varphi_{\mathrm{UU} b}^{(2)}\left(r, \zeta_{r}, \zeta\right) \\
& +\left[\left((U \sin \theta-V \cos \theta \cos \varphi)^{2}-V^{2} \sin ^{2} \varphi\right) \frac{\zeta_{\theta}^{2}-\zeta_{\varphi}^{2}}{2}\right. \\
& \left.+2 V \sin \varphi(U \sin \theta-V \cos \theta \cos \varphi) \zeta_{\theta} \zeta_{\varphi}\right] \varphi_{\mathrm{UU} c}^{(2)}\left(r, \zeta_{r}, \zeta\right)+\varphi_{\mathrm{UU} d}^{(2)}\left(r, \zeta_{r}, \zeta\right)
\end{aligned}
$$

$$
\begin{gathered}
\Phi_{\mathrm{SS}}^{(2)}=S^{2} \cos ^{2} \theta \varphi_{\mathrm{SS} a}^{(2)}\left(r, \zeta_{r}, \zeta\right)+S^{2} \cos \theta \sin \theta \zeta_{\theta} \varphi_{\mathrm{SS} b}^{(2)}\left(r, \zeta_{r}, \zeta\right) \\
+S^{2} \frac{\zeta_{\theta}^{2}-\zeta_{\varphi}^{2}}{2} \sin ^{2} \theta \varphi_{\mathrm{SS} c}^{(2)}\left(r, \zeta_{r}, \zeta\right)+S^{2} \varphi_{\mathrm{SS} d}^{(2)}\left(r, \zeta_{r}, \zeta\right), \\
\Phi_{\mathrm{US}}^{(2)}=\Phi_{\mathrm{US}}^{(2) \sharp}+\Phi_{\mathrm{US}}^{(2) b}
\end{gathered}
$$

$$
\Phi_{\mathrm{US}}^{(2) \sharp}=S \sin \theta(U \cos \theta+V \sin \theta \cos \varphi) \zeta_{\varphi} \varphi_{\mathrm{US} a}^{(2) \sharp}\left(r, \zeta_{r}, \zeta\right)
$$$$
+S \cos \theta\left[(U \sin \theta-V \cos \theta \cos \varphi) \zeta_{\varphi}-V \sin \varphi \zeta_{\theta}\right] \varphi_{\mathrm{US} b}^{(2) \sharp}\left(r, \zeta_{r}, \zeta\right)
$$$$
+S \sin \theta\left[-V \sin \varphi \frac{\zeta_{\theta}^{2}-\zeta_{\varphi}^{2}}{2}+(U \sin \theta-V \cos \theta \cos \varphi) \zeta_{\theta} \zeta_{\varphi}\right] \varphi_{\mathrm{US} c}^{(2) \sharp}\left(r, \zeta_{r}, \zeta\right)
$$$$
+S V \sin \theta \sin \varphi \varphi_{\mathrm{USd}}^{(2) \sharp}\left(r, \zeta_{r}, \zeta\right),
$$

$$
\begin{aligned}
\Phi_{\mathrm{US}}^{(2) b}= & S \sin \theta(U \sin \theta-V \cos \theta \cos \varphi) \varphi_{\mathrm{US} a}^{(2) b}\left(r, \zeta_{r}, \zeta\right) \\
& +S\left[(U \sin 2 \theta-V \cos 2 \theta \cos \varphi) \zeta_{\theta}+V \cos \theta \sin \varphi \zeta_{\varphi}\right] \varphi_{\mathrm{US} b}^{(2) b}\left(r, \zeta_{r}, \zeta\right) \\
& +S \sin \theta\left[(U \sin \theta-V \cos \theta \cos \varphi) \frac{\zeta_{\theta}^{2}-\zeta_{\varphi}^{2}}{2}+V \sin \varphi \zeta_{\theta} \zeta_{\varphi}\right] \varphi_{\mathrm{US} c}^{(2) b}\left(r, \zeta_{r}, \zeta\right) \\
& +S U \varphi_{\mathrm{US} d}^{(2) b}\left(r, \zeta_{r}, \zeta\right),
\end{aligned}
$$

where $\left(\varphi_{\mathrm{UU} \alpha}^{(2)}, \varphi_{\mathrm{SS} \alpha}^{(2)}, \varphi_{\mathrm{US} \alpha}^{(2) \sharp}, \varphi_{\mathrm{US} \alpha}^{(2) b}\right), \alpha=a, b, c, d$, which are functions of $r, \zeta_{r}$ and $\zeta$, are the solutions to the boundary-value problems shown in Appendix E. Note that they also depend on the parameter $k$.

Because of the similarity solutions, we can obtain the explicit dependency of the second-order macroscopic quantities on $\theta$ and $\varphi$. Below, we only give the results for $P_{r r F 2}$, $P_{r \theta F 2}$ and $P_{r \varphi F 2}$ for conciseness. That is, substituting (3.69) with (3.79)-(3.83) into the definition of $P_{i j F 2}$ (see $(3.4 c)$ ), we obtain

$$
\begin{aligned}
P_{r r F 2}(r, \theta, \varphi)= & -\frac{c_{1}}{2 \gamma_{1} k}(U \cos \theta+V \sin \theta \cos \varphi) \underline{\tilde{P}_{r r, \mathrm{U} a}^{(1)}} \\
& +(U \cos \theta+V \sin \theta \cos \varphi)^{2} \underline{\left(\tilde{P}_{r r}\left[\varphi_{\mathrm{UU} a}^{(2)}\right]-2\left(\tilde{u}_{r, \mathrm{U} a}^{(1)}\right)^{2}\right)}
\end{aligned}
$$


Inversion of the transverse force on a spinning sphere

$$
\begin{aligned}
& +\underline{\tilde{P}_{r r}\left[\varphi_{\mathrm{UUd} d}^{(2)}\right]}+S^{2} \cos ^{2} \theta \underline{\tilde{P}_{r r}\left[\varphi_{\mathrm{SS} a}^{(2)}\right]}+S^{2} \underline{\tilde{P}_{r r}\left[\varphi_{\mathrm{SS} d}^{(2)}\right]} \\
& +S V \sin \theta \sin \varphi \underline{\tilde{P}_{r r}\left[\varphi_{\mathrm{USd}}^{(2) \sharp}\right]}+S \sin \theta(U \sin \theta-V \cos \theta \cos \varphi) \underline{\tilde{P}_{r r}\left[\varphi_{\mathrm{US} a}^{(2) b}\right]} \\
& +S U \tilde{P}_{r r}\left[\varphi_{\mathrm{US} d}^{(2) b}\right], \\
& P_{r \theta F 2}(r, \theta, \varphi)=-\frac{c_{1}}{2 \gamma_{1} k}(U \sin \theta-V \cos \theta \cos \varphi) \underline{\tilde{P}_{r t, \mathrm{U} b}^{(1)}} \\
& +(U \cos \theta+V \sin \theta \cos \varphi)(U \sin \theta-V \cos \theta \cos \varphi) \\
& \times \underline{\left(\tilde{P}_{r t}\left[\varphi_{\mathrm{UU} b}^{(2)}\right]-2 \tilde{u}_{r, \mathrm{U} a}^{(1)} \tilde{u}_{t, \mathrm{U} b}^{(1)}\right)} \\
& +S^{2} \cos \theta \sin \theta \underline{\tilde{P}_{r t}\left[\varphi_{\mathrm{SS} b}^{(2)}\right]}-S V \cos \theta \sin \varphi \underline{\tilde{P}_{r t}\left[\varphi_{\mathrm{US} b}^{(2) \sharp}\right]} \\
& +S(U \sin 2 \theta-V \cos 2 \theta \cos \varphi) \tilde{P}_{r t}\left[\varphi_{\mathrm{US} b}^{(2) b}\right], \\
& P_{r \varphi F 2}(r, \theta, \varphi)=-\frac{c_{1}}{2 \gamma_{1} k} V \sin \varphi \underline{\tilde{P}_{r t, \mathrm{U} b}^{(1)}}+(U \cos \theta+V \sin \theta \cos \varphi) V \sin \varphi \\
& \times \underline{\left(\tilde{P}_{r t}\left[\varphi_{\mathrm{UU} b}^{(2)}\right]-2 \tilde{u}_{r, \mathrm{U} a}^{(1)} \tilde{u}_{t, \mathrm{U} b}^{(1)}\right)} \\
& +S \sin \theta(U \sin \theta+V \sin \theta \cos \varphi)\left(\tilde{P}_{r t}\left[\varphi_{\mathrm{US} a}^{(2) \sharp}\right]-2 \tilde{u}_{r, \mathrm{U} a}^{(1)} \tilde{u}_{t, \mathrm{~S}}^{(1)}\right) \\
& +S \cos \theta(U \sin \theta-V \cos \theta \cos \varphi) \underline{\tilde{P}_{r t}\left[\varphi_{\mathrm{US} b}^{(2) \sharp}\right]} \\
& +S V \cos \theta \sin \varphi \underline{\tilde{P}_{r t}\left[\varphi_{\mathrm{US} b}^{(2) b}\right],}
\end{aligned}
$$

where $\tilde{P}_{r r}[\cdot]$ and $\tilde{P}_{r t}[\cdot]$ are defined in $(3.21 b)$ and the underlined quantities are functions of $r$. Finally, substituting (3.84) into $(3.26 a, b)$ (with $m=2$ ) and carrying out the surface integral, we obtain the second-order force and torque as follows:

$$
\begin{gathered}
\mathcal{F}_{1}^{(2)}=-\frac{c_{1}}{2 \gamma_{1} k} U h_{D}, \quad \mathcal{F}_{2}^{(2)}=-\frac{c_{1}}{2 \gamma_{1} k} V h_{D}, \quad \mathcal{F}_{3}^{(2)}=-S V h_{L}, \\
\mathcal{M}_{1}^{(2)}=\mathcal{M}_{2}^{(2)}=\mathcal{M}_{3}^{(2)}=0,
\end{gathered}
$$

where the dimensionless transverse force $h_{L}$ is defined by

$$
h_{L}=\left.\frac{4}{3} \pi\left(\tilde{P}_{r t}\left[\varphi_{\mathrm{US} a}^{(2) \sharp}\right]-\tilde{P}_{r t}\left[\varphi_{\mathrm{US} b}^{(2) \sharp}\right]+\tilde{P}_{r r}\left[\varphi_{\mathrm{US} d}^{(2) \sharp}\right]\right)\right|_{r=1} .
$$

Note that $h_{L}$ depends on $k$ through $\varphi_{\mathrm{US} a}^{(2) \sharp}, \varphi_{\mathrm{US} b}^{(2) \sharp}$ and $\varphi_{\mathrm{US} d}^{(2) \sharp}$, and hence we write $h_{L}=h_{L}(k)$. Also, it is interesting to note that the transverse force is determined by $\Phi_{\mathrm{US}}^{(2)}$ describing the cross effect between the uniform and rotational flows, in particular by the part $\Phi_{\mathrm{US}}^{(2) \sharp}$.

\subsection{Summary of the force and torque acting on the sphere}

Here, we summarize the expressions of the force and torque acting on the sphere derived from the asymptotic analysis. Let us consider a slightly general situation in which the flow velocity at infinity and the angular velocity of the sphere are given by $\boldsymbol{v}_{\infty}=$ $\left(v_{\infty 1}, v_{\infty 2}, v_{\infty 3}\right)$ and $\boldsymbol{\Omega}_{0}=\left(\Omega_{0,1}, \Omega_{0,2}, \Omega_{0,3}\right)$, respectively. We also define the small parameter $\varepsilon=\left|\boldsymbol{v}_{\infty}\right| /\left(2 R T_{0}\right)^{1 / 2}$ as before, and denote the dimensionless angular velocity 


\section{S. Taguchi and T. Tsuji}

by $S_{i}=L \Omega_{0, i} /\left|\boldsymbol{v}_{\infty}\right|$. Then, the force $p_{0} L^{2} \mathcal{F}_{i}$ and the torque $p_{0} L^{3} \mathcal{M}_{i}$ acting on the sphere are written as

$$
\begin{gathered}
\mathcal{F}_{i}=\varepsilon\left(1+\frac{h_{D}(k)}{8 \pi\left(\gamma_{1} k\right)^{2}} \varepsilon\right) e_{i} h_{D}(k)+\varepsilon^{2}\left(\epsilon_{i j l} e_{j} S_{l}\right) h_{L}(k)+o\left(\varepsilon^{2}\right), \\
\mathcal{M}_{i}=\varepsilon S_{i} h_{M}(k)+o\left(\varepsilon^{2}\right)
\end{gathered}
$$

where $e_{i}=v_{\infty i} /\left|\boldsymbol{v}_{\infty}\right|$ and $h_{D}, h_{L}$ and $h_{M}$ are given by (3.28), (3.86) and (3.29), respectively. In (3.87), we have used (3.50a) to eliminate $c_{1}$. Note also that the functional dependencies of $h_{D}, h_{L}$ and $h_{M}$ on $k$ are explicitly shown in the above formulas.

The formulas (3.87) and (3.88) give the force and torque acting on a rotating sphere as functions of $k$ once the functions $h_{D}(k), h_{M}(k)$ and $h_{L}(k)$ are known. The numerical values of $h_{D}(k)$ were obtained in Takata et al. (1993) (see also Sone 2007), in which the steady flow of a rarefied gas past a sphere (problem $U$ with $U=1$ and $V=0$ ) was investigated based on the linearized Boltzmann equation for a hard-sphere gas under the diffuse reflection boundary condition. Later, Taguchi \& Suzuki (2017) obtained the numerical values of $h_{D}(k)$ for the ellipsoidal statistical model (Holway 1966; Andries et al. 2000; Brull \& Schneider 2008). Concerning $h_{M}$, Taguchi et al. (2019) investigated the steady flow around a rotating sphere (problem $S$ ) based on the ellipsoidal statistical model (and the BGK model), in which the numerical values of $h_{M}(k)$ were obtained for both the BGK and the ellipsoidal statistical model. We also mention that the time-dependent behaviour of a gas caused by the impulsive rotation of a sphere has been recently studied (Taguchi, Tsuji \& Kotera 2021). For readers' convenience, we summarize the values of $h_{D}(k)$ and $h_{M}(k)$ obtained in those studies in tables 3 and 4 , respectively, together with new data obtained in the present study. On the other hand, the function $h_{L}(k)$, which describes the effect of gas rarefaction on the transverse force, is unknown. Therefore, in the next section, we compute $h_{L}(k)$ based on the BGK model.

In table 3 , the values of $h_{D}$ for a hard-sphere gas and for the BGK model are shown against $k$. The viscosity is not adjusted between these models (cf. (3.40a)). If we regard the viscosity given by $(3.40 a)$ as the common basic quantity, we obtain the relation $k^{B G K}=$ $1.2700042427 \times k^{\mathrm{HS}}$. With this conversion, a better agreement of $h_{D}$ between the two models can be obtained (Takata et al. 1993; see also figure $4 a$ below). The drag problem for a rarefied gas has also been investigated by Kalempa \& Sharipov (2020) based on the Shakhov kinetic model. We can derive the values of $h_{D}$ for the Shakhov model from their results.

For subsequent discussions, let us summarize the asymptotic expressions of $h_{D}(k), h_{L}(k)$ and $h_{M}(k)$ for small $k \ll 1$ (for the general molecular model). They are given by

$$
\begin{gathered}
h_{D}=6 \pi \gamma_{1} k\left(1+k_{0} k+O\left(k^{2}\right)\right), \quad k \ll 1, \\
h_{L}=2 \pi\left(1+3 k_{0} k+O\left(k^{2}\right)\right), \quad k \ll 1, \\
h_{M}=-8 \pi \gamma_{1} k\left(1+3 k_{0} k+O\left(k^{2}\right)\right), \quad k \ll 1 .
\end{gathered}
$$

Here, $k_{0}$ represents the slip coefficient (shear slip). The value of $k_{0}$ under the diffuse reflection boundary condition is known as (Sone 2002, 2007)

$$
k_{0}= \begin{cases}-1.25395 & \text { (hard-sphere gas) } \\ -1.01619 & (\text { BGK model })\end{cases}
$$




$\begin{array}{lccccc}k & \text { HS } & \text { BGK } & k & \text { HS } & \text { BGK } \\ 0.009 & - & 0.1681 & 1 & 9.5625 & 8.4168 \\ 0.01 & - & 0.1866 & 1.2 & - & 9.0040 \\ 0.02 & - & 0.3694 & 1.5 & - & 9.6637 \\ 0.03 & - & 0.5485 & 2 & 11.2772 & 10.4056 \\ 0.04 & - & 0.7240 & 3 & - & 11.2381 \\ 0.05 & 1.1091 & 0.8960 & 4 & 12.2333 & 11.6897 \\ 0.06 & - & 1.0645 & 5 & - & 11.9721 \\ 0.07 & - & 1.2297 & 6 & 12.5557 & 12.1645 \\ 0.08 & - & 1.3916 & 7 & - & 12.3040 \\ 0.09 & - & 1.5503 & 8 & - & 12.4096 \\ 0.1 & 2.1168 & 1.7056 & 9 & - & 12.4923 \\ 0.15 & - & 2.4378 & 10 & 12.8071 & 12.5588 \\ 0.2 & 3.8110 & 3.1008 & 12 & - & 12.6590 \\ 0.3 & - & 4.2446 & 15 & - & 12.7598 \\ 0.4 & 6.2292 & 5.1864 & 20 & - & 12.8611 \\ 0.5 & - & 5.9679 & 30 & - & 12.9626 \\ 0.6 & 7.7951 & 6.6228 & 40 & - & 13.0133 \\ 0.7 & - & 7.1781 & 50 & - & 13.0436 \\ 0.8 & - & 7.6522 & \infty & 13.1653 & 13.1653 \\ 0.9 & - & 8.0611 & & & \end{array}$

Table 3. Values of $h_{D}$ for various $k$ for a hard-sphere gas (HS) (Takata et al. 1993) and for the BGK model (Taguchi \& Suzuki 2017) under the diffuse refection boundary condition. Here $k=\infty$ shows the value of $h_{D}$ in the free molecular limit $\left(h_{D}(\infty)=2 \sqrt{\pi}(\pi+8) / 3\right)$. For the BGK model, the values of $h_{D}$ for $k<0.1$ and $k>10$ have been newly obtained in this study.

$\begin{array}{lcrcrc}k & \text { BGK } & k & \text { BGK } & k & \text { BGK } \\ 0.01 & 0.2437 & 0.3 & 3.3047 & 5 & 4.6405 \\ 0.02 & 0.4727 & 0.4 & 3.6197 & 6 & 4.6551 \\ 0.03 & 0.6873 & 0.5 & 3.8271 & 7 & 4.6655 \\ 0.04 & 0.8882 & 0.6 & 3.9723 & 8 & 4.6732 \\ 0.05 & 1.0760 & 0.7 & 4.0790 & 9 & 4.6792 \\ 0.06 & 1.2514 & 0.8 & 4.1602 & 10 & 4.6840 \\ 0.07 & 1.4152 & 0.9 & 4.2239 & 12 & 4.6912 \\ 0.08 & 1.5681 & 1 & 4.2752 & 15 & 4.6983 \\ 0.09 & 1.7108 & 1.5 & 4.4293 & 20 & 4.7054 \\ 0.1 & 1.8441 & 2 & 4.5058 & 50 & 4.7179 \\ 0.15 & 2.3903 & 3 & 4.5813 & \infty & 4.7265 \\ 0.2 & 2.7862 & 4 & 4.6185 & & \end{array}$

Table 4. Values of $-h_{M}$ for various $k$ for the BGK model under the diffuse refection boundary condition (Taguchi et al. 2019). Here $k=\infty$ shows the value of $-h_{M}$ in the free molecular limit $\left(h_{M}(\infty)=-8 \sqrt{\pi} / 3\right)$. The values of $h_{M}$ for $k<0.1$ and $k>10$ have been newly obtained in this study.

The next-order term in the formulas (3.89a) and (3.89c), namely the $k^{2}$-order term in the parentheses, can be found in Sone (2007) for $h_{D}$ and in Taguchi et al. (2019) for $h_{M}$. Note that the expression (3.89b) is new; we give more details on its derivation in $\S 4.3$.

To conclude this section, we present the force and torque using the dimensional quantities initially introduced at the beginning of $\S 2.1$ for a moving sphere. Let $\boldsymbol{F}$ and $M$ denote the force and the moment of force (around the centre) acting on the 


\section{S. Taguchi and T. Tsuji}

sphere, respectively. Then, they are given by

$$
\begin{gathered}
\boldsymbol{F}=-6 \pi \mu_{0} \boldsymbol{L} \boldsymbol{v}_{0}\left(1+\frac{3}{8} \operatorname{Re} \bar{h}_{D}(k)\right) \bar{h}_{D}(k)+\pi \rho_{0} L^{3}\left(\boldsymbol{\Omega}_{0} \times \boldsymbol{v}_{0}\right) \bar{h}_{L}(k), \\
\boldsymbol{M}=-8 \pi \mu_{0} L^{3} \boldsymbol{\Omega}_{0} \bar{h}_{M}(k)(1+o(\operatorname{Re})),
\end{gathered}
$$

where

$$
\bar{h}_{D}(k)=\frac{h_{D}(k)}{6 \pi \gamma_{1} k}, \quad \bar{h}_{L}(k)=\frac{h_{L}(k)}{2 \pi}, \quad \bar{h}_{M}(k)=-\frac{h_{M}(k)}{8 \pi \gamma_{1} k},
$$

and $R e$ is the Reynolds number (see the last paragraph of $\S 2$ ). The $\operatorname{drag} \boldsymbol{F}_{D}$ and the lift $\boldsymbol{F}_{L}$ (transverse force) are therefore written as

$$
\begin{gathered}
\boldsymbol{F}_{D}=-6 \pi \mu_{0} \boldsymbol{L} \boldsymbol{v}_{0}\left(1+\frac{3}{8} \operatorname{Re} \bar{h}_{D}(k)\right) \bar{h}_{D}(k), \\
\boldsymbol{F}_{L}=\pi \rho_{0} L^{3}\left(\boldsymbol{\Omega}_{0} \times \boldsymbol{v}_{0}\right) \bar{h}_{L}(k) .
\end{gathered}
$$

We remark that although the asymptotic analysis has been carried out under the assumption $k=O(1)$, the formulas are applicable to arbitrary $k$ as long as the Reynolds number $R e$ is small. In particular, using the asymptotic expressions (3.89) in (3.94), (3.95), and (3.92) (with $(3.93 a-c))$, we obtain for small $k(=(\sqrt{\pi} / 2) K n)$ the following expressions:

$$
\begin{array}{cc}
\boldsymbol{F}_{D}=-6 \pi \mu_{0} L \boldsymbol{v}_{0}\left(1+\kappa_{0} K n+\frac{3}{8} R e+\cdots\right), & K n \ll 1, \\
\boldsymbol{F}_{L}=\pi \rho_{0} L^{3}\left(\boldsymbol{\Omega}_{0} \times \boldsymbol{v}_{0}\right)\left(1+3 \kappa_{0} K n+\cdots\right), & K n \ll 1, \\
\boldsymbol{M}=-8 \pi \mu_{0} L^{3} \boldsymbol{\Omega}_{0}\left(1+3 \kappa_{0} K n+\cdots\right), & K n \ll 1,
\end{array}
$$

where $\kappa_{0}=(\sqrt{\pi} / 2) k_{0}$ and $K n$ has been used instead of $k\left(\kappa_{0} \approx-1.11128\right.$ for the hard-sphere model and $\kappa_{0} \approx-0.90057$ for the BGK model; see (3.90)).

\section{Computation of the transverse force acting on a sphere}

In this section, we construct $h_{L}(k)$ numerically based on the BGK model. Unlike $h_{D}$ and $h_{M}, h_{L}$ is defined through a second-order problem (see (3.86)). Therefore, the difficulty of computing $h_{L}$ is much more severe than that of computing $h_{D}$ or $h_{M}$. Thus, we are motivated to devise an alternative approach to obtain $h_{L}$, reducing the complexity and making the computation tractable. In this section, we obtain $h_{L}$ along this line. The key is to use a symmetry relation associated with the linearized Boltzmann equation.

Below, we first give some general remarks on the asymptotic analysis based on the BGK model and then derive an alternative formula for $h_{L}$ for the general Boltzmann collision operator (including the BGK operator as a particular case). Finally, we compute $h_{L}$ with the aid of this formula in the case of the BGK collision operator.

\subsection{Remarks on the asymptotic analysis based on the BGK model}

The asymptotic analysis described in $\$ 3$ can be carried out also for the BGK model in a similar way, and essentially the same results are derived. To present the results for the BGK model in a unified way, we expand the operator $\mathscr{J}^{B G K}(\phi)($ see $(2.13))$ as

$$
\mathscr{J}^{B G K}(\phi)=\mathscr{J}^{B G K}(\phi, \phi)+O\left(\phi^{3}\right),
$$


where

$$
\begin{aligned}
\mathscr{J}^{B G K}(\phi, \phi)= & 2\left(\zeta_{i} \zeta_{j}-\frac{\zeta^{2}}{3} \delta_{i j}\right) \tilde{u}_{i}[\phi] \tilde{u}_{j}[\phi]+2 \zeta_{i}\left(\zeta^{2}-\frac{5}{2}\right) \tilde{u}_{i}[\phi] \tilde{\tau}[\phi] \\
& +\frac{1}{2}\left(\zeta^{4}-5 \zeta^{2}+\frac{15}{4}\right) \tilde{\tau}[\phi]^{2}+\tilde{\omega}[\phi] \mathscr{L}^{B G K}(\phi),
\end{aligned}
$$

and $O\left(\phi^{3}\right)$ is the remainder. Here, $\tilde{\omega}[\cdot], \tilde{u}_{i}[\cdot]$ and $\tilde{\tau}[\cdot]$ are defined in $(3.19 a)$. Operator $\mathscr{J}^{B G K}(\phi, \phi)$ is a nonlinear operator and quadratic in $\phi$. We can therefore define the symmetric bilinear form associated with $\mathscr{J}^{\circ} B G K(\phi, \phi)$ by

$$
\begin{aligned}
\mathscr{J}^{B G K}(\psi, \phi)= & \frac{1}{2}\left(\stackrel{\circ}{J}^{B G K}(\psi+\phi, \psi+\phi)-\mathscr{J}^{B G K}(\psi, \psi)-\stackrel{\mathscr{J}}{ }^{B G K}(\phi, \phi)\right) \\
= & \left(\zeta_{i} \zeta_{j}-\frac{\zeta^{2}}{3} \delta_{i j}\right)\left(\tilde{u}_{i}[\psi] \tilde{u}_{j}[\phi]+\tilde{u}_{i}[\phi] \tilde{u}_{j}[\psi]\right) \\
& +\zeta_{i}\left(\zeta^{2}-\frac{5}{2}\right)\left(\tilde{u}_{i}[\psi] \tilde{\tau}[\phi]+\tilde{u}_{i}[\phi] \tilde{\tau}[\psi]\right) \\
& +\frac{1}{2}\left(\zeta^{4}-5 \zeta^{2}+\frac{15}{4}\right) \tilde{\tau}[\psi] \tilde{\tau}[\phi] \\
& +\frac{1}{2}\left(\tilde{\omega}[\psi] \mathscr{L}^{B G K}(\phi)+\tilde{\omega}[\phi] \mathscr{L}^{B G K}(\psi)\right) .
\end{aligned}
$$

Note that $\mathscr{L}^{B G K}(\phi)=2 \mathscr{J}^{B G K}(1, \phi)$ holds (cf. the sentence below (A8)). With these preparations, the results in $\S 3$ apply to the case of the BGK model by taking into account the following correspondence:

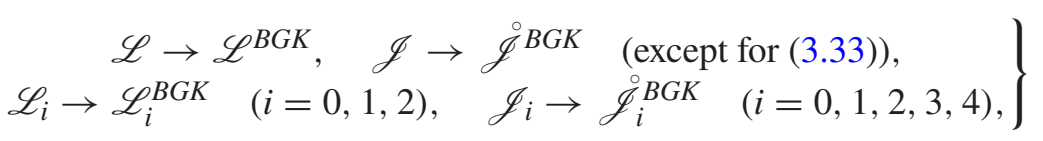

where $\mathscr{L}_{i}^{B G K}$ and $\mathscr{J}_{i}^{B G K}$ are the operators derived from $\mathscr{L}^{B G K}$ and $\mathscr{J}^{B G K}$, whose explicit forms are given in Appendix B (see (B9) and (B10)). Note that in (3.33), the term $\mathscr{J}\left(\phi_{H}, \phi_{H}\right)$ should be replaced by $\mathscr{J}^{B G K}\left(\phi_{H}\right)$.

Since $\mathscr{J}_{4}^{B G K}(\cdot, \cdot)=0$ holds identically (see $(\mathrm{B} 10 e)$ ), the inhomogeneous terms for the problem (E10)-(E12) are absent for the BGK model (see also (E16)). Consequently, the term $\Phi_{\mathrm{US}}^{(2) b}$ (or $\left.\varphi_{\mathrm{US} \alpha}^{(2) b}, \alpha=a, b, c, d\right)$ in $\Phi_{\mathrm{US}}^{(2)}$ happens to be identically zero for this model.

\subsection{Cross-coupling formula}

We now derive an alternative formula for $h_{L}$ for the general collision operator. We can immediately obtain the corresponding result for the BGK model by adopting the corresponding BGK collision operators as shown in (4.4). The following discussion is based on the symmetry relation that relates two problems described by the linearized Boltzmann equation (see, e.g. Sharipov 1994; Takata 2009a,b).

We consider the following auxiliary problem:

(Problem $\mathrm{U}^{\prime}$ ) Consider a sphere (radius $L$ ) at rest with uniform surface temperature $T_{0}$ immersed in a rarefied gas. Let $L x_{i}$ denote Cartesian coordinates whose origin 


\section{S. Taguchi and T. Tsuji}

is at the sphere centre. We denote by $\rho_{0}, T_{0}, p_{0}\left(=\rho_{0} R T_{0}\right)$ and $\boldsymbol{v}_{\infty}^{\prime}$ the (constant) density, temperature, pressure and flow velocity of the gas at infinity and let $\boldsymbol{v}_{\infty}^{\prime}=$ $\left(0,0,\left(2 R T_{0}\right)^{1 / 2} u_{\infty}\right)\left(u_{\infty}>0\right)$. We investigate the steady behaviour of the gas under the same assumptions as (i) and (ii) in $\S 2$ and

(iii)' the flow velocity at infinity $\boldsymbol{v}_{\infty}^{\prime}$ (or $u_{\infty}$ ) is so small that the equation and boundary conditions can be linearized around the reference state at rest with density $\rho_{0}$ and temperature $T_{0}$.

In other words, we consider the same sphere as in the original problem in $\S 2$, but the sphere is at rest, and the flow over it is perpendicular to the $x_{1} x_{2}$ plane.

Let $\rho_{0}\left(2 R T_{0}\right)^{-3 / 2}\left(1+u_{\infty} \Phi_{\mathrm{U} *}^{(1)}(\boldsymbol{x}, \zeta)\right) E$ denote the velocity distribution function of the gas molecules in problem $U^{\prime}$. Then, using the same notation as before, $\Phi_{\mathrm{U} *}^{(1)}$ satisfies the following equation and boundary conditions:

$$
\begin{gathered}
\zeta_{i} \frac{\partial \Phi_{\mathrm{U} *}^{(1)}}{\partial x_{i}}=\frac{1}{k} \mathscr{L}\left(\Phi_{\mathrm{U} *}^{(1)}\right), \quad|\boldsymbol{x}|>1, \\
\Phi_{\mathrm{U} *}^{(1)}=\mathscr{K}\left(\Phi_{\mathrm{U} *}^{(1)}\right), \quad \zeta_{r}>0, \quad|\boldsymbol{x}|=1, \\
\Phi_{\mathrm{U} *}^{(1)} \rightarrow 2 \zeta_{3} \quad \text { as }|\boldsymbol{x}| \rightarrow \infty .
\end{gathered}
$$

Note that by assumption (i), we implicitly assume that the molecular model is common for problem $\mathrm{U}^{\prime}$ and the original one in $\S 2$.

The problem $U^{\prime}$ is essentially problem $U$ that arose in $\$ 3.2$ (see (3.11)-(3.13) with $J=$ $\mathrm{U})$, but there is a difference in the flow direction at infinity. We chose this flow direction to be consistent with the direction of the transverse force in the original problem. Moreover, the equivalence between problem $\mathrm{U}^{\prime}$ and problem $U$ suggests that we can write $\Phi_{\mathrm{U}}^{(1)}$ in terms of the similarity solutions. Indeed, straightforward computations show that $\Phi_{\mathrm{U} *}^{(1)}$ is given by

$$
\Phi_{\mathrm{U} *}^{(1)}=(\sin \theta \sin \varphi) \varphi_{\mathrm{U} a}^{(1)}\left(r, \zeta_{r}, \zeta\right)-\left(\zeta_{\theta} \cos \theta \sin \varphi+\zeta_{\varphi} \cos \varphi\right) \varphi_{\mathrm{U} b}^{(1)}\left(r, \zeta_{r}, \zeta\right),
$$

where $\varphi_{\mathrm{U} a}^{(1)}$ and $\varphi_{\mathrm{U} b}^{(1)}$ are the solution to (3.17). Therefore, if we know the solution to problem $\mathrm{U}$, we essentially know the solution to problem $\mathrm{U}^{\prime}$.

We now return to the boundary-value problem for $\Phi_{\mathrm{US}}^{(2)}$ arising in the second-order problem in the asymptotic analysis, that is, (3.70)-(3.72) with $J=$ US. The equations for $\Phi_{\mathrm{U} *}^{(1)}$ and $\Phi_{\mathrm{US}}^{(2)}$ are both linearized Boltzmann equations, and the application of the symmetry relation described in Takata (2009a) (Proposition 2 there) leads to the following identity, valid for the entire range of $k(0<k<\infty)$ :

$$
S V h_{L}=\int_{|x|=1}\left\langle\zeta_{r} \Phi_{\mathrm{U} *}^{(1)} I_{w, \mathrm{US}}^{(2)-}\right\rangle \mathrm{d} S-\frac{1}{k} \int_{|x|>1}\left\langle\Phi_{\mathrm{U} *}^{(1)} I_{\mathrm{US}}^{(2)-}\right\rangle \mathrm{d} \boldsymbol{x}
$$

where $I_{w, \mathrm{US}}^{(2)}$ and $I_{\mathrm{US}}^{(2)}$ are given in (3.73b) and (3.73a), $\mathrm{d} \boldsymbol{x}=\mathrm{d} x_{1} \mathrm{~d} x_{2} \mathrm{~d} x_{3}$ and ${ }^{-}$indicates the reflection operator acting on the variable $\zeta_{i}$, that is, $F^{-}\left(\zeta_{i}\right)=F\left(-\zeta_{i}\right)$ for any function $F\left(\zeta_{i}\right)$. The range of the first (second) integral is the whole surface of the sphere (the whole gas region). Note that $I_{w, \mathrm{US}}^{(2)}$ and $I_{\mathrm{US}}^{(2)}$ are determined by $\Phi_{\mathrm{U}}^{(1)}$ and $\Phi_{\mathrm{S}}^{(1)}$. Therefore, if we know $\Phi_{\mathrm{U}}^{(1)}$ and $\Phi_{\mathrm{S}}^{(1)}$ (and thus $\Phi_{\mathrm{U} *}^{(1)}$ ), the above identity gives a closed expression for $h_{L}$, 


\section{Inversion of the transverse force on a spinning sphere}

which can be evaluated without knowledge of $\Phi_{\mathrm{US}}^{(2)}$. Moreover, substituting (3.16) and (4.6) and integrating the result with respect to $\theta$ and $\varphi$, we can further simplify (4.7) to

$$
h_{L}=\frac{4}{3} \pi\left[I_{w}-\frac{1}{k}\left(I_{1}+I_{2}\right)\right] \text {, }
$$

where

$$
\begin{gathered}
I_{w}=\mathscr{K}\left(\left.\varphi_{\mathrm{U} a}^{(1)}\right|_{r=1}\right) \tilde{P}_{r t, \mathrm{U} b}^{(1)}(r=1), \\
I_{1}=2 \int_{1}^{\infty} r^{2}\left\langle\varphi_{\mathrm{U} a}^{(1)}\left[\frac{\zeta^{2}-\zeta_{r}^{2}}{2} \mathscr{J}_{2}\left(\varphi_{\mathrm{U} b}^{(1)}, \varphi_{\mathrm{S}}^{(1)}\right)^{-}+\mathscr{J}_{3}\left(\varphi_{\mathrm{U} b}^{(1)}, \varphi_{\mathrm{S}}^{(1)}\right)^{-}\right]\right\rangle \mathrm{d} r, \\
I_{2}=2 \int_{1}^{\infty} r^{2}\left\langle\frac{\zeta^{2}-\zeta_{r}^{2}}{2} \varphi_{\mathrm{U} b}^{(1)} \mathscr{J}_{1}\left(\varphi_{\mathrm{U} a}^{(1)}, \varphi_{\mathrm{S}}^{(1)}\right)^{-}\right\rangle \mathrm{d} r .
\end{gathered}
$$

Here, $\mathscr{J}_{l}(\cdot, \cdot)^{-}\left(r, \zeta_{r}, \zeta\right)=\mathscr{J}_{l}(\cdot, \cdot)\left(r,-\zeta_{r}, \zeta\right), l=1,2,3$. In this formula, $h_{L}$ is expressed in terms of $\left(\varphi_{\mathrm{U} a}^{(1)}, \varphi_{\mathrm{U} b}^{(1)}\right)$ and $\varphi_{\mathrm{S}}^{(1)}$ only, namely the solutions of the elementary problems $\mathrm{U}$ and $\mathrm{S}$. In this way, we can bypass the difficulty to solve the second-order problem as far as $h_{L}$ is concerned.

The formula (4.8) (or (4.7)) underlines that the transverse force is a cross effect between the translational and swirling motion. In this sense, we may refer to (4.8) as a cross-coupling formula. Sharipov (2011) discussed a similar expression for a general weakly perturbed Boltzmann system. In this connection, we stress that, although the transverse force in the present problem is of second order in $\varepsilon$, it is a leading-order effect since the first-order transverse force degenerates. We also remark that the asymptotic properties of $\Phi_{\mathrm{U} *}^{(1)}$ and $\Phi_{\mathrm{US}}^{(2)}$ as $|\boldsymbol{x}| \rightarrow \infty$ play a crucial role in deriving the formula (4.7), as discussed in Takata $(2009 a)$. To see this point more clearly, we give a direct derivation of the formula in Appendix F.

\subsection{The function $h_{L}(k)$ for $k \rightarrow \infty$ and for $k \ll 1$}

As an application of the formula (4.8), let us first consider two limiting cases, the case of $k \rightarrow \infty$ and the case of $k \ll 1$.

First, we explain the case of $k \rightarrow \infty$ (i.e. the free molecular gas or collisionless gas). In this case, the term $k^{-1}\left(I_{1}+I_{2}\right)$ is negligibly small compared to $I_{w}$ and therefore is ignored. On the other hand, the term $I_{w}$ can be easily calculated with the aid of the free molecular solution for problem $\mathrm{U}$ (see Appendix $\mathrm{G}$ for a summary of the free molecular solution). Since $\mathscr{K}\left(\left.\varphi_{\mathrm{U} a}^{(1)}\right|_{r=1}\right)=-\sqrt{\pi}$ and $\tilde{P}_{r t, \mathrm{U} b}^{(1)}(r=1)=1 / \sqrt{\pi}(\mathrm{cf} .(\mathrm{G} 3 e))$, we obtain

$$
h_{L} \rightarrow-\frac{4}{3} \pi \quad \text { as } k \rightarrow \infty \quad \text { (collisionless gas). }
$$

Meanwhile, we can also compute $h_{L}$ directly from (3.86) using the second-order macroscopic quantities for the free molecular flow. According to $(\mathrm{G} 6 e),(\mathrm{G} 7 c)$ and $(\mathrm{G} 7 e)$, we have $\tilde{P}_{r r}\left[\varphi_{\mathrm{US} d}^{(2) \sharp}\right](r=1)=0, \tilde{P}_{r t}\left[\varphi_{\mathrm{US} b}^{(2) \sharp}\right](r=1)=0$ and $\tilde{P}_{r t}\left[\varphi_{\mathrm{US} a}^{(2) \sharp}\right](r=1)=-1$. Therefore, (3.86) gives $h_{L}=-(4 / 3) \pi$, which coincides with (4.10).

Equation (4.10) implies that a sphere moving in a collisionless gas with translational and angular velocities $\boldsymbol{v}_{0}$ and $\boldsymbol{\Omega}_{0}$ is subject to the (dimensional) transverse force $-\frac{2}{3} \pi \rho_{0} L^{3}\left(\boldsymbol{\Omega}_{0} \times \boldsymbol{v}_{0}\right)$. The direction of the force is therefore opposite to the corresponding force in the continuum flow (e.g. Rubinow \& Keller 1961). This result, pointed out by Wang (1972), Ivanov \& Yanshin (1980) and Borg et al. (2003), is known as the inverse 


\section{S. Taguchi and T. Tsuji}

Magnus effect. It may be worth noting that Ivanov \& Yanshin (1980) arrived at this conclusion without the smallness assumptions for $\boldsymbol{\Omega}_{0}$ and $\boldsymbol{v}_{0}$.

Next, we consider the case of $k \ll 1$. To explicitly evaluate the terms $I_{w}, I_{1}$ and $I_{2}$ in (4.8), we need the explicit forms of the velocity distribution function for problems $U$ and $\mathrm{S}$ (i.e. $\varphi_{\mathrm{U} a}^{(1)}, \varphi_{\mathrm{U} b}^{(1)}$ and $\left.\varphi_{\mathrm{S}}^{(1)}\right)$ for small $k$. Such expressions can be obtained with the aid of the asymptotic theory of the Boltzmann equation with small Knudsen numbers (Sone 2002, 2007). More precisely, we first apply the theory to the system (3.11)-(3.13) to obtain asymptotic expressions for $\varphi_{\mathrm{U} a}^{(1)}, \varphi_{\mathrm{U} b}^{(1)}$ and $\varphi_{\mathrm{S}}^{(1)}$ for small $k$. Then, we substitute them into (4.9) to compute $I_{w}, I_{1}$ and $I_{2}$ explicitly.

In this study, we intend to derive an expression for $h_{L}(k)$ for small $k$ up to order $k$. This means that we need to obtain $I_{1}$ and $I_{2}$ (or $I_{w}$ ) to order $k^{2}$ (or $k$ ). The derivation is straightforward but lengthy. Therefore, we omit it here and only give the final results. That is, $I_{w}, I_{1}$ and $I_{2}$ are expressed for small $k$ as

$$
\begin{gathered}
I_{w}=-\frac{9}{4} \gamma_{1}^{2} k^{2}+O\left(k^{3}\right), \quad k \ll 1, \\
I_{1}=-\frac{11}{8} k\left(1+3 k_{0} k+O\left(k^{2}\right)\right), \quad k \ll 1, \\
I_{2}=-\frac{1}{8} k\left(1+3 k_{0} k+O\left(k^{2}\right)\right), \quad k \ll 1 .
\end{gathered}
$$

Substituting them into (4.8), we obtain (3.89b). Note that the leading-order term of $(3.89 b)$ coincides with the result obtained by Rubinow \& Keller (1961) based on the incompressible Navier-Stokes equation (with no-slip boundary conditions). Since $k_{0}$ is negative (see (3.90)), the magnitude of the transverse force decreases with an increase of $k$ when $k \ll 1$.

\section{4. $h_{L}(k)$ for intermediate values of $k$}

In this subsection, we compute the function $h_{L}(k)$ numerically utilizing the formula (4.8). We employ the BGK model of the Boltzmann equation to reduce the complexity in the numerical integration. We thus replace the operators $\mathscr{J}_{i}(i=1,2,3)$ in $I_{1}$ and $I_{2}$ with $\mathscr{J}_{i}^{B G K}$ (see Appendix B for their explicit forms). Consequently, many integrals with respect to the molecular velocity can be carried out, and we obtain for the BGK model the following:

$$
\begin{gathered}
I_{1}=-\int_{1}^{\infty} r^{2} \tilde{u}_{t, \mathrm{U} b}^{(1)} \tilde{u}_{t, \mathrm{~S}}^{(1)}\left(\tilde{P}_{r r, \mathrm{U} a}^{(1)}-\tilde{P}_{\mathrm{U} a}^{(1)}\right) \mathrm{d} r \\
I_{2}=2 \int_{1}^{\infty} r^{2} \tilde{u}_{t, \mathrm{~S}}^{(1)}\left(-\tilde{u}_{r, \mathrm{U} a}^{(1)} \tilde{P}_{r t, \mathrm{U} b}^{(1)}+\tilde{\tau}_{\mathrm{U} a}^{(1)} \tilde{Q}_{t, \mathrm{U} b}^{(1)}\right) \mathrm{d} r \\
+2 \int_{1}^{\infty} r^{2} \tilde{\omega}_{\mathrm{U} a}^{(1)}\left[\tilde{u}_{t, \mathrm{U} b}^{(1)} \tilde{u}_{t, \mathrm{~S}}^{(1)}\right. \\
\left.-\pi \int_{0}^{\infty} \int_{0}^{\pi} \zeta^{4} \sin ^{3} \theta_{\zeta} \varphi_{\mathrm{U} b}^{(1)}\left(r, \theta_{\zeta}, \zeta\right) \varphi_{\mathrm{S}}^{(1)}\left(r, \pi-\theta_{\zeta}, \zeta\right) E \mathrm{~d} \theta_{\zeta} \mathrm{d} \zeta\right] \mathrm{d} r .
\end{gathered}
$$

Here, $\tilde{u}_{t, \mathrm{U} b}^{(1)}(r), \tilde{u}_{t, \mathrm{~S}}^{(1)}(r), \tilde{P}_{r r, \mathrm{U} a}^{(1)}(r), \tilde{P}_{\mathrm{U} a}^{(1)}(r), \tilde{u}_{r, \mathrm{U} a}^{(1)}(r), \tilde{P}_{r t, \mathrm{U} b}^{(1)}(r), \tilde{\tau}_{\mathrm{U} a}^{(1)}(r), \tilde{Q}_{t, \mathrm{U} b}^{(1)}(r)$ and $\tilde{\omega}_{\mathrm{U} a}^{(1)}(r)$, defined in (3.23), are the macroscopic variables arising in problems $U$ and $S$. In the last term of $I_{2}$, the variable $\theta_{\zeta}=\cos ^{-1}\left(\zeta_{r} / \zeta\right)\left(0 \leq \theta_{\zeta} \leq \pi\right)$ is used in place of $\zeta_{r}$. Accordingly, $\varphi_{\mathrm{U} b}^{(1)}$ and $\varphi_{\mathrm{S}}^{(1)}$ are regarded as functions of $r, \theta_{\zeta}$ and $\zeta$. Note that the integral with respect to $\theta_{\zeta}$ is a convolution of $\varphi_{\mathrm{U} b}^{(1)}$ and $\varphi_{\mathrm{S}}^{(1)}$. 


\section{Inversion of the transverse force on a spinning sphere}

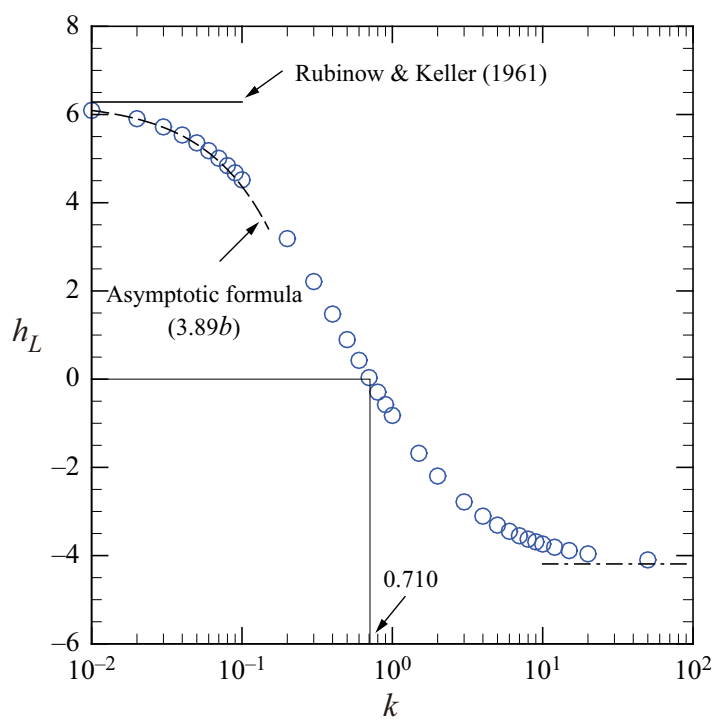

Figure 3. Plot of $h_{L}$ versus $k$ for the BGK model under the diffuse reflection boundary condition. The circle symbols represent the numerical results. The asymptotic formula (3.89b) with $k_{0}=-1.01619$ (see (3.90)) is shown by the dashed curve. The asymptotic values at $k \rightarrow 0$ (the continuum limit) and $k \rightarrow \infty$ (the free molecular limit) are shown by the solid and dash-dotted lines, respectively. The value of $h_{L}$ decreases with $k$ and intersects $h_{L}=0$ at $k=k_{t h} \approx 0.710$.

The numerical solutions of $\left(\varphi_{\mathrm{U} a}^{(1)}, \varphi_{\mathrm{U} b}^{(1)}\right)$ and $\varphi_{\mathrm{S}}^{(1)}$ based on the BGK model have been prepared beforehand in Taguchi \& Suzuki (2017) for problem U and in Taguchi et al. (2019) for problem S, using an accurate finite difference method (Takata et al. 1993). Therefore, we can make use of these data to carry out the numerical integration in (4.12). We also made additional computations to supplement the data, in particular for $k<0.1$ and $k>10$ (see also the second paragraph of $\S 3.7$ ). See Appendix H for further details of the numerical computations.

Figure 3 shows the obtained $h_{L}$ as a function of $k$. In the figure, the symbols represent the numerical results, the values of which are also tabulated in table 5. As seen from the figure and table, $h_{L}$ decreases monotonically with $k$. The dashed curve shows the asymptotic formula $(3.89 b)$ for small $k$, and the solid (horizontal) line represents its leading-order term $\left(h_{L}=2 \pi\right)$, corresponding to the Navier-Stokes result (Rubinow \& Keller 1961). The dash-dotted line shows the result for the free molecular gas (see (4.10)). The numerical results tend to approach both $2 \pi$ and $-\frac{4}{3} \pi$ as $k \rightarrow 0$ and $k \rightarrow \infty$, respectively. The values of $h_{L}$ in these limits have different signs, and the curve $h_{L}(k)$ intersects $h_{L}=0$ at an intermediate value of $k$. Writing this value $k_{t h}$ (i.e. the threshold of $k$ above which the negative lift occurs), we find $k_{t h}=0.710$ for the present computation based on the BGK model and the diffuse reflection boundary condition. In this way, the present asymptotic theory supplemented by the numerical computations for $h_{L}$ can describe the change of the transverse force in terms of the Knudsen number and, in particular, the transition to negative lift.

In order to obtain further insight into the present results, we compare the formulas (3.87) and (3.88) with existing numerical results, in particular with those obtained by the DSMC method (Volkov 2011). To this end, we consider the case in which the angular velocity of the sphere $\boldsymbol{\Omega}_{0}$ is perpendicular to the flow velocity at infinity $\boldsymbol{v}_{\infty}$, i.e. $\boldsymbol{\Omega}_{0}=\left(\Omega_{0}, 0,0\right)$ and 


\section{S. Taguchi and T. Tsuji}

\begin{tabular}{lllllc}
$k$ & \multicolumn{1}{c}{$h_{L}$} & $k$ & \multicolumn{1}{c}{$h_{L}$} & $k$ & $h_{L}$ \\
0.01 & $6.0935(6.0916)^{a}$ & 0.3 & 2.2129 & 4 & -3.1052 \\
0.02 & $5.9047(5.9001)^{a}$ & 0.4 & 1.4763 & 5 & -3.3085 \\
0.03 & $5.7189(5.7085)^{a}$ & 0.5 & 0.8970 & 6 & -3.4481 \\
0.04 & $5.5358(5.5170)^{a}$ & 0.6 & 0.4272 & 7 & -3.5498 \\
0.05 & $5.3563(5.3254)^{a}$ & 0.7 & 0.0362 & 8 & -3.6271 \\
0.06 & $5.1806(5.1339)^{a}$ & 0.8 & -0.2935 & 9 & -3.6879 \\
0.07 & $5.0089(4.9424)^{a}$ & 0.9 & -0.5762 & 10 & -3.7368 \\
0.08 & $4.8417(4.7508)^{a}$ & 1 & -0.8213 & 12 & -3.8108 \\
0.09 & $4.6789(4.5593)^{a}$ & 1.5 & -1.6807 & 15 & -3.8855 \\
0.1 & $4.5204(4.3677)^{a}$ & 2 & -2.1964 & 20 & -3.9607 \\
0.2 & 3.1866 & 3 & -2.7827 & 50 & -4.0972
\end{tabular}

Table 5. Values of $h_{L}$ for various $k$ for the BGK model under the diffuse refection boundary condition. Note that $h_{L} \rightarrow-\frac{4}{3} \pi \approx-4.1888$ as $k \rightarrow \infty$. ${ }^{a}$ The results obtained by using the asymptotic formula $(3.89 b)$.

$\boldsymbol{v}_{\infty}=\left(0, v_{\infty}, 0\right)$ with $\Omega_{0}>0$ and $v_{\infty}>0$ (or $\left.\alpha_{0}=\pi / 2\right)$ in figure 1. Following Volkov (2011), we introduce the drag, lift and torque coefficients by

$$
C_{D}=\frac{F_{D}}{\frac{1}{2} \rho_{0} \pi L^{2} v_{\infty}^{2}}, \quad C_{L}=\frac{-F_{L}}{\frac{1}{2} \rho_{0} \pi L^{3} v_{\infty} \Omega_{0}}, \quad C_{T}=\frac{-M}{\frac{1}{2} \rho_{0} \pi L^{5} \Omega_{0}^{2}},
$$

where $\left(0, F_{D}, 0\right),\left(0,0, F_{L}\right)$ and $(M, 0,0)$ are the drag, lift and moment of force (torque) acting on the sphere. Note that these coefficients depend on physical parameters $K n=$ $\ell_{0} / L, M a=v_{\infty} /\left(5 R T_{0} / 3\right)^{1 / 2}$ and $\hat{\Omega}_{0}=L \Omega_{0} /\left(2 R T_{0}\right)^{1 / 2}$. If we use the expressions of the forces and torque for small $M a \ll 1$ obtained in the present study, $C_{D}, C_{L}$ and $C_{T}$ are given, at leading order in $M a$, as

$$
\begin{gathered}
\sqrt{\frac{5}{6}} M a C_{D}=\frac{h_{D}}{\pi}, \\
C_{L}=\frac{h_{L}}{\pi}, \\
\hat{\Omega}_{0} C_{T}=-\frac{h_{M}}{\pi} .
\end{gathered}
$$

In figure 4, we compare $C_{D}, C_{L}$ and $C_{T}$ based on (4.14) with those obtained by Volkov (2011) for various $K n$ and $M a$ in the case of $\hat{\Omega}_{0}=0.1$. Here, to evaluate $C_{D}, C_{L}$ and $C_{T}$ from (4.14), we have used the numerical data of $h_{D}, h_{L}$ and $h_{M}$ for the BGK model shown in tables 3, 5 and 4. For $k<0.01$, we have used the asymptotic formulas (3.89a)-(3.89c). Note that the DSMC results are for a hard-sphere gas, but the data $h_{D}, h_{L}$ and $h_{M}$ are for the BGK model. For the drag, it is known that the agreement between the BGK model and the hard-sphere model is enhanced if we make a conversion of the Knudsen number (see the third paragraph of $\S 3.7)$. This conversion has been used in figure $4(a)$, that is, $C_{D}$ for the BGK model is shown against $K n / 1.270 \cdots$. We also mention that the original values of the Knudsen number presented in Volkov (2011) (e.g. figure 8 there) have been multiplied by $\sqrt{\pi}$ in figure 4(a-c); otherwise, the values of $C_{D}$ by Volkov (2011) deviate systematically from the known results based on the linearized Boltzmann equation for a hard-sphere gas (Takata et al. 1993), resulting in an inconsistency. As seen from figure 4(b), the agreement of $C_{L}$ between the present results and the DSMC results is quite encouraging. 

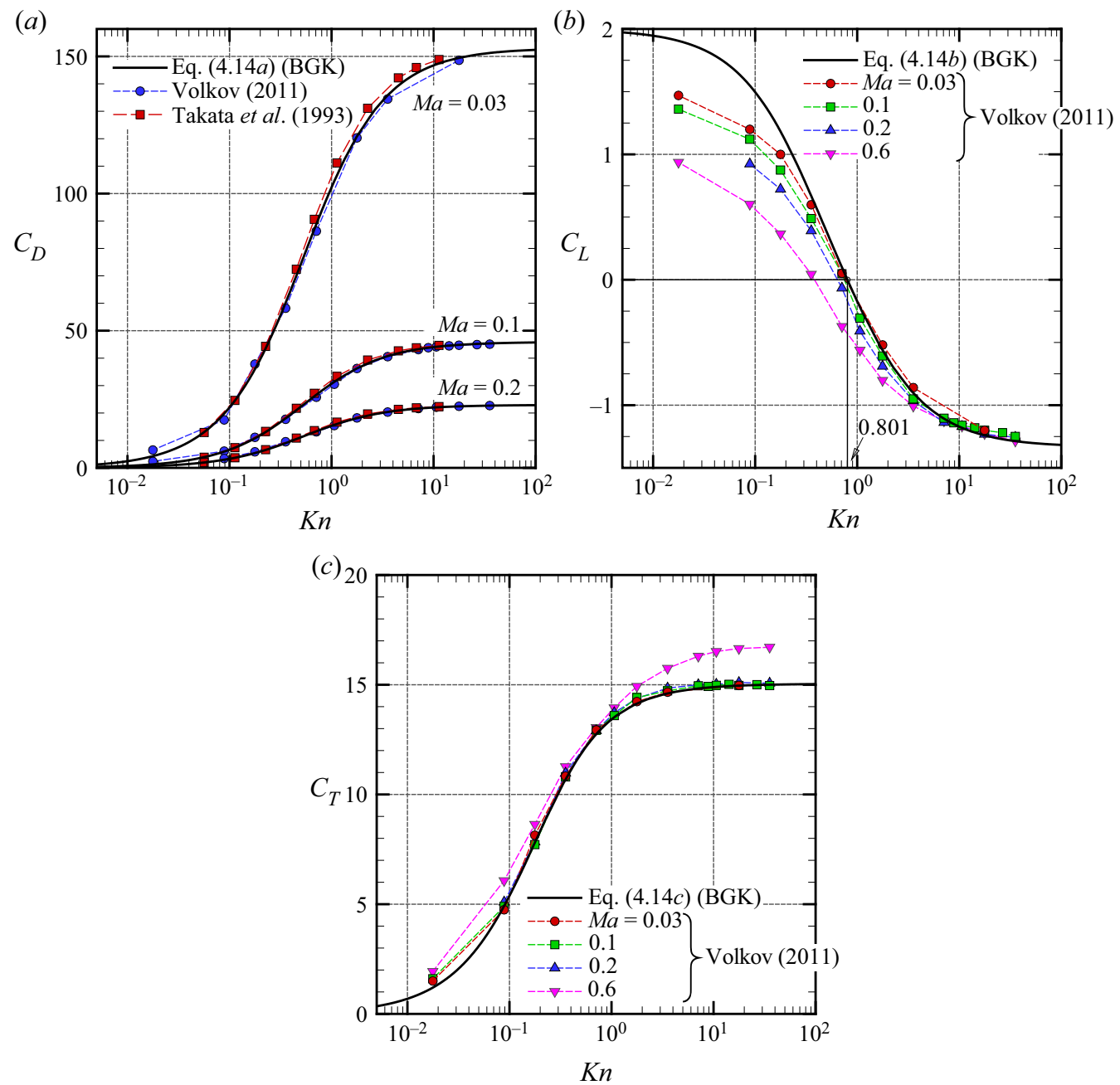

Figure 4. Coefficients (a) $C_{D}$, (b) $C_{L}$ and (c) $C_{T}$ as a function of $K n$ in the case of $L \Omega_{0} /\left(2 R T_{0}\right)^{1 / 2}=0.1$. The solid lines represent (4.14) using the data of $h_{D}, h_{L}$ and $h_{M}$ for the BGK model. The symbols show the results of the DSMC computations for a hard-sphere gas (Volkov 2011). The results for $C_{D}$ based on the linearized Boltzmann equation for a hard-sphere gas (Takata et al. 1993) (i.e. (4.14a) using the data of $h_{D}$ for hard-sphere gas in table 3) are also shown in (a).

When $M a=0.03, C_{L}$ obtained by the present formula (the solid curve) and $C_{L}$ by the DSMC data (the red circle) are close each other for $K n \gtrsim 0.4$. In particular, the Knudsen number at which $C_{L}$ vanishes in the DSMC simulations seems to be close to the point $K n=K n_{t h} \approx 0.801$ obtained by the present theory, despite the difference in the molecular model. As the Mach number increases, the discrepancy between the DSMC and present results becomes larger. We also notice that the discrepancy is more pronounced for smaller $K n$. We recall that the Mach number should be smaller than $K n$ for the present theory to be applicable (the Reynolds number should be small). Therefore, the limit $K n \rightarrow 0$ with $M a$ fixed does not correspond to our analysis, which explains the observed discrepancy for small $K n$. The torque coefficient obtained through $h_{M}$ also compares favourably with the DSMC results when $M a \leq 0.2$ (see figure $4 c$ ). 


\section{S. Taguchi and T. Tsuji}

\section{Concluding remarks}

In this paper, we considered the flow around a spinning sphere moving in a rarefied gas. In this situation, the sphere is subject to a transverse force (lift force) in addition to a drag force. Previous studies suggest that the transverse force acting on the sphere changes direction with an increase of the Knudsen number. This study aimed to reveal precisely the transition of the transverse force with the Knudsen number, assuming that the relative speed between the sphere and the surrounding gas is small (weakly nonlinear setting).

The present analysis is based on the method of matched asymptotic expansions for the Boltzmann equation for small Mach numbers. The physical space is divided into two regions based on the solution's underlying characteristic length. Then, we constructed the asymptotic solution in each region by considering their matching in the crossover region. As a result, we successfully derived the boundary-value problems of the linearized Boltzmann equation for the near region up to the second order of Mach numbers. Then, with the aid of the similarity solutions, we derived the expressions of the force and torque acting on the sphere, which are summarized in (3.87) and (3.88) (or (3.91) and (3.92)).

The second outcome of this study is the actual construction of the force formula. In the derived expressions, the force and torque exerted on the sphere depend on the Knudsen number through the functions $h_{D}(k), h_{L}(k)$ and $h_{M}(k)$, and their numerical data are necessary to complete the formulas. The numerical values of $h_{D}(k)$ and $h_{M}(k)$ are available in the existing literature, but $h_{L}$ is new. In this study, we obtained $h_{L}(k)$ (the transverse force) numerically based on the BGK model of the Boltzmann equation for a wide range of $k$ (we also derived its asymptotic expression for small $k$ for the general collision model). The obtained $h_{L}$ exhibits a monotonic decrease in $k$ and changes sign when crossing the threshold $k_{t h} \approx 0.710$. Thus, the transverse force acting on the sphere reverses its direction when the Knudsen number is above this threshold for any sphere velocity $\boldsymbol{v}_{0}$ and rotation $\boldsymbol{\Omega}_{0}$ in the present weakly perturbed system.

Wang (1972), Ivanov \& Yanshin (1980) and Borg et al. (2003) pointed out that the direction of the transverse force is opposite in the free molecular gas with respect to that for the continuum flow. The present work connects these two limits and clarifies the transition of the transverse force in terms of the Knudsen number.

Acknowledgements. A part of the numerical computations was carried out using the supercomputer of ACCMS, Kyoto University. We thank Professor A.N. Volkov for providing numerical data for comparison.

Funding. This work was supported by JSPS KAKENHI grant nos. 25820041 and $20 \mathrm{H} 02067$.

Declaration of interests. The authors report no conflict of interest.

Author ORCIDs.

(1) Satoshi Taguchi https://orcid.org/0000-0002-0661-7058;

(ㄱ) Tetsuro Tsuji https://orcid.org/0000-0002-2087-5459.

\section{Appendix A. The Boltzmann equation and the BGK model}

Using the quantities introduced at the beginning of $\S 2.2$, the time-independent Boltzmann equation is written as

$$
\begin{gathered}
\xi_{i} \frac{\partial f}{\partial X_{i}}=J(f, f), \\
J(f, f)=\frac{1}{m} \int\left(f\left(\xi^{\prime}\right) f\left(\boldsymbol{\xi}_{*}^{\prime}\right)-f(\boldsymbol{\xi}) f\left(\boldsymbol{\xi}_{*}\right)\right) \mathcal{B}\left(\frac{|\boldsymbol{\alpha} \cdot \boldsymbol{V}|}{|V|},|V|\right) \mathrm{d} \Omega(\boldsymbol{\alpha}) \mathrm{d} \boldsymbol{\xi}_{*},
\end{gathered}
$$


Inversion of the transverse force on a spinning sphere

$$
\xi^{\prime}=\xi+\left[\left(\xi_{*}-\xi\right) \cdot \alpha\right] \alpha, \quad \xi_{*}^{\prime}=\xi_{*}-\left[\left(\xi_{*}-\xi\right) \cdot \alpha\right] \alpha,
$$

where $X_{i}\left(=L x_{i}\right)$ (or $X(=L \boldsymbol{x})$ ) is the space rectangular coordinate system (or the position vector), $\boldsymbol{\alpha}$ is the unit vector, $\mathrm{d} \Omega(\boldsymbol{\alpha})$ is the solid angle element in the direction of $\boldsymbol{\alpha}$ and $\mathcal{B}$ is a non-negative function of $|\alpha \cdot V| /|V|$ and $|V|$ with $V=\xi_{*}-\xi$, whose functional form is determined by the intermolecular force. The domain of integration in (A2) is the whole space for $\boldsymbol{\xi}_{*}$ and all directions for $\boldsymbol{\alpha}$. Note that the dependency of $f$ on $X$ is not shown in (A2).

Let

$$
\mathcal{B}_{0}=\int \frac{1}{\left(2 \pi R T_{0}\right)^{3}} \exp \left(-\frac{|\boldsymbol{\xi}|^{2}+\left|\boldsymbol{\xi}_{*}\right|^{2}}{2 R T_{0}}\right) \mathcal{B} \mathrm{d} \Omega(\boldsymbol{\alpha}) \mathrm{d} \boldsymbol{\xi}_{*} \mathrm{~d} \boldsymbol{\xi}
$$

where the domain of integration is the whole space for $\boldsymbol{\xi}$ and $\xi_{*}$ and all directions for $\alpha$. Then, the dimensionless counterpart $\hat{\mathcal{B}}$ of $\mathcal{B}$ is defined by

$$
\mathcal{B}_{0}^{-1} \mathcal{B}=\hat{\mathcal{B}}\left(\frac{|\alpha \cdot \hat{V}|}{|\hat{V}|},|\hat{V}|\right), \quad \hat{V}=\frac{V}{\left(2 R T_{0}\right)^{1 / 2}}=\zeta_{*}-\zeta
$$

With this relation and the variables introduced in (2.1), the Boltzmann equation (A1) is transformed into the dimensionless form (2.3), where the operators $\mathscr{L}(\phi)$ and $\mathscr{J}(\phi, \phi)$ are given by

$$
\begin{gathered}
\mathscr{L}(\phi)=\int E_{*}\left(\phi\left(\zeta^{\prime}\right)+\phi\left(\zeta_{*}^{\prime}\right)-\phi(\zeta)-\phi\left(\zeta_{*}\right)\right) \hat{\mathcal{B}} \mathrm{d} \Omega(\boldsymbol{\alpha}) \mathrm{d} \zeta_{*}, \\
\mathscr{J}(\phi, \phi)=\int E_{*}\left(\phi\left(\zeta^{\prime}\right) \phi\left(\zeta_{*}^{\prime}\right)-\phi(\zeta) \phi\left(\zeta_{*}\right)\right) \hat{\mathcal{B}} \mathrm{d} \Omega(\boldsymbol{\alpha}) \mathrm{d} \zeta_{*},
\end{gathered}
$$

with

$$
\zeta^{\prime}=\zeta+\left[\left(\zeta_{*}-\zeta\right) \cdot \alpha\right] \alpha, \quad \zeta_{*}^{\prime}=\zeta_{*}-\left[\left(\zeta_{*}-\zeta\right) \cdot \alpha\right] \alpha,
$$

and $E_{*}=\pi^{-3 / 2} \exp \left(-\left|\zeta_{*}\right|^{2}\right)$. The domain of integration in (A6a) and (A6b) is the whole space for $\zeta_{*}$ and all directions for $\alpha$. Note that the dependency of $\phi$ on $\boldsymbol{x}$ is not shown. by

The symmetric bilinear operator associated with the quadratic form $\mathscr{J}(\phi, \phi)$ is given

$$
\begin{aligned}
\mathscr{J}(\psi, \phi)= & \frac{1}{2}(\mathscr{J}(\psi+\phi, \psi+\phi)-\mathscr{J}(\psi, \psi)-\mathscr{J}(\phi, \phi)) \\
= & \frac{1}{2} \int E_{*}\left(\psi\left(\zeta^{\prime}\right) \phi\left(\zeta_{*}^{\prime}\right)+\phi\left(\zeta^{\prime}\right) \psi\left(\zeta_{*}^{\prime}\right)-\psi(\zeta) \phi\left(\zeta_{*}\right)\right. \\
& \left.-\phi(\zeta) \psi\left(\zeta_{*}\right)\right) \hat{\mathcal{B}} \mathrm{d} \Omega(\alpha) \mathrm{d} \zeta_{*} .
\end{aligned}
$$

Note that $\mathscr{L}(\phi)=2 \mathscr{J}(1, \phi)$ holds.

The molecular mean free path in the equilibrium state at rest with density $\rho_{0}$ and temperature $T_{0}$ is given by $\ell_{0}=(2 / \sqrt{\pi})\left(2 R T_{0}\right)^{1 / 2} /\left[\left(\rho_{0} / m\right) \mathcal{B}_{0}\right]$ (e.g. Sone 2007). For a gas consisting of hard-sphere molecules with diameter $d_{m}$ (i.e. a hard-sphere gas), $\mathcal{B}$ is given by $\mathcal{B}=\left(d_{m}^{2} / 2\right)|\boldsymbol{\alpha} \cdot V|$. Therefore, we have

$$
\mathcal{B}_{0}=2 \sqrt{2 \pi} d_{m}^{2}\left(2 R T_{0}\right)^{1 / 2}, \quad \hat{\mathcal{B}}=\frac{|\boldsymbol{\alpha} \cdot \hat{V}|}{4 \sqrt{2 \pi}},
$$

for this model, and the mean free path is given by $\ell_{0}=1 /\left[\sqrt{2} \pi d_{m}^{2}\left(\rho_{0} / m\right)\right]$. 


\section{S. Taguchi and T. Tsuji}

The (time-independent) BGK model of the Boltzmann equation is obtained by replacing $J(f, f)$ in (A1) with

$$
\begin{gathered}
Q^{B G K}(f) \equiv A_{c} \rho\left(f_{e}-f\right), \\
f_{e}=\frac{\rho}{(2 \pi R T)^{3 / 2}} \exp \left(-\frac{\left(\xi_{i}-v_{i}\right)^{2}}{2 R T}\right), \\
\rho=\int f \mathrm{~d} \xi, \quad v_{i}=\frac{1}{\rho} \int \xi_{i} f \mathrm{~d} \xi, \quad T=\frac{1}{3 R \rho} \int\left(\xi_{i}-v_{i}\right)^{2} f \mathrm{~d} \xi,
\end{gathered}
$$

$(\mathrm{A} 12 a-c)$

where $A_{c}$ is a constant and $\rho, v_{i}$ and $T$ are the local density, the flow velocity and the temperature of the gas, respectively. The integrals with respect to $\xi$ in (A12) are carried out over the whole space. With the dimensionless quantities introduced in (2.1), the dimensionless form of the BGK equation is obtained as

$$
\begin{aligned}
\zeta_{i} \frac{\partial \phi}{\partial x_{i}} & =\frac{1}{k} \mathcal{Q}^{B G K}(\phi), \\
\mathcal{Q}^{B G K}(\phi) & =(1+\omega)\left(\phi_{e}-\phi\right),
\end{aligned}
$$

where $\phi_{e}$ is defined in (2.15). Linearizing $\mathcal{Q}^{B G K}(\phi)$ around the reference state, we obtain the well-known linearized BGK collision operator $\mathscr{L}^{B G K}(\phi)$ as shown in (2.12). On the other hand, we define $\mathscr{J}^{B G K}(\phi)$ as the remainder of $\mathcal{Q}^{B G K}(\phi)$ subtracted by $\mathscr{L}^{B G K}(\phi)$. We then obtain (2.13).

\section{Appendix B. Axial symmetry of the operators $\mathscr{L}$ and $\mathscr{J}$}

Let $a_{i}(i=1,2,3)$ be any fixed unit vector and let $\zeta_{\|}=\zeta_{i} a_{i}, \bar{\zeta}_{i}=\zeta_{j}\left(\delta_{i j}-a_{i} a_{j}\right)$ and $\zeta=$ $|\zeta|=\left(\zeta_{\|}^{2}+\bar{\zeta}_{i}^{2}\right)^{1 / 2}$. For any function $f$ of $\zeta_{\|}$and $\zeta, \mathscr{L}(f), \mathscr{L}\left(\bar{\zeta}_{i} f\right)$ and $\mathscr{L}\left(\bar{\zeta}_{i} \bar{\zeta}_{j} f\right), i, j=$ $1,2,3$, are expressed in the following forms (Sone 2002, 2007):

$$
\begin{gathered}
\mathscr{L}(f)=\mathscr{L}_{0}(f), \\
\mathscr{L}\left(\bar{\zeta}_{i} f\right)=\bar{\zeta}_{i} \mathscr{L}_{1}(f), \\
\mathscr{L}\left(\bar{\zeta}_{i} \bar{\zeta}_{j} f\right)=\bar{\zeta}_{i} \bar{\zeta}_{j} \mathscr{L}_{2}(f)+\left(\delta_{i j}-a_{i} a_{j}\right) \mathscr{L}_{3}(f),
\end{gathered}
$$

where $\mathscr{L}_{l}(f), l=0,1,2,3$, are functions of $\zeta_{\|}$and $\zeta$. Also, for any functions $f$ and $g$ of $\zeta_{\|}$and $\zeta, \mathscr{J}(f, g), \mathscr{J}\left(f, \bar{\zeta}_{i} g\right), \frac{1}{2}\left(\mathscr{J}\left(\bar{\zeta}_{i} f, \bar{\zeta}_{j} g\right)+\mathscr{J}\left(\bar{\zeta}_{j} f, \bar{\zeta}_{i} g\right)\right)$ and $\frac{1}{2}\left(\mathscr{J}\left(\bar{\zeta}_{i} f, \bar{\zeta}_{j} g\right)-\right.$ $\left.\mathscr{J}\left(\bar{\zeta}_{j} f, \bar{\zeta}_{i} g\right)\right), i, j=1,2,3$, are expressed in the following forms (Sone 2002, 2007):

$$
\begin{gathered}
\mathscr{J}(f, g)=\mathscr{J}_{0}(f, g), \\
\mathscr{J}\left(f, \bar{\zeta}_{i} g\right)=\mathscr{J}\left(\bar{\zeta}_{i} g, f\right)=\bar{\zeta}_{i} \mathscr{J}_{1}(f, g), \\
\frac{1}{2}\left(\mathscr{J}\left(\bar{\zeta}_{i} f, \bar{\zeta}_{j} g\right)+\mathscr{J}\left(\bar{\zeta}_{j} f, \bar{\zeta}_{i} g\right)\right)=\bar{\zeta}_{i} \bar{\zeta}_{j} \mathscr{J}_{2}(f, g)+\left(\delta_{i j}-a_{i} a_{j}\right) \mathscr{J}_{3}(f, g), \\
\frac{1}{2}\left(\mathscr{J}\left(\bar{\zeta}_{i} f, \bar{\zeta}_{j} g\right)-\mathscr{J}\left(\bar{\zeta}_{j} f, \bar{\zeta}_{i} g\right)\right)=\epsilon_{i j m} a_{m} \zeta_{\|} \mathscr{J}_{4}(f, g),
\end{gathered}
$$

where $\mathscr{J}_{l}(f, g), l=0,1,2,3,4$, are functions of $\zeta_{\|}$and $\zeta$. Note that $\mathscr{J}_{0}(f, g), \mathscr{J}_{2}(f, g)$ and $\mathscr{J}_{3}(f, g)$ are symmetric and $\mathscr{J}_{4}(f, g)$ is antisymmetric with respect to interchange 


\section{Inversion of the transverse force on a spinning sphere}

of $f$ and $g$, i.e.

$$
\mathscr{J}_{l}(f, g)=\mathscr{J}_{l}(g, f), \quad l=0,2,3, \quad \mathscr{J}_{4}(f, g)=-\mathscr{J}_{4}(g, f) \quad\left(\mathscr{J}_{4}(f, f)=0\right) .
$$

$(\mathrm{B} 3 a, b)$

From $(\mathrm{B} 1 c)$, we have

$$
\mathscr{L}\left(\bar{\zeta}_{k}^{2} f\right)=\bar{\zeta}_{k}^{2} \mathscr{L}_{2}(f)+2 \mathscr{L}_{3}(f) .
$$

Multiplying this by $\frac{1}{2}\left(\delta_{i j}-a_{i} a_{j}\right)$ and subtracting the result from $(\mathrm{B} 1 c)$, we obtain

$$
\mathscr{L}\left(\left(\bar{\zeta}_{i} \bar{\zeta}_{j}-\frac{\bar{\zeta}_{k}^{2}}{2}\left(\delta_{i j}-a_{i} a_{j}\right)\right) f\right)=\left(\bar{\zeta}_{i} \bar{\zeta}_{j}-\frac{\bar{\zeta}_{k}^{2}}{2}\left(\delta_{i j}-a_{i} a_{j}\right)\right) \mathscr{L}_{2}(f) .
$$

Next, adding both sides of (B2c) and (B2d), we obtain

$$
\mathscr{J}\left(\bar{\zeta}_{i} f, \bar{\zeta}_{j} g\right)=\bar{\zeta}_{i} \bar{\zeta}_{j} \mathscr{J}_{2}(f, g)+\left(\delta_{i j}-a_{i} a_{j}\right) \mathscr{J}_{3}(f, g)+\epsilon_{i j m} a_{m} \zeta_{\|} \mathscr{J}_{4}(f, g) \text {. }
$$

In our spherical coordinate system, it follows from (B1b) and (B5) that

$$
\left.\begin{array}{c}
\mathscr{L}\left(\zeta_{\theta} F\right)=\zeta_{\theta} \mathscr{L}_{1}(F), \quad \mathscr{L}\left(\zeta_{\varphi} F\right)=\zeta_{\varphi} \mathscr{L}_{1}(F), \\
\mathscr{L}\left(\frac{\zeta_{\theta}^{2}-\zeta_{\varphi}^{2}}{2} F\right)=\frac{\zeta_{\theta}^{2}-\zeta_{\varphi}^{2}}{2} \mathscr{L}_{2}(F), \quad \mathscr{L}\left(\zeta_{\theta} \zeta_{\varphi} F\right)=\zeta_{\theta} \zeta_{\varphi} \mathscr{L}_{2}(F),
\end{array}\right\}
$$

where $F$ is any function of $\zeta_{r}$ and $\zeta$. From (B2b) and (B6), it follows that

$$
\left.\begin{array}{c}
\mathscr{J}\left(F, \zeta_{\theta} G\right)=\zeta_{\theta} \mathscr{J}_{1}(F, G), \quad \mathscr{J}\left(F, \zeta_{\varphi} G\right)=\zeta_{\varphi} \mathscr{J}_{1}(F, G), \\
\mathscr{J}\left(\zeta_{\theta} F, \zeta_{\theta} G\right)=\zeta_{\theta}^{2} \mathscr{J}_{2}(F, G)+\mathscr{J}_{3}(F, G), \\
\mathscr{J}\left(\zeta_{\varphi} F, \zeta_{\varphi} G\right)=\zeta_{\varphi}^{2} \mathscr{J}_{2}(F, G)+\mathscr{J}_{3}(F, G), \\
\mathscr{J}\left(\zeta_{\theta} F, \zeta_{\varphi} G\right)=\zeta_{\theta} \zeta_{\varphi} \mathscr{J}_{2}(F, G)+\zeta_{r} \mathscr{J}_{4}(F, G),
\end{array}\right\}
$$

where $F$ and $G$ are any functions of $\zeta_{r}$ and $\zeta$.

For the BGK collision model, $\mathscr{L}$ and $\mathscr{J}$ in the above formulas should be replaced by $\mathscr{L}^{B G K}$ (see (2.12)) and $\mathscr{J}^{B G K}$ (see (4.3)), respectively. If we denote the corresponding counterparts of $\mathscr{L}_{l}(l=0,1,2,3)$ and $\mathscr{J}_{l}(l=0,1,2,3,4)$ by $\mathscr{L}_{l}^{B G K}$ and $\mathscr{J}_{l}^{B G K}$, the formulas (B7) and (B8) hold with

$$
\begin{gathered}
\mathscr{L}_{0}^{B G K}(F)=\tilde{\omega}[F]+2 \zeta_{r} \tilde{u}_{r}[F]+\left(\zeta^{2}-\frac{3}{2}\right) \tilde{\tau}[F]-F \\
\mathscr{L}_{1}^{B G K}(F)=2 \tilde{u}_{t}[F]-F \\
\mathscr{L}_{2}^{B G K}(F)=-F \\
\mathscr{L}_{3}^{B G K}(F)=\tilde{u}_{t}[F]+\zeta_{r} \tilde{u}_{r}\left[\left(\zeta^{2}-\zeta_{r}^{2}\right) F\right]+\frac{1}{2}\left(\zeta^{2}-\frac{3}{2}\right) \tilde{\tau}\left[\left(\zeta^{2}-\zeta_{r}^{2}\right) F\right] \\
\mathscr{J}_{0}^{B G K}(F, G)= \\
+\frac{2}{3}\left(3 \zeta_{r}^{2}-\zeta^{2}\right) \tilde{u}_{r}[F] \tilde{u}_{r}[G]+\zeta_{r}\left(\zeta^{2}-\frac{5}{2}\right)\left(\tilde{u}_{r}[F] \tilde{\tau}[G]+\tilde{u}_{r}[G] \tilde{\tau}[F]\right) \\
+\frac{1}{2}\left(\tilde{\omega}[F] \mathscr{L}_{0}^{B G K}(G)+\tilde{\omega}[G] \mathscr{L}_{0}^{B G K}(F)\right) \\
\mathscr{\mathscr { J }}_{1}^{B G K}(F, G)= \\
2 \tilde{u}_{t}[G]\left[\zeta_{r} \tilde{u}_{r}[F]+\frac{1}{2}\left(\zeta^{2}-\frac{5}{2}\right) \tilde{\tau}[F]\right]+\frac{1}{2} \tilde{\omega}[F] \mathscr{L}_{1}^{B G K}(G)
\end{gathered}
$$




$$
\begin{gathered}
\text { S. Taguchi and T. Tsuji } \\
\mathscr{J}_{2}^{B G K}(F, G)=2 \tilde{u}_{t}[F] \tilde{u}_{t}[G], \\
\mathscr{J}_{3}^{B G K}(F, G)=-\frac{2}{3} \zeta^{2} \tilde{u}_{t}[F] \tilde{u}_{t}[G], \\
\mathscr{J}_{4}^{B G K}(F, G)=0,
\end{gathered}
$$

where $\tilde{\omega}$ and $\tilde{\tau}$ (or $\tilde{u}_{r}$ and $\tilde{u}_{t}$ ) are given by (3.19a) (or (3.21a)).

\section{Appendix C. Functions related to the linearized collision operator $\mathscr{L}$}

Let us introduce functions $A(\zeta), B(\zeta), D_{1}(\zeta), D_{2}(\zeta)$ and $F(\zeta)$ as the solutions to the following equations:

$$
\begin{gathered}
\mathscr{L}\left(\zeta_{i} A\right)=-\zeta_{i}\left(\zeta^{2}-\frac{5}{2}\right) \\
\mathscr{L}\left(\left(\zeta_{i} \zeta_{j}-\frac{\zeta^{2}}{3} \delta_{i j}\right) B\right)=-2\left(\zeta_{i} \zeta_{j}-\frac{\zeta^{2}}{3} \delta_{i j}\right), \\
\mathscr{L}\left(\left(\zeta_{i} \delta_{j k}+\zeta_{j} \delta_{k i}+\zeta_{k} \delta_{i j}\right) D_{1}+\zeta_{i} \zeta_{j} \zeta_{k} D_{2}\right) \\
=\gamma_{1}\left(\zeta_{i} \delta_{j k}+\zeta_{j} \delta_{k i}+\zeta_{k} \delta_{i j}\right)-\zeta_{i} \zeta_{j} \zeta_{k} B \\
\mathscr{L}\left(\left(\zeta_{i} \zeta_{j}-\frac{\zeta^{2}}{3} \delta_{i j}\right) F\right)=\left(\zeta_{i} \zeta_{j}-\frac{\zeta^{2}}{3} \delta_{i j}\right) A,
\end{gathered}
$$

where $\gamma_{1}$ is defined in $(3.39 a)$, and $A(\zeta), D_{1}(\zeta)$ and $D_{2}(\zeta)$ are subject to the following subsidiary conditions:

$$
\left\langle\zeta^{2} A\right\rangle=\left\langle 5 \zeta^{2} D_{1}+\zeta^{4} D_{2}\right\rangle=0 .
$$

With $A(\zeta)$ and $B(\zeta)$ introduced above, functions $C(\zeta)$ and $G(\zeta)$ are defined by the relations

$$
\begin{aligned}
& 2 \mathscr{J}\left(\zeta^{2}-\frac{3}{2},\right.\left.\left(\zeta_{i} \zeta_{j}-\frac{\zeta^{2}}{3} \delta_{i j}\right) B\right)=\left(\zeta_{i} \zeta_{j}-\frac{\zeta^{2}}{3} \delta_{i j}\right) C, \\
& 2 \mathscr{J}\left(\zeta^{2}-\frac{3}{2}, \zeta_{i} A\right)=\zeta_{i} G .
\end{aligned}
$$

For the BGK model, the functions $A, \ldots, F$ are explicitly given by

$$
\left.\begin{array}{c}
A(\zeta)=\zeta^{2}-\frac{5}{2}, \quad B(\zeta)=2, \quad D_{1}(\zeta)=-1, \\
D_{2}(\zeta)=2, \quad F(\zeta)=-\zeta^{2}+\frac{5}{2} .
\end{array}\right\}
$$

\section{Appendix D. Proofs of Propositions 3.1 and 3.2}

In this appendix, we present the proofs of Propositions 3.1 and 3.2.

\section{Proof of Proposition 3.1}

We recall the relations (3.10) and (3.16) and go back to the original problem (3.6)-(3.9) for $\phi_{F 1}$. Consider a large sphere (say, $\left.S_{0}\right)$ with radius $r_{0}(>1)$ centred at the origin, enclosing the original sphere inside. For a sufficiently large $r_{0}$, the length scale of variation of $\phi_{F 1}$ is of the order of $r_{0}$ near the surface of $S_{0}$. Since $r_{0}$ can be taken arbitrarily large, we can assume that the local Knudsen number, $k / r_{0}$, is small near the surface of $S_{0}$. Consequently, the behaviour of the gas is described by the Grad-Hilbert expansion 


\section{Inversion of the transverse force on a spinning sphere}

(e.g. Sone 2007, Chap. 3) there. As a result, the macroscopic variables $\omega_{F 1}, u_{i F 1}, \tau_{F 1}, P_{F 1}$ are described by the following partial differential equations (i.e. Stokes-type equations):

$$
\begin{gathered}
\frac{\partial u_{i F 1}}{\partial x_{i}}=0, \\
\gamma_{1} k \Delta u_{i F 1}-\frac{\partial P_{F 1}}{\partial x_{i}}=0, \\
\Delta \tau_{F 1}=0, \\
\omega_{F 1}=P_{F 1}-\tau_{F 1},
\end{gathered}
$$

where $\Delta=\partial^{2} / \partial x_{j}^{2}$ is the Laplacian operator and $\gamma_{1}$ is the constant defined in $(3.39 a)$. Moreover, with $P_{F 1}, u_{i F 1}$ and $\tau_{F 1}$ that satisfy (D1), the velocity distribution is expressed in the form

$$
\begin{aligned}
\phi_{F 1}= & P_{F 1}+2 \zeta_{i} u_{i F 1}+\left(\zeta^{2}-\frac{5}{2}\right) \tau_{F 1} \\
& -\frac{k}{2} \zeta_{i} \zeta_{j} B(\zeta)\left(\frac{\partial u_{i F 1}}{\partial x_{j}}+\frac{\partial u_{j F 1}}{\partial x_{i}}\right)-k \zeta_{i} A(\zeta) \frac{\partial \tau_{F 1}}{\partial x_{i}} \\
& +\frac{k^{2}}{2}\left[\left(\zeta_{i} \delta_{j l}+\zeta_{j} \delta_{l i}+\zeta_{l} \delta_{i j}\right) D_{1}(\zeta)+\zeta_{i} \zeta_{j} \zeta_{l} D_{2}(\zeta)\right] \frac{\partial}{\partial x_{i}}\left(\frac{\partial u_{l F 1}}{\partial x_{j}}+\frac{\partial u_{j F 1}}{\partial x_{l}}\right) \\
& -k^{2} \zeta_{i} \zeta_{j} F(\zeta) \frac{\partial^{2} \tau_{F 1}}{\partial x_{i} \partial x_{j}}+\cdots,
\end{aligned}
$$

where the functions $A(\zeta), B(\zeta), \ldots, F(\zeta)$ are defined in Appendix C. Because of the condition (3.9), $P_{F 1}, u_{i F 1}$ and $\tau_{F 1}$ contained in (D2) are required to satisfy the condition

$$
P_{F 1} \rightarrow 0, \quad\left(u_{i F 1}\right)_{i=1,2,3} \rightarrow(U, V, 0), \quad \tau_{F 1} \rightarrow 0, \quad \text { as }|x| \rightarrow \infty . \quad(\mathrm{D} 3 a-c)
$$

This serves as boundary conditions for (D1) at infinity.

Now we introduce (3.10) together with the similarity forms (3.16). Consequently, $P_{F 1}$, $u_{i F 1}$ and $\tau_{F 1}$ take the forms given by $(3.22 b)-(3.22 f)$. Substituting them into (D1), we obtain a set of ordinary differential equations for $\tilde{u}_{r, \mathrm{U} a}^{(1)}(r), \tilde{u}_{t, \mathrm{U} b}^{(1)}(r), \tilde{P}_{\mathrm{U} a}^{(1)}(r), \tilde{\tau}_{\mathrm{U} a}^{(1)}(r)$ and $\tilde{u}_{t, \mathrm{~S}}^{(1)}(r)$ (we omit the resulting equations for conciseness). The most general solution satisfying the condition at infinity (D 3 ) is given by

$$
\left.\begin{array}{c}
\tilde{u}_{r, \mathrm{U} a}^{(1)}(r)=1+\frac{c_{1}}{r}+\frac{c_{2}}{r^{3}}, \quad \tilde{u}_{t, \mathrm{U} b}^{(1)}(r)=-1-\frac{c_{1}}{2 r}+\frac{c_{2}}{2 r^{3}}, \\
\tilde{P}_{\mathrm{U} a}^{(1)}(r)=\gamma_{1} k \frac{c_{1}}{r^{2}}, \quad \tilde{\tau}_{\mathrm{U} a}^{(1)}(r)=\frac{c_{3}}{r^{2}}, \quad \tilde{u}_{t, \mathrm{~S}}^{(1)}(r)=\frac{c_{4}}{r^{2}},
\end{array}\right\}
$$

where $c_{i}, i=1, \ldots, 4$, are undetermined constants. Meanwhile, if we use (3.22b)-(3.22f) together with (3.10) and (3.16) in (D2), we obtain the expressions of $\varphi_{\mathrm{U} a}^{(1)}, \varphi_{\mathrm{U} b}^{(1)}$ and $\varphi_{\mathrm{S}}^{(1)}$ in terms of $\tilde{u}_{r, \mathrm{U} a}^{(1)}, \tilde{u}_{t, \mathrm{U} b}^{(1)}, \tilde{P}_{\mathrm{U} a}^{(1)}, \tilde{\tau}_{\mathrm{U} a}^{(1)}$ and $\tilde{u}_{t, \mathrm{~S}}^{(1)}$. Substituting (D4) into $\varphi_{\mathrm{U} a}^{(1)}, \varphi_{\mathrm{U} b}^{(1)}$ and $\varphi_{\mathrm{S}}^{(1)}$ thus obtained, we arrive at the expressions (3.47)-(3.49). This concludes the proof.

\section{Proof of Proposition 3.2}

Multiplying (3.6) by $\zeta_{i}$ and integrating over $\zeta$, we obtain $\partial P_{i j F 1} / \partial x_{j}=0$ everywhere in the gas region. We then multiply 1 or $\epsilon_{l k i} x_{k}$ to this and integrate the result over 


\section{S. Taguchi and T. Tsuji}

the domain enclosed by the original sphere (radius 1) and the sphere $S_{0}$ (radius $r_{0}$ ) considered in Proposition 3.1 to obtain $\mathcal{F}_{i}^{(1)}=-\int_{|x|=r_{0}} P_{i j F 1} n_{j} \mathrm{~d} S$ and $\mathcal{M}_{i}^{(1)}=$ $-\int_{|x|=r_{0}} \epsilon_{i j m} x_{j} P_{m l F 1} n_{l} \mathrm{~d} S$, where $n_{i}$ is the outward unit normal vector on $S_{0}$ and $\mathcal{F}_{i}^{(1)}$ and $\mathcal{M}_{i}^{(1)}$ are defined in $(3.26 a, b)$. Using $(3.22 g)-(3.22 i)$, this in turn implies that

$$
\begin{gathered}
h_{D}=-\frac{4}{3} \pi r_{0}^{2}\left(\tilde{P}_{r r, \mathrm{U} a}^{(1)}\left(r_{0}\right)-2 \tilde{P}_{r t, \mathrm{U} b}^{(1)}\left(r_{0}\right)\right), \\
h_{M}=-\frac{8}{3} \pi r_{0}^{3} \tilde{P}_{r t, \mathrm{~S}}^{(1)}\left(r_{0}\right) .
\end{gathered}
$$

Now, direct computations using (3.47)-(3.49) show that $\tilde{P}_{r r, \mathrm{U} a}^{(1)}, \tilde{P}_{r t, \mathrm{U} b}^{(1)}$ and $\tilde{P}_{r t, \mathrm{~S}}^{(1)}$ (see (3.23)) have the following asymptotic properties for large $r \gg 1$ :

$$
\tilde{P}_{r r, \mathrm{U} a}^{(1)}=\frac{3 \gamma_{1} k c_{1}}{r^{2}}+O\left(r^{-4}\right), \quad \tilde{P}_{r t, \mathrm{U} b}^{(1)}=O\left(r^{-4}\right), \quad \tilde{P}_{r t, \mathrm{~S}}^{(1)}=\frac{3 \gamma_{1} k c_{4}}{r^{3}}, \quad r \gg 1 . \quad(\mathrm{D} 7 a-c)
$$

Substituting (D 7) with $r=r_{0}$ into (D5) and (D6) and letting $r_{0} \rightarrow \infty$, we obtain $(3.50 a, b)$.

\section{Appendix E. Boundary-value problems for the second-order inner problem}

In this appendix, we show boundary-value problems for $\left(\varphi_{\mathrm{UU} \alpha}^{(2)}, \varphi_{\mathrm{SS} \alpha}^{(2)}, \varphi_{\mathrm{US} \alpha}^{(2) \sharp}, \varphi_{\mathrm{US} \alpha}^{(2) b}\right), \alpha=$ $a, b, c, d$, appearing in the similarity solutions (3.79)-(3.83). They are summarized as follows.

(a) Problem UU

$$
\begin{gathered}
\zeta_{r} \frac{\partial \varphi_{\mathrm{UU} a}^{(2)}}{\partial r}+\frac{\zeta^{2}-\zeta_{r}^{2}}{r} \frac{\partial \varphi_{\mathrm{UU} a}^{(2)}}{\partial \zeta_{r}}+\frac{3}{2} \frac{\zeta^{2}-\zeta_{r}^{2}}{r} \varphi_{\mathrm{UU} b}^{(2)}=\frac{1}{k} \mathscr{L}_{0}\left(\varphi_{\mathrm{UU} a}^{(2)}\right)+\frac{1}{k} I_{\mathrm{UU} a}^{(2)}, \\
\zeta_{r} \frac{\partial \varphi_{\mathrm{UU} b}^{(2)}}{\partial r}+\frac{\zeta^{2}-\zeta_{r}^{2}}{r} \frac{\partial \varphi_{\mathrm{UU} b}^{(2)}}{\partial \zeta_{r}}-\frac{\zeta_{r}}{r} \varphi_{\mathrm{UU} b}^{(2)}-\frac{2}{r} \varphi_{\mathrm{UU} a}^{(2)}+\frac{\zeta^{2}-\zeta_{r}^{2}}{r} \varphi_{\mathrm{UU} c}^{(2)} \\
=\frac{1}{k} \mathscr{L}_{1}\left(\varphi_{\mathrm{UU} b}^{(2)}\right)+\frac{1}{k} I_{\mathrm{UU} b}^{(2)}, \\
\zeta_{r} \frac{\partial \varphi_{\mathrm{UU} c}^{(2)}}{\partial r}+\frac{\zeta^{2}-\zeta_{r}^{2}}{r} \frac{\partial \varphi_{\mathrm{UU} c}^{(2)}}{\partial \zeta_{r}}-2 \frac{\zeta_{r}}{r} \varphi_{\mathrm{UU} c}^{(2)}-\frac{\varphi_{\mathrm{UU} b}^{(2)}}{r}=\frac{1}{k} \mathscr{L}_{2}\left(\varphi_{\mathrm{UU} c}^{(2)}\right)+\frac{1}{k} I_{\mathrm{UU} c}^{(2)} \\
\zeta_{r} \frac{\partial \varphi_{\mathrm{UU} d}^{(2)}}{\partial r}+\frac{\zeta^{2}-\zeta_{r}^{2}}{r} \frac{\partial \varphi_{\mathrm{UU} d}^{(2)}}{\partial \zeta_{r}}-\frac{\zeta^{2}-\zeta_{r}^{2}}{2 r} \varphi_{\mathrm{UU} b}^{(2)}=\frac{1}{k} \mathscr{L}_{0}\left(\varphi_{\mathrm{UU} d}^{(2)}\right)+\frac{1}{k} I_{\mathrm{UU} d}^{(2)}, \\
\text { b.c. }\left\{\begin{array}{l}
\varphi_{\mathrm{UU} a}^{(2)}=\mathscr{K}\left(\varphi_{\mathrm{UU} a}^{(2)}\right), \\
\varphi_{\mathrm{UU} b}^{(2)}=0, \\
\varphi_{\mathrm{UU} c}^{(2)}=0, \\
\varphi_{\mathrm{UU} d}^{(2)}=\mathscr{K}\left(\varphi_{\mathrm{UU} d}^{(2)}\right),
\end{array} \quad r=1,\right. \\
\zeta_{r}>0,
\end{gathered}
$$




$$
\text { b.c. }\left\{\begin{array}{l}
\varphi_{\mathrm{UU} a}^{(2)} \rightarrow \frac{3}{2} \frac{c_{1}}{\gamma_{1} k} \zeta_{r}+3 \zeta_{r}^{2}-\zeta^{2}, \\
\varphi_{\mathrm{UU} b}^{(2)} \rightarrow-\frac{c_{1}}{\gamma_{1} k}-4 \zeta_{r}, \\
\varphi_{\mathrm{UU} c}^{(2)} \rightarrow 2, \\
\varphi_{\mathrm{UU} d}^{(2)} \rightarrow-\frac{c_{1}}{2 \gamma_{1} k} \zeta_{r}+\zeta^{2}-\zeta_{r}^{2}-1,
\end{array} \quad \text { as } r \rightarrow \infty .\right.
$$

(b) Problem SS

$$
\begin{aligned}
& \zeta_{r} \frac{\partial \varphi_{\mathrm{SS} a}^{(2)}}{\partial r}+\frac{\zeta^{2}-\zeta_{r}^{2}}{r} \frac{\partial \varphi_{\mathrm{SS} a}^{(2)}}{\partial \zeta_{r}}+\frac{3}{2} \frac{\zeta^{2}-\zeta_{r}^{2}}{r} \varphi_{\mathrm{SS} b}^{(2)}=\frac{1}{k} \mathscr{L}_{0}\left(\varphi_{\mathrm{SS} a}^{(2)}\right)+\frac{1}{k} I_{\mathrm{SS} a}^{(2)}, \\
& \zeta_{r} \frac{\partial \varphi_{\mathrm{SS} b}^{(2)}}{\partial r}+\frac{\zeta^{2}-\zeta_{r}^{2}}{r} \frac{\partial \varphi_{\mathrm{SS} b}^{(2)}}{\partial \zeta_{r}}-\frac{\zeta_{r}}{r} \varphi_{\mathrm{SS} b}^{(2)}-\frac{2}{r} \varphi_{\mathrm{SS} a}^{(2)}+\frac{\zeta^{2}-\zeta_{r}^{2}}{r} \varphi_{\mathrm{SS} c}^{(2)} \\
& =\frac{1}{k} \mathscr{L}_{1}\left(\varphi_{\mathrm{SS} b}^{(2)}\right)+\frac{1}{k} I_{\mathrm{SS} b}^{(2)} \text {, } \\
& \zeta_{r} \frac{\partial \varphi_{\mathrm{SS} c}^{(2)}}{\partial r}+\frac{\zeta^{2}-\zeta_{r}^{2}}{r} \frac{\partial \varphi_{\mathrm{SS} c}^{(2)}}{\partial \zeta_{r}}-2 \frac{\zeta_{r}}{r} \varphi_{\mathrm{SS} c}^{(2)}-\frac{\varphi_{\mathrm{SS} b}^{(2)}}{r}=\frac{1}{k} \mathscr{L}_{2}\left(\varphi_{\mathrm{SS} c}^{(2)}\right)+\frac{1}{k} I_{\mathrm{SS} c}^{(2)} \\
& \zeta_{r} \frac{\partial \varphi_{\mathrm{SS} d}^{(2)}}{\partial r}+\frac{\zeta^{2}-\zeta_{r}^{2}}{r} \frac{\partial \varphi_{\mathrm{SS} d}^{(2)}}{\partial \zeta_{r}}-\frac{\zeta^{2}-\zeta_{r}^{2}}{2 r} \varphi_{\mathrm{SS} b}^{(2)}=\frac{1}{k} \mathscr{L}_{0}\left(\varphi_{\mathrm{SS} d}^{(2)}\right)+\frac{1}{k} I_{\mathrm{SS} d}^{(2)}, \\
& \text { b.c. }\left\{\begin{array}{l}
\varphi_{\mathrm{SS} a}^{(2)}=\mathscr{K}\left(\varphi_{\mathrm{SS} a}^{(2)}\right)-\left(\zeta^{2}-\zeta_{r}^{2}-1\right), \\
\varphi_{\mathrm{SS} b}^{(2)}=0, \\
\varphi_{\mathrm{SS} c}^{(2)}=-2, \\
\varphi_{\mathrm{SS} d}^{(2)}=\mathscr{K}\left(\varphi_{\mathrm{SS} d}^{(2)}\right)+\zeta^{2}-\zeta_{r}^{2}-1,
\end{array}\right. \\
& \text { b.c. } \quad \varphi_{\mathrm{SS} \alpha}^{(2)} \rightarrow 0 \quad \text { as } r \rightarrow \infty \quad(\alpha=a, b, c, d) .
\end{aligned}
$$

(c) Problem US

$$
\begin{aligned}
& \zeta_{r} \frac{\partial \varphi_{\mathrm{US} a}^{(2) \sharp}}{\partial r}+\frac{\zeta^{2}-\zeta_{r}^{2}}{r} \frac{\partial \varphi_{\mathrm{US} a}^{(2) \sharp}}{\partial \zeta_{r}}-\frac{\zeta_{r}}{r} \varphi_{\mathrm{US} a}^{(2) \sharp}+\frac{\zeta^{2}-\zeta_{r}^{2}}{2 r} \varphi_{\mathrm{US} c}^{(2) \sharp}+\frac{\varphi_{\mathrm{US} d}^{(2) \sharp}}{r} \\
& =\frac{1}{k} \mathscr{L}_{1}\left(\varphi_{\mathrm{US} a}^{(2) \sharp}\right)+\frac{1}{k} I_{\mathrm{US} a}^{(2) \sharp}, \\
& \zeta_{r} \frac{\partial \varphi_{\mathrm{US} b}^{(2) \sharp}}{\partial r}+\frac{\zeta^{2}-\zeta_{r}^{2}}{r} \frac{\partial \varphi_{\mathrm{US} b}^{(2) \sharp}}{\partial \zeta_{r}}-\frac{\zeta_{r}}{r} \varphi_{\mathrm{US} b}^{(2) \sharp}+\frac{\zeta^{2}-\zeta_{r}^{2}}{2 r} \varphi_{\mathrm{US} c}^{(2) \sharp}-\frac{\varphi_{\mathrm{US} d}^{(2) \sharp}}{r} \\
& =\frac{1}{k} \mathscr{L}_{1}\left(\varphi_{\mathrm{US} b}^{(2) \sharp}\right)+\frac{1}{k} I_{\mathrm{US} b}^{(2) \sharp},
\end{aligned}
$$




\section{S. Taguchi and T. Tsuji}

$$
\begin{gathered}
\zeta_{r} \frac{\partial \varphi_{\mathrm{US} c}^{(2) \sharp}}{\partial r}+\frac{\zeta^{2}-\zeta_{r}^{2}}{r} \frac{\partial \varphi_{\mathrm{US} c}^{(2) \sharp}}{\partial \zeta_{r}}-2 \frac{\zeta_{r}}{r} \varphi_{\mathrm{US} c}^{(2) \sharp}-\frac{\varphi_{\mathrm{US} a}^{(2)}}{r}-\frac{\varphi_{\mathrm{US} b}^{(2) \sharp}}{r}=\frac{1}{k} \mathscr{L}_{2}\left(\varphi_{\mathrm{US} c}^{(2) \sharp}\right)+\frac{1}{k} I_{\mathrm{UU} c}^{(2) \sharp}, \\
\zeta_{r} \frac{\partial \varphi_{\mathrm{US} d}^{(2) \sharp}}{\partial r}+\frac{\zeta^{2}-\zeta_{r}^{2}}{r} \frac{\partial \varphi_{\mathrm{US} d}^{(2) \sharp}}{\partial \zeta_{r}}-\frac{\zeta^{2}-\zeta_{r}^{2}}{2 r} \varphi_{\mathrm{US} a}^{(2) \sharp}+\frac{\zeta^{2}-\zeta_{r}^{2}}{2 r} \varphi_{\mathrm{US} b}^{(2) \sharp} \\
=\frac{1}{k} \mathscr{L}_{0}\left(\varphi_{\mathrm{US} d}^{(2) \sharp}\right)+\frac{1}{k} I_{\mathrm{US} d}^{(2) \sharp},
\end{gathered}
$$

b.c. $\left\{\begin{array}{l}\varphi_{\mathrm{US} a}^{(2) \sharp}=2 \mathscr{K}\left(\varphi_{\mathrm{U} a}^{(1)}\right), \\ \varphi_{\mathrm{US} b}^{(2) \sharp}=0, \\ \varphi_{\mathrm{US} c}^{(2) \sharp}=0, \\ \varphi_{\mathrm{US} d}^{(2) \sharp}=\mathscr{K}\left(\varphi_{\mathrm{US} d}^{(2) \sharp}\right),\end{array} \quad \zeta_{r}>0, \quad r=1\right.$,

b.c. $\quad \varphi_{\mathrm{US} \alpha}^{(2) \sharp} \rightarrow 0 \quad$ as $r \rightarrow \infty \quad(\alpha=a, b, c, d)$,

$$
\begin{aligned}
\zeta_{r} \frac{\partial \varphi_{\mathrm{US} a}^{(2) b}}{\partial r} & +\frac{\zeta^{2}-\zeta_{r}^{2}}{r} \frac{\partial \varphi_{\mathrm{US} a}^{(2) b}}{\partial \zeta_{r}}-3 \frac{\zeta^{2}-\zeta_{r}^{2}}{r} \varphi_{\mathrm{US} b}^{(2) b}=\frac{1}{k} \mathscr{L}_{0}\left(\varphi_{\mathrm{US} a}^{(2) b}\right)+\frac{1}{k} I_{\mathrm{US} a}^{(2) b}, \\
& \zeta_{r} \frac{\partial \varphi_{\mathrm{US} b}^{(2) b}}{\partial r}+\frac{\zeta^{2}-\zeta_{r}^{2}}{r} \frac{\partial \varphi_{\mathrm{US} b}^{(2) b}}{\partial \zeta_{r}}-\frac{\zeta_{r}}{r} \varphi_{\mathrm{US} b}^{(2) b}+\frac{\varphi_{\mathrm{US} a}^{(2) b}}{r}+\frac{\zeta^{2}-\zeta_{r}^{2}}{2 r} \varphi_{\mathrm{US} c}^{(2) b} \\
& =\frac{1}{k} \mathscr{L}_{1}\left(\varphi_{\mathrm{US} b}^{(2) b}\right)+\frac{1}{k} I_{\mathrm{US} b}^{(2) b},
\end{aligned}
$$

$$
\zeta_{r} \frac{\partial \varphi_{\mathrm{US} c}^{(2) b}}{\partial r}+\frac{\zeta^{2}-\zeta_{r}^{2}}{r} \frac{\partial \varphi_{\mathrm{US} c}^{(2) b}}{\partial \zeta_{r}}-2 \frac{\zeta_{r}}{r} \varphi_{\mathrm{US} c}^{(2) b}-2 \frac{\varphi_{\mathrm{US} b}^{(2) b}}{r}=\frac{1}{k} \mathscr{L}_{2}\left(\varphi_{\mathrm{US} c}^{(2) b}\right)+\frac{1}{k} I_{\mathrm{US} c}^{(2) b},
$$

$$
\zeta_{r} \frac{\partial \varphi_{\mathrm{US} d}^{(2) b}}{\partial r}+\frac{\zeta^{2}-\zeta_{r}^{2}}{r} \frac{\partial \varphi_{\mathrm{US} d}^{(2) b}}{\partial \zeta_{r}}+2 \frac{\zeta^{2}-\zeta_{r}^{2}}{r} \varphi_{\mathrm{US} b}^{(2) b}=\frac{1}{k} \mathscr{L}_{0}\left(\varphi_{\mathrm{US} d}^{(2) b}\right)+\frac{1}{k} I_{\mathrm{US} d}^{(2) b},
$$

b.c. $\left\{\begin{array}{l}\varphi_{\mathrm{US} a}^{(2) b}=\mathscr{K}\left(\varphi_{\mathrm{US} a}^{(2) b}\right), \\ \varphi_{\mathrm{US} b}^{(2) b}=0, \\ \varphi_{\mathrm{US} c}^{(2) b}=0, \\ \varphi_{\mathrm{US} d}^{(2) b}=\mathscr{K}\left(\varphi_{\mathrm{US} d}^{(2) b}\right),\end{array} \quad \zeta_{r}>0, \quad r=1\right.$,

$$
\text { b.c. } \quad \varphi_{\mathrm{US} \alpha}^{(2) b} \rightarrow 0 \quad \text { as } r \rightarrow \infty \quad(\alpha=a, b, c, d),
$$




\section{Inversion of the transverse force on a spinning sphere}

where the operators $\mathscr{L}_{l}(\cdot), l=0,1,2$, are defined in Appendix B and the inhomogeneous terms $I_{\mathrm{UU} \alpha}^{(2)}, I_{\mathrm{SS} \alpha}^{(2)}, I_{\mathrm{US} \alpha}^{(2) \sharp}$ and $I_{\mathrm{US} \alpha}^{(2) b}(\alpha=a, b, c, d)$ are given by

$$
\left.\begin{array}{c}
I_{\mathrm{UU} a}^{(2)}=\mathscr{J}_{0}\left(\varphi_{\mathrm{U} a}^{(1)}, \varphi_{\mathrm{U} a}^{(1)}\right)-\frac{\zeta^{2}-\zeta_{r}^{2}}{2} \mathscr{J}_{2}\left(\varphi_{\mathrm{U} b}^{(1)}, \varphi_{\mathrm{U} b}^{(1)}\right)-\mathscr{J}_{3}\left(\varphi_{\mathrm{U} b}^{(1)}, \varphi_{\mathrm{U} b}^{(1)}\right), \\
I_{\mathrm{UU} b}^{(2)}=2 \mathscr{J}_{1}\left(\varphi_{\mathrm{U} a}^{(1)}, \varphi_{\mathrm{U} b}^{(1)}\right), \quad I_{\mathrm{UU} c}^{(2)}=\mathscr{J}_{2}\left(\varphi_{\mathrm{U} b}^{(1)}, \varphi_{\mathrm{U} b}^{(1)}\right), \\
I_{\mathrm{UU} d}^{(2)}=\frac{\zeta^{2}-\zeta_{r}^{2}}{2} \mathscr{J}_{2}\left(\varphi_{\mathrm{U} b}^{(1)}, \varphi_{\mathrm{U} b}^{(1)}\right)+\mathscr{J}_{3}\left(\varphi_{\mathrm{U} b}^{(1)}, \varphi_{\mathrm{U} b}^{(1)}\right), \\
I_{\mathrm{S} a}^{(2)}=-\frac{\zeta^{2}-\zeta_{r}^{2}}{2} \mathscr{J}_{2}\left(\varphi_{\mathrm{S}}^{(1)}, \varphi_{\mathrm{S}}^{(1)}\right)-\mathscr{J}_{3}\left(\varphi_{\mathrm{S}}^{(1)}, \varphi_{\mathrm{S}}^{(1)}\right), \quad I_{\mathrm{SS} b}^{(2)}=0, \\
I_{\mathrm{SS} c}^{(2)}=-\mathscr{J}_{2}\left(\varphi_{\mathrm{S}}^{(1)}, \varphi_{\mathrm{S}}^{(1)}\right), \\
I_{\mathrm{US} a}^{(2) \sharp}=2 \mathscr{J}_{1}\left(\varphi_{\mathrm{U} a}^{(1)}, \varphi_{\mathrm{S}}^{(1)}\right), \quad I_{\mathrm{US} b}^{(2) \sharp}=0, \quad I_{\mathrm{US} c}^{(2) \sharp}=2 \mathscr{J}_{2}\left(\varphi_{\mathrm{U} b}^{(1)}, \varphi_{\mathrm{S}}^{(1)}\right), \\
I_{\mathrm{US} d}^{(2) \sharp}=\left(\zeta^{2}-\zeta_{r}^{2}\right) \mathscr{J}_{2}\left(\varphi_{\mathrm{U} b}^{(1)}, \varphi_{\mathrm{S}}^{(1)}\right)+2 \mathscr{J}_{3}\left(\varphi_{\mathrm{U} b}^{(1)}, \varphi_{\mathrm{S}}^{(1)}\right), \\
I_{\mathrm{US} a}^{(2) b}=2 \zeta_{r} \mathscr{J}_{4}\left(\varphi_{\mathrm{U} b}^{(1)}, \varphi_{\mathrm{S}}^{(1)}\right), \quad I_{\mathrm{US} b}^{(2) b}=I_{\mathrm{US} c}^{(2) b}=I_{\mathrm{US} d}^{(2) b}=0 .
\end{array}\right\}
$$

It should be noted that $\mathscr{L}_{0}(\cdot), \mathscr{L}_{1}(\cdot)$ and $\mathscr{L}_{2}(\cdot)$ in $(\mathrm{E} 1),(\mathrm{E} 4),(\mathrm{E} 7)$ and $(\mathrm{E} 10)$ as well as $\mathscr{J}_{l}(\cdot, \cdot), l=0,1,2,3,4$, in (E13)-(E16) are functions of $r, \zeta_{r}$ and $\zeta$.

\section{Appendix F. Derivation of (4.7)}

In this appendix, we present a direct derivation of the formula (4.7). We begin with repeating the equation and boundary conditions for $\Phi_{\mathrm{U} *}^{(1)}$ and $\Phi_{\mathrm{US}}^{(2)}$. Writing $\Phi_{\mathrm{U} *}^{(1)}=\phi^{\mathrm{I}}$ and $\Phi_{\mathrm{US}}^{(2)}=\phi^{\mathrm{II}}$ for brevity, they are summarized as

(problem I)

$$
\begin{gathered}
\zeta_{i} \frac{\partial \phi^{\mathrm{I}}}{\partial x_{i}}=\frac{1}{k} \mathscr{L}\left(\phi^{\mathrm{I}}\right), \quad|\boldsymbol{x}|>1, \\
\phi^{\mathrm{I}}=\mathscr{K}\left(\phi^{\mathrm{I}}\right), \quad \zeta_{r}>0, \quad|\boldsymbol{x}|=1, \\
\phi^{\mathrm{I}} \rightarrow 2 \zeta_{3}, \quad \text { as }|\boldsymbol{x}| \rightarrow \infty ;
\end{gathered}
$$

(problem II)

$$
\begin{gathered}
\zeta_{i} \frac{\partial \phi^{\mathrm{II}}}{\partial x_{i}}=\frac{1}{k} \mathscr{L}\left(\phi^{\mathrm{II}}\right)+\frac{2}{k} \mathscr{J}\left(\Phi_{\mathrm{U}}^{(1)}, \Phi_{\mathrm{S}}^{(1)}\right), \quad|\boldsymbol{x}|>1, \\
\phi^{\mathrm{II}}=\mathscr{K}\left(\phi^{\mathrm{II}}\right)+2 \zeta_{\varphi} S \sin \theta \mathscr{K}\left(\Phi_{\mathrm{U}}^{(1)}\right), \quad \zeta_{r}>0, \quad|\boldsymbol{x}|=1, \\
\phi^{\mathrm{II}} \rightarrow 0, \quad \text { as }|\boldsymbol{x}| \rightarrow \infty,
\end{gathered}
$$

where $\Phi_{\mathrm{U}}^{(1)}$ and $\Phi_{\mathrm{S}}^{(1)}$ appearing in the inhomogeneous terms of problem II are the solutions to the problem (3.11)-(3.13) (with $J=\mathrm{U}$ or S). The following derivation follows the line of Takata (2009a) and takes several steps. 


\section{S. Taguchi and T. Tsuji}

We first define the reflection operator as follows: $F\left(\zeta_{i}\right)^{-} \equiv F\left(-\zeta_{i}\right)$ for any function $F$ of $\zeta_{i}$. Obviously, $\left(F^{-}\right)^{-}=F$ and $\left\langle F^{-}\right\rangle=\langle F\rangle$. Multiplying both sides of (F2a) by $\phi^{\mathrm{I}-}$ and integrating the result over $\zeta$, we obtain

$$
\frac{\partial}{\partial x_{i}}\left\langle\zeta_{i} \phi^{\mathrm{I}-} \phi^{\mathrm{II}}\right\rangle+\left\langle\phi^{\mathrm{II}-} \zeta_{i} \frac{\partial \phi^{\mathrm{I}}}{\partial x_{i}}\right\rangle-\frac{1}{k}\left\langle\phi^{\mathrm{I}-} \mathscr{L}\left(\phi^{\mathrm{II}}\right)\right\rangle=\frac{2}{k}\left\langle\phi^{\mathrm{I}-} \mathscr{J}\left(\Phi_{\mathrm{U}}^{(1)}, \Phi_{\mathrm{S}}^{(1)}\right)\right\rangle .
$$

Because the operator $\mathscr{L}$ is self-adjoint and commutes with the reflection operator $(\cdot)^{-}$, that is, $\mathscr{L}\left(F^{-}\right)=(\mathscr{L} F)^{-}$for any $F=F\left(\zeta_{i}\right)$, we can transform the third term as

$$
\begin{aligned}
\frac{1}{k}\left\langle\phi^{\mathrm{I}-} \mathscr{L}\left(\phi^{\mathrm{II}}\right)\right\rangle & =\frac{1}{k}\left\langle\phi^{\mathrm{II}} \mathscr{L}\left(\phi^{\mathrm{I}-}\right)\right\rangle=\frac{1}{k}\left\langle\phi^{\mathrm{II}} \mathscr{L}\left(\phi^{\mathrm{I}}\right)^{-}\right\rangle \\
& =\frac{1}{k}\left\langle\phi^{\mathrm{II}-} \mathscr{L}\left(\phi^{\mathrm{I}}\right)\right\rangle=\left\langle\phi^{\mathrm{II}-} \zeta_{i} \frac{\partial \phi^{\mathrm{I}}}{\partial x_{i}}\right\rangle=\text { (the second term) }
\end{aligned}
$$

Thus, we are left with the identity

$$
\frac{\partial}{\partial x_{i}}\left\langle\zeta_{i} \phi^{\mathrm{I}-} \phi^{\mathrm{II}}\right\rangle=\frac{2}{k}\left\langle\phi^{\mathrm{I}-} \mathscr{J}\left(\Phi_{\mathrm{U}}^{(1)}, \Phi_{\mathrm{S}}^{(1)}\right)\right\rangle .
$$

Further integration with respect to $x$ over the gas region enclosed by a sphere with radius $r_{0} \gg 1$ leads to, after letting $r_{0} \rightarrow \infty$,

$$
\begin{aligned}
& \lim _{r_{0} \rightarrow \infty} \int_{|x|=r_{0}}\left\langle\zeta_{r} \phi^{\mathrm{I}-} \phi^{\mathrm{II}}\right\rangle \mathrm{d} S-\int_{|x|=1}\left\langle\zeta_{r} \phi^{\mathrm{I}-} \phi^{\mathrm{II}}\right\rangle \mathrm{d} S \\
& \quad=\frac{2}{k} \int_{|x|>1}\left\langle\phi^{\mathrm{I}-} \mathscr{J}\left(\Phi_{\mathrm{U}}^{(1)}, \Phi_{\mathrm{S}}^{(1)}\right)\right\rangle \mathrm{d} \boldsymbol{x},
\end{aligned}
$$

where the divergence theorem has been used on the left-hand side. Note that the improper integral on the right-hand side is well defined (Proposition 3.1).

We argue that we can replace $\phi^{\mathrm{II}}$ in the second term of (F6) by $g_{w}^{\mathrm{II}} \equiv$ $2 \zeta_{\varphi} S \sin \theta \mathscr{K}\left(\left.\Phi_{\mathrm{U}}^{(1)}\right|_{r=1}\right)$. This is because

$$
\begin{aligned}
\left\langle\zeta_{r} \phi^{\mathrm{I}-}\left(\phi^{\mathrm{II}}-g_{w}^{\mathrm{II}}\right)\right\rangle & =\int_{\zeta_{r}>0} \zeta_{r} \phi^{\mathrm{I}-}\left(\phi^{\mathrm{II}}-g_{w}^{\mathrm{II}}\right) E \mathrm{~d} \zeta+\int_{\zeta_{r}<0} \zeta_{r} \phi^{\mathrm{I}-}\left(\phi^{\mathrm{II}}-g_{w}^{\mathrm{II}}\right) E \mathrm{~d} \zeta \\
& =\int_{\zeta_{r}>0} \zeta_{r} \phi^{\mathrm{I}-}\left(\phi^{\mathrm{II}}-g_{w}^{\mathrm{II}}\right) E \mathrm{~d} \zeta-\int_{\zeta_{r}>0} \zeta_{r} \phi^{\mathrm{I}}\left(\phi^{\mathrm{II}-}-g_{w}^{\mathrm{II}-}\right) E \mathrm{~d} \zeta \\
& =\mathscr{K}\left(\phi^{\mathrm{II}}\right) \int_{\zeta_{r}>0} \zeta_{r} \phi^{\mathrm{I}-} E \mathrm{~d} \zeta-\mathscr{K}\left(\phi^{\mathrm{I}}\right) \int_{\zeta_{r}>0} \zeta_{r}\left(\phi^{\mathrm{II}-}-g_{w}^{\mathrm{II}-}\right) E \mathrm{~d} \zeta \\
& =-\mathscr{K}\left(\phi^{\mathrm{II}}\right) \int_{\zeta_{r}<0} \zeta_{r} \phi^{\mathrm{I}} E \mathrm{~d} \zeta+\mathscr{K}\left(\phi^{\mathrm{I}}\right) \int_{\zeta_{r}<0} \zeta_{r} \phi^{\mathrm{II}} E \mathrm{~d} \zeta \\
& =\frac{1}{2 \sqrt{\pi}} \mathscr{K}\left(\phi^{\mathrm{II}}\right) \mathscr{K}\left(\phi^{\mathrm{I}}\right)-\frac{1}{2 \sqrt{\pi}} \mathscr{K}\left(\phi^{\mathrm{I}}\right) \mathscr{K}\left(\phi^{\mathrm{II}}\right) \\
& =0,
\end{aligned}
$$

holds on the boundary $|\boldsymbol{x}|=1$, where (F1b) and (F2b) have been used in the third equality. 


\section{Inversion of the transverse force on a spinning sphere}

Concerning the first term of (F6), we write the integral as

$$
\int_{|x|=r_{0}}\left\langle\zeta_{r} \phi^{\mathrm{II}} \phi^{\mathrm{I}-}\right\rangle \mathrm{d} S=\int_{|x|=r_{0}}\left\langle\zeta_{r} \phi^{\mathrm{II}}\left(\phi^{\mathrm{I}-}+2 \zeta_{3}\right)\right\rangle \mathrm{d} S-2 \int_{|x|=r_{0}}\left\langle\zeta_{3} \zeta_{r} \phi^{\mathrm{II}}\right\rangle \mathrm{d} S .
$$

Meanwhile, multiplying (F2a) by $\zeta_{3}$ and integrating over $\zeta$, we have $\partial\left\langle\zeta_{3} \zeta_{j} \phi^{\mathrm{II}}\right\rangle / \partial x_{j}=$ 0 for $|x|>1$, implying that $\int_{|x|=r_{0}}\left\langle\zeta_{3} \zeta_{r} \phi^{\mathrm{II}}\right\rangle \mathrm{d} S=\int_{|x|=1}\left\langle\zeta_{3} \zeta_{r} \phi^{\mathrm{II}}\right\rangle \mathrm{d} S$. Therefore, we can transform the last term of (F8) as

$$
\begin{aligned}
-2 \int_{|x|=r_{0}}\left\langle\zeta_{3} \zeta_{r} \phi^{\mathrm{II}}\right\rangle \mathrm{d} S & =-2 \int_{|x|=1}\left\langle\zeta_{3} \zeta_{r} \phi^{\mathrm{II}}\right\rangle \mathrm{d} S \\
& =-2 \int_{|x|=1}\left\langle\zeta_{3} \zeta_{r} \Phi_{\mathrm{US}}^{(2)}\right\rangle \mathrm{d} S=-S V h_{L} .
\end{aligned}
$$

Here, the last equality follows from (3.81)-(3.83) and (3.86) together with (3.21) (note that $\left.\int_{|x|=1}\left\langle\zeta_{3} \zeta_{r} \Phi_{\mathrm{US}}^{(2) b}\right\rangle \mathrm{d} S=0\right)$. Concerning the first term of (F8), it can be shown that $\phi^{\mathrm{II}}$ $\left(=\Phi_{\mathrm{US}}^{(2)}\right)$ behaves for large $|x|$ as

$$
\phi^{\mathrm{II}}(\boldsymbol{x}, \zeta)=2 \zeta_{i} d_{i}^{\mathrm{II}}+O\left(|x|^{-2}\right), \quad|x| \gg 1,
$$

where $d_{i}^{\text {II }}$ is a quantity of $O\left(|x|^{-1}\right.$ ) (see the last paragraph of this appendix). Since $\phi^{\mathrm{I}}$ behaves as $\phi^{\mathrm{I}}(\boldsymbol{x}, \zeta)=2 \zeta_{3}+2 \zeta_{i} d_{i}^{\mathrm{I}}+O\left(|\boldsymbol{x}|^{-2}\right)$ for $|\boldsymbol{x}| \gg 1$, where $d_{i}^{\mathrm{I}}$ is a quantity of $O\left(|x|^{-1}\right.$ ) (Proposition 3.1), we conclude that the first term on the right-hand side of (F8) vanishes as $r_{0} \rightarrow \infty$. Then, the identity (4.7) follows from (F6), since $\phi^{\mathrm{I}}=\Phi_{\mathrm{U} *}^{(1)}$, $g_{w}^{\mathrm{II}}=I_{w, \mathrm{US}}^{(2)}$ and $I_{\mathrm{US}}^{(2)}=2 \mathscr{J}\left(\Phi_{\mathrm{U}}^{(1)}, \Phi_{\mathrm{S}}^{(1)}\right)$.

Finally, we briefly explain the derivation of the asymptotic property given in (F10). To see the asymptotic behaviour of $\Phi_{\text {US }}^{(2)}$ at large $|x| \gg 1$, we apply a procedure similar to that described in the proof of Proposition 3.1. A difference is that the equation for $\Phi_{\mathrm{US}}^{(2)}$ has an inhomogeneous term, which depends on $\Phi_{\mathrm{U}}^{(1)}$ and $\Phi_{\mathrm{S}}^{(1)}$. In this case, we can simplify the analysis by using the S expansion (Sone 2007) instead of applying the Grad-Hilbert expansion to $(\mathrm{F} 2 a)$. More precisely, we first apply the $\mathrm{S}$ expansion to (2.3), assuming the local Knudsen number is small, to derive the Navier-Stokes-type system equivalent to (3.36)-(3.38), which we sum up to obtain the equations for the (total) macroscopic variables for $\phi_{F}$ in the far region. Then, substituting the expansion (3.2) and rearranging the terms, we extract a system of partial differential equations describing the macroscopic behaviour corresponding to $\phi_{F 2}$. The derived equations consist of the Stokes equations for $u_{i F 2}$ and $P_{F 2}$ with sources and the Laplace equation for $\tau_{F 2}$ with sources, whose homogeneous counterparts are similar to (D1) (the source terms consist of lower-order terms, i.e. $u_{i F 1}, \tau_{F 1}$, etc.). The remaining process is parallel to that in Proposition 3.1 for the derivation of (D4) and that of (3.47)-(3.49). The asymptotic property (F10) is obtained in this way.

\section{Appendix G. Asymptotic analysis in the case of a free molecular gas}

In this appendix, we give a summary of the asymptotic analysis $(\S 3)$ in the case of a free molecular (or collisionless) gas. We note that Wang (1972), Ivanov \& Yanshin (1980) and Borg et al. (2003) investigated this case and showed the essential feature of the force and the torque acting on a sphere moving in a collisionless gas. Here, we study the same 


\section{S. Taguchi and T. Tsuji}

problem in the framework of the present asymptotic analysis. We use the notation of the main text. Then, the basic equation is given by (2.3) with the right-hand side replaced by zero. The boundary conditions (2.5) and (2.8) are unchanged.

\section{G.1. Leading order in $\varepsilon$}

The leading-order solution is obtained in the form of (3.10) with (3.16) with $\varphi_{\mathrm{U} a}^{(1)}, \varphi_{\mathrm{U} b}^{(1)}$ and $\varphi_{\mathrm{S}}^{(1)}$ given by

$$
\begin{gathered}
\varphi_{\mathrm{U} a}^{(1)}= \begin{cases}-\sqrt{\pi} Z_{1}\left(r, \theta_{\zeta}\right), & 0 \leq \theta_{\zeta}<\sin ^{-1}\left(\frac{1}{r}\right), \\
2 \zeta \cos \theta_{\zeta}, & \sin ^{-1}\left(\frac{1}{r}\right)<\theta_{\zeta} \leq \pi,\end{cases} \\
\varphi_{\mathrm{U} b}^{(1)}= \begin{cases}-\frac{\sqrt{\pi}}{\zeta} Z_{2}\left(r, \theta_{\zeta}\right), & 0 \leq \theta_{\zeta}<\sin ^{-1}\left(\frac{1}{r}\right), \\
-2, & \sin ^{-1}\left(\frac{1}{r}\right)<\theta_{\zeta} \leq \pi,\end{cases} \\
\varphi_{\mathrm{S}}^{(1)}= \begin{cases}2 r, & 0 \leq \theta_{\zeta}<\sin ^{-1}\left(\frac{1}{r}\right), \\
0, & \sin ^{-1}\left(\frac{1}{r}\right)<\theta_{\zeta} \leq \pi,\end{cases}
\end{gathered}
$$

where $\theta_{\zeta}=\cos ^{-1}\left(\zeta_{r} / \zeta\right)$ and

$$
\begin{gathered}
Z_{1}\left(r, \theta_{\zeta}\right)=\sqrt{1-r^{2} \sin ^{2} \theta_{\zeta}} \cos \theta_{\zeta}+r \sin ^{2} \theta_{\zeta} \\
Z_{2}\left(r, \theta_{\zeta}\right)=r \cos \theta_{\zeta}-\sqrt{1-r^{2} \sin ^{2} \theta_{\zeta}}
\end{gathered}
$$

$\left(0 \leq \theta_{\zeta} \leq \sin ^{-1}(1 / r)\right)$. Note that $\varphi_{\mathrm{U} a}^{(1)}, \varphi_{\mathrm{U} b}^{(1)}$ and $\varphi_{\mathrm{S}}^{(1)}$ are discontinuous at $\theta_{\zeta}=\sin ^{-1}(1 / r)$

The leading-order macroscopic quantities are given in the form of (3.22). In particular,

$$
\begin{gathered}
\tilde{u}_{r, \mathrm{U} a}^{(1)}=\frac{1}{8}\left(1-\frac{1}{r}\right)\left(3+\frac{3}{r}+\frac{2}{r^{2}}\right)+\frac{1}{2}\left(1-\frac{1}{r^{2}}\right)^{3 / 2} \\
+\frac{r}{16}\left(1-\frac{1}{r^{2}}\right)^{2} \ln \left(\frac{r+1}{r-1}\right) \\
\tilde{u}_{t, \mathrm{U} b}^{(1)}=-\frac{5}{16}-\frac{1}{16 r^{2}}-\frac{1}{8 r^{3}}-\frac{1}{4} \sqrt{1-\frac{1}{r^{2}}\left(\frac{1}{r^{2}}+2\right)} \\
-\frac{r}{32}\left(1-\frac{1}{r^{2}}\right)\left(\frac{1}{r^{2}}+3\right) \ln \left(\frac{r+1}{r-1}\right) \\
\tilde{u}_{t, \mathrm{~S}}^{(1)}=\frac{r}{2}\left[1-\sqrt{1-\frac{1}{r^{2}}}\left(1+\frac{1}{2 r^{2}}\right)\right] \\
\tilde{P}_{r r, \mathrm{U} a}^{(1)}=-\frac{\sqrt{\pi}}{10}\left[2 r-\left(1-\frac{1}{r^{2}}\right)^{3 / 2}\left(2 r+\frac{3}{r}\right)+\frac{20}{\pi r^{2}}\left(2-\frac{1}{r^{2}}\right)+\frac{1}{r^{2}}\left(5-\frac{2}{r^{2}}\right)\right]
\end{gathered}
$$


Inversion of the transverse force on a spinning sphere

$$
\begin{gathered}
\tilde{P}_{r t, \mathrm{U} b}^{(1)}=-\frac{\sqrt{\pi}}{10}\left[r-\left(1-\frac{1}{r^{2}}\right)^{3 / 2}\left(r+\frac{3}{2 r}\right)-\left(\frac{10}{\pi}+1\right) \frac{1}{r^{4}}\right], \\
\tilde{P}_{r t, \mathrm{~S}}^{(1)}=\frac{1}{\sqrt{\pi} r^{3}} .
\end{gathered}
$$

With (G3d)-(G3f), we can derive $P_{r r F 1}, P_{r \theta F 1}$ and $P_{r \varphi F 1}$ from (3.22g)-(3.22i).

\section{G.2. Second order in $\varepsilon$}

Next, we consider the second-order problem in $\varepsilon$. The equation is $\zeta_{i} \partial \phi_{F 2} / \partial x_{i}=$ 0 , equipped with the boundary condition on the sphere (3.65) with $\mathscr{K}\left(\phi_{F 1}\right)=$ $-\sqrt{\pi}(U \cos \theta+V \sin \theta \cos \varphi)$. The condition at infinity is given by

$$
\phi_{F 2} \rightarrow 2\left(e_{j} \zeta_{j}\right)^{2}-1 \quad \text { as }|x| \rightarrow \infty
$$

where $e_{i}$ is defined in (3.30a). Note that the condition (G4) is identical to the condition (3.66) with $c_{1}=0$. This is consistent with the fact that $c_{1}$ vanishes as $k \rightarrow \infty$ (see (3.50a)). Accordingly, we can obtain the solution in the form $\phi_{F 2}=\Phi_{\mathrm{UU}}^{(2)}+\Phi_{\mathrm{SS}}^{(2)}+\Phi_{\mathrm{US}}^{(2)}$ (cf. (3.69) with $c_{1}=0$ ), where $\Phi_{\mathrm{UU}}^{(2)}, \Phi_{\mathrm{SS}}^{(2)}$ and $\Phi_{\mathrm{US}}^{(2)}$ are of the form (3.79)-(3.83). Moreover, because inhomogeneous terms are absent in the problem for $\Phi_{\mathrm{US}}^{(2) b}$ (see (E16)), $\Phi_{\mathrm{US}}^{(2) b}$ is identically zero for the free molecular gas (thus, $\Phi_{\mathrm{US}}^{(2)}=\Phi_{\mathrm{US}}^{(2) \sharp}$ ). Then $\varphi_{\mathrm{UU} \alpha}^{(2)}, \varphi_{\mathrm{SS} \alpha}^{(2)}$ and $\varphi_{\mathrm{US} \alpha}^{(2) \sharp}(\alpha=a, b, c, d)$ are given by

$$
\begin{gathered}
\varphi_{\mathrm{UU} a}^{(2)}=Z_{1}^{2}-\frac{Z_{2}^{2}}{2} \sin ^{2} \theta_{\zeta}, \quad \varphi_{\mathrm{UU} b}^{(2)}=2 \frac{Z_{1} Z_{2}}{\zeta}, \quad \varphi_{\mathrm{UU} c}^{(2)}=\frac{Z_{2}^{2}}{\zeta^{2}}, \\
\varphi_{\mathrm{UU} d}^{(2)}=\frac{Z_{2}^{2}}{2} \sin ^{2} \theta_{\zeta}, \quad \varphi_{\mathrm{SS} a}^{(2)}=Z_{1}^{2}-\left(\frac{Z_{2}^{2}}{\zeta^{2}}+2 r^{2}\right) \frac{\zeta^{2} \sin ^{2} \theta_{\zeta}}{2}, \\
\varphi_{\mathrm{SS} b}^{(2)}=2 \frac{Z_{1} Z_{2}}{\zeta}, \quad \varphi_{\mathrm{SS} c}^{(2)}=\frac{Z_{2}^{2}}{\zeta^{2}}-2 r^{2}, \\
\varphi_{\mathrm{SS} d}^{(2)}=\left(\frac{Z_{2}^{2}}{\zeta^{2}}+2 r^{2}\right) \frac{\zeta^{2} \sin ^{2} \theta_{\zeta}}{2}-1, \\
\varphi_{\mathrm{US} a}^{(2) \sharp}=-2 \sqrt{\pi} r Z_{1}, \quad \varphi_{\mathrm{US} b}^{(2) \sharp}=0, \quad \varphi_{\mathrm{US} c}^{(2) \sharp}=-2 \sqrt{\pi} r \frac{Z_{2}}{\zeta}, \\
\varphi_{\mathrm{US} d}^{(2) \sharp}=-2 \sqrt{\pi} r \frac{Z_{2}}{\zeta} \frac{\zeta^{2} \sin ^{2} \theta_{\zeta}}{2},
\end{gathered}
$$

for $0 \leq \theta_{\zeta}<\sin ^{-1}(1 / r)$ and

$$
\left.\begin{array}{c}
\varphi_{\mathrm{UU} a}^{(2)}=\zeta^{2}\left(3 \cos ^{2} \theta_{\zeta}-1\right), \quad \varphi_{\mathrm{UU} b}^{(2)}=-4 \zeta \cos \theta_{\zeta}, \quad \varphi_{\mathrm{UU} c}^{(2)}=2, \\
\varphi_{\mathrm{UU} d}^{(2)}=\zeta^{2} \sin ^{2} \theta_{\zeta}-1, \quad \varphi_{\mathrm{SS} a}^{(2)}=0, \quad \varphi_{\mathrm{SS} b}^{(2)}=0, \quad \varphi_{\mathrm{SS} c}^{(2)}=0, \quad \varphi_{\mathrm{SS} d}^{(2)}=0, \\
\varphi_{\mathrm{US} a}^{(2) \sharp}=0, \quad \varphi_{\mathrm{US} b}^{(2) \sharp}=0, \quad \varphi_{\mathrm{US} c}^{(2) \sharp}=0, \quad \varphi_{\mathrm{US} d}^{(2) \sharp}=0,
\end{array}\right\}
$$

for $\sin ^{-1}(1 / r)<\theta_{\zeta} \leq \pi$. Here, $Z_{1}=Z_{1}\left(r, \theta_{\zeta}\right)$ and $Z_{2}=Z_{2}\left(r, \theta_{\zeta}\right)$ are defined in (G2). Note that the solutions are discontinuous at $\theta_{\zeta}=\sin ^{-1}(1 / r)$. 


\section{S. Taguchi and T. Tsuji}

Unlike the case with finite Knudsen numbers, we do not require the slowly varying outer solution, and the inner solution is directly connected to the boundary condition at infinity in the case of a free molecular gas.

The components $\left(P_{r r F 2}, P_{r \theta F 2}, P_{r \varphi F 2}\right)$ of the second-order stress tensor $P_{i j F 2}$ are given in the from $(3.84 a)-(3.84 c)$ with $c_{1}=0$, where

$$
\begin{aligned}
& \tilde{P}_{r r}\left[\varphi_{\mathrm{UU} a}^{(2)}\right]=-\frac{1}{140}\left[-\left(1-\frac{1}{r}\right)\left(1+\frac{1}{r}\right)\left(2-\frac{9}{r^{2}}\right)\left(3-\frac{38}{r^{2}}\right) r^{2}\left(1-\sqrt{1-\frac{1}{r^{2}}}\right)\right. \\
& \left.-277+\frac{445}{r^{2}}-\frac{84}{r^{3}}-\frac{342}{r^{4}}+\frac{48}{r^{5}}\right], \\
& \tilde{P}_{r r}\left[\varphi_{\mathrm{UU} d}^{(2)}\right]=\frac{1}{140}\left[-\left(1-\frac{1}{r}\right)\left(1+\frac{1}{r}\right)\left(2-\frac{11}{r^{2}}+\frac{114}{r^{4}}\right) r^{2}\left(1-\sqrt{1-\frac{1}{r^{2}}}\right)\right. \\
& \left.+\left(1-\frac{1}{r}\right)\left(1+\frac{1}{r}+\frac{126}{r^{2}}+\frac{98}{r^{3}}-\frac{16}{r^{4}}\right)\right], \\
& \tilde{P}_{r r}\left[\varphi_{\mathrm{SS} a}^{(2)}\right]=\frac{1}{140}\left[-\left(1-\frac{1}{r}\right)\left(1+\frac{1}{r}\right)\left(64+\frac{68}{r^{2}}-\frac{27}{r^{4}}\right) r^{2}\left(1-\sqrt{1-\frac{1}{r^{2}}}\right)\right. \\
& \left.+\left(1-\frac{1}{r}\right)\left(32+\frac{32}{r}-\frac{63}{r^{2}}+\frac{21}{r^{3}}+\frac{48}{r^{4}}\right)\right] \text {, } \\
& \tilde{P}_{r r}\left[\varphi_{\mathrm{SS} d}^{(2)}\right]=-\frac{1}{140}\left[-\left(1-\frac{1}{r}\right)\left(1+\frac{1}{r}\right)\left(68+\frac{46}{r^{2}}-\frac{9}{r^{4}}\right) r^{2}\left(1-\sqrt{1-\frac{1}{r^{2}}}\right)\right. \\
& \left.+\left(1-\frac{1}{r}\right)\left(34+\frac{34}{r}-\frac{21}{r^{2}}+\frac{7}{r^{3}}+\frac{16}{r^{4}}\right)\right] \\
& \tilde{P}_{r r}\left[\varphi_{\mathrm{USd}}^{(2) \sharp}\right]=\frac{1}{12}\left\{3\left(1-\frac{1}{r}\right)^{2}\left(1+\frac{1}{r}\right)^{2}\left(1+\frac{1}{r^{2}}\right) r\left[\frac{r}{2} \ln \left(\frac{r-1}{r+1}\right)+1\right]\right. \\
& \left.+\left(1-\frac{1}{r}\right) \frac{1}{r}\left(1-\frac{11}{r}-\frac{5}{r^{2}}+\frac{3}{r^{3}}\right)\right\} \text {, } \\
& \tilde{P}_{r r}\left[\varphi_{\mathrm{US} a}^{(2) b}\right]=\tilde{P}_{r r}\left[\varphi_{\mathrm{US} d}^{(2) b}\right]=0, \\
& \tilde{P}_{r t}\left[\varphi_{\mathrm{UU} b}^{(2)}\right]=-\frac{1}{35}\left[\left(1-\frac{1}{r}\right)\left(1+\frac{1}{r}\right)\left(8+\frac{40}{r^{2}}+\frac{57}{r^{4}}\right) r^{2}\left(\sqrt{1-\frac{1}{r^{2}}}-1\right)\right. \\
& \left.+74+\frac{17}{r^{2}}-\frac{7}{r^{3}}-\frac{57}{r^{4}}+\frac{8}{r^{5}}\right] \\
& \tilde{P}_{r t}\left[\varphi_{\mathrm{SS} b}^{(2)}\right]=-\frac{1}{70}\left[\left(1-\frac{1}{r}\right)\left(1+\frac{1}{r}\right)\left(16+\frac{10}{r^{2}}+\frac{9}{r^{4}}\right) r^{2}\left(\sqrt{1-\frac{1}{r^{2}}}-1\right)\right.
\end{aligned}
$$


Inversion of the transverse force on a spinning sphere

$$
\begin{aligned}
\left.+\left(1-\frac{1}{r}\right)^{2}\left(8+\frac{16}{r}+\frac{23}{r^{2}}+\frac{16}{r^{3}}\right)\right], \\
\tilde{P}_{r t}\left[\varphi_{\mathrm{US} b}^{(2) \sharp}\right]=0, \\
\tilde{P}_{r t}\left[\varphi_{\mathrm{US} b}^{(2) b}\right]=0, \\
\tilde{P}_{r t}\left[\varphi_{\mathrm{US} a}^{(2) \sharp}\right]=-\frac{1}{12}\left\{3\left(1-\frac{1}{r}\right)^{2}\left(1+\frac{1}{r}\right)^{2}\left(1+\frac{1}{r^{2}}\right) r\left[\frac{r}{2} \ln \left(\frac{r-1}{r+1}\right)+1\right]\right. \\
\left.+\left(1+\frac{6}{r^{2}}+\frac{8}{r^{3}}-\frac{3}{r^{4}}\right) \frac{1}{r}\right\} .
\end{aligned}
$$

\section{Appendix H. Some comments on the numerical computations}

In this appendix, we give more details of the numerical computations for $h_{L}$. We restrict the ranges of $r$ and $\zeta$ to $1 \leq r \leq r_{\max }$ and $0 \leq \zeta \leq \zeta_{\max }$, where $r_{\max }$ and $\zeta_{\max }$ are appropriately chosen constants, which are large enough. In our computations, the value of $r_{\text {max }}$ is varied depending on $k$ ( $r_{\max }=150 k$ is used for most of the cases). For numerical integration, the domain $\left[1, r_{\max }\right] \times[0, \pi] \times\left[0, \zeta_{\max }\right]\left(r \times \theta_{\zeta} \times \zeta\right.$ directions $)$ is further subdivided by $\left(N_{r}+1\right) \times\left(2 N_{\theta_{\zeta}}+1\right) \times\left(N_{\zeta}+1\right)$ lattice points non-uniformly distributed along each direction. For every $k$, the same lattice system is used for solving both problems $\mathrm{U}$ and $\mathrm{S}$. Note that $\left(\varphi_{\mathrm{U} a}^{(1)}, \varphi_{\mathrm{U} b}^{(1)}\right)$ and $\varphi_{\mathrm{S}}^{(1)}$ are discontinuous on $\theta_{\zeta}=\sin ^{-1}(1 / r)$ and this discontinuity is fully accounted for in the present computations (see Takata et al. 1993; Taguchi \& Suzuki 2017). The values of $N_{r}, N_{\theta_{\zeta}}$ and $N_{\zeta}$ used for the results shown in table 5 and figure 3 are as follows:

$$
\left(N_{r}, N_{\theta_{\zeta}}, N_{\zeta}\right)= \begin{cases}(3200,1024,74) & \text { for } 0.01 \leq k \leq 0.09 \\ (1600,512,74) & \text { for } 0.09 \leq k \leq 50\end{cases}
$$

Note that, by restricting the range of $r$ to $1 \leq r \leq r_{\max }$, the upper limits of the integrals $I_{1}$ and $I_{2}$ in (4.8) are also restricted. Using the asymptotic expressions given in Proposition 3.1, we can estimate the error introduced by this restriction as follows. That is, writing $\int_{0}^{\infty}=\int_{0}^{r_{\max }}+\int_{r_{\max }}^{\infty}$, the contribution from the second integral is found to be $2 k c_{1} c_{4} / r_{\max }+O\left(r_{\max }^{-2}\right)$ for $I_{1}+I_{2}$. Since $c_{1}$ and $c_{4}$ are known from $h_{D}$ and $h_{M}$ (see Proposition 3.2), the term $2 k c_{1} c_{4} / r_{\max }$ is known and can be used to correct the numerical result for the first integral. The results presented in table 5 and figure 3 are obtained in this way.

To assess the accuracy of the data presented in table 5, we consider the following quantity:

$$
\mathcal{E}=\left|\frac{h_{L}\left[S_{1}\right]-h_{L}\left[S_{1 / 2}\right]}{h_{L}\left[S_{1}\right]}\right| .
$$

Here, $h_{L}\left[S_{1}\right]$ denotes the value obtained using a lattice system with $\left(N_{r}, N_{\theta_{\zeta}}, N_{\zeta}\right)$ shown in (H1) and $h_{L}\left[S_{1 / 2}\right]$ the value obtained using a lattice system with $N_{r}$ and $N_{\theta_{\zeta}}$ halved. 


\section{S. Taguchi and T. Tsuji}

Then, the values of $\mathcal{E}$ in our computations are summarized as

$$
\mathcal{E} \leq \begin{cases}8.7 \times 10^{-6} & (0.01 \leq k \leq 0.04) \\ 2.8 \times 10^{-5} & (0.05 \leq k \leq 0.09) \\ 7.3 \times 10^{-4} & (0.1 \leq k \leq 0.4) \\ 6.4 \times 10^{-3} & (0.5 \leq k \leq 0.9) \\ 1.2 \times 10^{-4} & (1 \leq k \leq 4) \\ 5.6 \times 10^{-5} & (5 \leq k \leq 9) \\ 1.5 \times 10^{-5} & (10 \leq k \leq 20) \\ 2.6 \times 10^{-4} & (k=50)\end{cases}
$$

\section{REFERENCES}

AndReEV, A.P. \& Popov, V.N. 2010 Analytic solution of the problem of rotation of a sphere in a rarefied molecular gas with allowance for the accommodation coefficients. Fluid Dyn. 45, 493-505.

Andries, P., Le Tallec, P., Perlat, J.-P. \& Perthame, B. 2000 The Gaussian-BGK model of Boltzmann equation with small Prandtl number. Eur. J. Mech. (B/Fluids) 19 (6), 813-830.

AOKI, K. \& Sone, Y. 1987 Temperature field induced around a sphere in a uniform flow of a rarefied gas. Phys. Fluids 30 (7), 2286-2288.

Beresnev, S.A., Chernyak, V.G. \& Fomyagin, G.A. 1990 Motion of a spherical particle in a rarefied gas. Part 2. Drag and thermal polarization. J. Fluid Mech. 219, 405-421.

Bhatnagar, P.L., Gross, E.P. \& KroOK, M. 1954 A model for collision processes in gases. I. Small amplitude processes in charged and neutral one-component systems. Phys. Rev. 94, 511-525.

Borg, K.I., SöDerholm, L.H. \& EsSÉn, H. 2003 Force on a spinning sphere moving in a rarefied gas. Phys. Fluids 15 (3), 736-741.

BRULl, S. \& SCHNEIDER, J. 2008 A new approach for the ellipsoidal statistical model. Contin. Mech. Thermodyn. 20 (2), 63-74.

Cercignani, C. 1988 The Boltzmann Equation and its Applications. Springer-Verlag.

Cercignani, C., Pagani, C.D. \& Bassanini, P. 1968 Flow of a rarefied gas past an axisymmetric body. II. Case of a sphere. Phys. Fluids 11 (7), 1399-1403.

Chester, W., Breach, D.R. \& Proudman, I. 1969 On the flow past a sphere at low Reynolds number. J. Fluid Mech. 37, 751-760.

Epstein, P.S. 1924 On the resistance experienced by spheres in their motion through gases. Phys. Rev. 23, 710-733.

Holway, J. 1966 New statistical models for kinetic theory: methods of construction. Phys. Fluids 9 (9), $1658-1673$.

IVANOV, S.G. \& YANSHIN, A.M. 1980 Forces and moments acting on bodies rotating about a symmetry axis in a free molecular flow. Fluid Dyn. 15 (3), 449-453.

Kalempa, D. \& Sharipov, F. 2020 Drag and thermophoresis on a sphere in a rarefied gas based on the Cercignani-Lampis model of gas-surface interaction. J. Fluid Mech. 900, A37.

Knudsen, M. \& Weber, S. 1911 Luftwiderstand gegen die langsame Bewegung kleiner Kugeln. Ann. Phys. 341 (15), 981-994.

Kogan, M.N. 1969 Rarefied Gas Dynamics. Springer Science+Business Media.

LAW, W.S. \& LOYALKA, S.K. 1986 Motion of a sphere in a rarefied gas. II. Role of temperature variation in the Knudsen layer. Phys. Fluids 29 (11), 3886-3888.

LiU, N. \& BOGY, D.B. 2008 Forces on a rotating particle in a shear flow of a highly rarefied gas. Phys. Fluids 20 (10), 107102.

LOYALKA, S.K. 1992 Motion of a sphere in a gas: numerical solution of the linearized Boltzmann equation. Phys. Fluids A 4 (5), 1049-1056.

Proudman, I. \& Pearson, J.R.A. 1957 Expansions at small Reynolds numbers for the flow past a sphere and a circular cylinder. J. Fluid Mech. 2, 237-262.

Rubinow, S.I. \& KeLleR, J.B. 1961 The transverse force on a spinning sphere moving in a viscous fluid. J. Fluid Mech. 11, 447-459. 


\section{Inversion of the transverse force on a spinning sphere}

SHARIPOV, F. 1994 Onsager-Casimir reciprocity relations for open gaseous systems at arbitrary rarefaction I. General theory for single gas. Physica A 203, 437-456.

ShARIPOV, F. 2011 Power-series expansion of the Boltzmann equation and reciprocal relations for nonlinear irreversible phenomena. Phys. Rev. E 84, 061137.

SonE, Y. 1971 Asymptotic theory of flow of rarefied gas over a smooth boundary II. In Rarefied Gas Dynamics (ed. D. Dini), vol. 2, pp. 737-749. Editrice Tecnico Scientfica.

Sone, Y. 2002 Kinetic Theory and Fluid Dynamics. Birkhäuser. Supplementary Notes and Errata: Kyoto University Research Information Repository (http://hdl.handle.net/2433/66099).

Sone, Y. 2007 Molecular Gas Dynamics: Theory, Techniques, and Applications. Birkhäuser. Supplementary Notes and Errata: Kyoto University Research Information Repository (http://hdl.handle.net/2433/66098).

SONE, Y. \& AOKI, K. 1977 a Forces on a spherical particle in a slightly rarefied gas. In Rarefied Gas Dynamics (ed. J.L. Potter), pp. 417-433. AIAA.

Sone, Y. \& AOKI, K. 1977 b Slightly rarefied gas flow over a specularly reflecting body. Phys. Fluids 20 (4), 571-576.

TAGUCHI, S. 2015 Asymptotic theory of a uniform flow of a rarefied gas past a sphere at low Mach numbers. J. Fluid Mech. 774, 363-394.

TAGUChi, S., SAITO, K. \& TAKATA, S. 2019 A rarefied gas flow around a rotating sphere: diverging profiles of gradients of macroscopic quantities. J. Fluid Mech. 862, 5-33.

TAGUCHI, S. \& SuzUKI, T. 2017 Asymptotic far-field behavior of macroscopic quantities in a problem of slow uniform rarefied gas flow past a sphere. Phys. Rev. Fluids 2, 113401.

TAguchi, S., Tsuji, T. \& Kotera, M. 2021 Transient behaviour of a rarefied gas around a sphere caused by impulsive rotation. J. Fluid Mech. 909, A6.

TAKATA, S. 2009a Symmetry of the linearized Boltzmann equation and its application. J. Stat. Phys. 136 (4), 751-784.

TAKATA, S. 2009b Symmetry of the linearized Boltzmann equation II: entropy production and Onsager-Casimir relation. J. Stat. Phys. 136 (5), 945-983.

TAKATA, S., SONE, Y. \& AOKI, K. 1993 Numerical analysis of a uniform flow of a rarefied gas past a sphere on the basis of the Boltzmann equation for hard-sphere molecules. Phys. Fluids A 5 (3), 716-737.

VAN DYKe, M. 1975 Perturbation Methods in Fluid Mechanics. Parabolic Press.

VOLKOV, A.N. 2011 Transitional flow of a rarefied gas over a spinning sphere. J. Fluid Mech. 683, 320-345.

WANG, C.-T. 1972 Free molecular flow over a rotating sphere. AIAA J. 10 (5), 713-714.

WELANDER, P. 1954 On the temperature jump in a rarefied gas. Ark. Fys. 7, 507-553.

Willis, D.R. 1966 Sphere drag at high Knudsen number and low Mach number. Phys. Fluids 9 (12), $2522-2524$. 